\title{
FINGERPRINT ENHANCEMENT BY DIRECTIONAL FILTERING
}

by

\author{
SREYA CHAKRABORTY \\ Presented to the Faculty of the Graduate School of \\ The University of Texas at Arlington in Partial Fulfillment \\ of the Requirements \\ for the Degree of \\ MASTER OF SCIENCE IN ELECTRICAL ENGINEERING
}

THE UNIVERSITY OF TEXAS AT ARLINGTON

DECEMBER 2011 


\section{ACKNOWLEDGEMENTS}

It is a pleasure to thank many people who made this thesis possible.

This work would not have been possible without the support from Prof. Dr. K.R.Rao whose guidance, I chose this topic. I would like to gratefully acknowledge the supervision of my advisor Dr. K.R.Rao who has been abundantly helpful and has assisted me in numerous ways. I specially thank him for his infinite patience. The discussions I have had with him are invaluable.

I would like to thank Dr. A. Davis and Dr. M. Manry for being a part of my thesis committee. I would also like to extend my gratitude to A.M.Raičević and B.M. Popović.

My final words go to my family. I want to thank my family, whose love and guidance is with me in whatever I pursue.

On a different note, many people have been a part of my graduate education and I am highly grateful to all of them.

November 7, 2011 


\author{
ABSTRACT \\ FINGERPRINT ENHANCEMENT \\ BY DIRECTIONAL \\ FILTERING \\ Sreya Chakraborty, MS
}

The University of Texas at Arlington, 2011

Supervising Professor: Kamisetty Rao

Although inkless methods for taking fingerprint impressions are now available, these methods still suffer from the positional shifting caused by the skin elasticity. The non cooperative attitude of suspects or criminals also leads to smearing in parts of the fingerprint impressions. Thus a substantial amount of research reported in the literature on fingerprint identification is devoted to image enhancement techniques.

The important step in fingerprint matching is the reliable fingerprint recognition. Automatic Fingerprint Recognition System relies on the input fingerprint for feature extraction. Hence, the effectiveness of feature extraction relies heavily on the quality of input fingerprint images. In this thesis adaptive filtering in frequency domain in order to enhance fingerprint image is proposed.

Several stages of processing take place when an Automated Fingerprint Identification System (AFIS) is used to match an unknown fingerprint [2].

1) The fingerprint is first enhanced to remove noisy and any irrelevant information. 
2) The enhanced image is then encoded into a form suitable for comparison with the records held in the database. The encoded data consists of various key information of the fingerprint image like its minutiae.

3) Matching is then performed by comparing the encoded record against those held in the database.

4) Verification stage is performed wherein a fingerprint expert visually compares the unknown print with the candidates' fingerprints.

In this thesis Gabor filter is used for fingerprint enhancement technique. Because of its frequency selective and orientation selective properties it proves to be useful for fingerprint enhancement. The primary advantage of the approach is improved translation and rotation invariance. 


\section{TABLE OF CONTENTS}

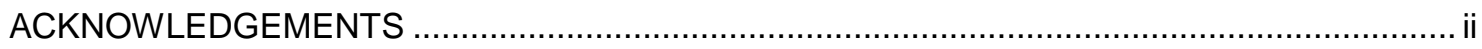

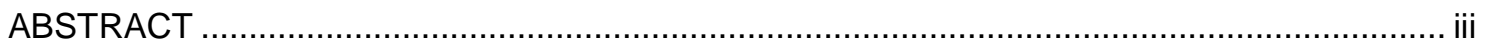

LIST OF ILLUSTRATIONS.................................................................................................... viii

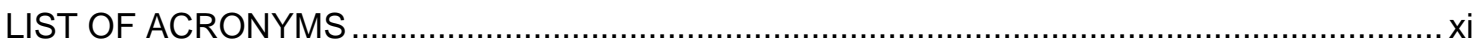

$\begin{array}{lll}\text { Chapter Page } & \text { Pand } \\ & & \end{array}$

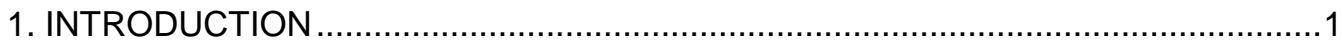

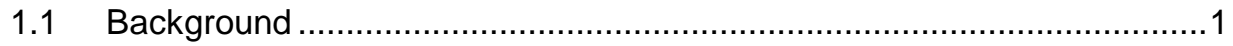

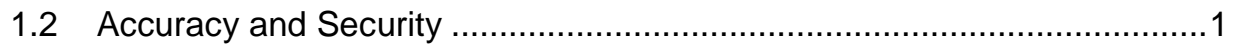

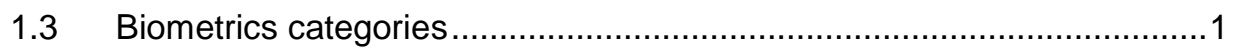

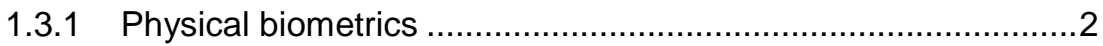

1.3.2 Behavioral biometrics ...................................................

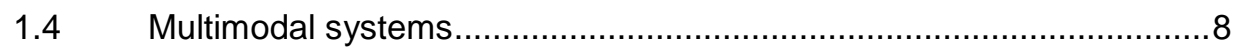

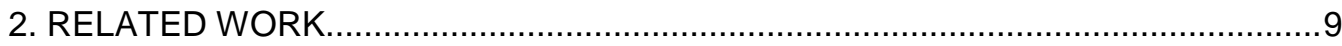

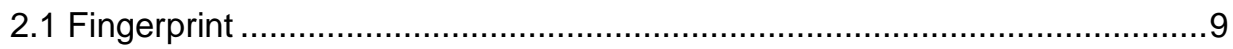

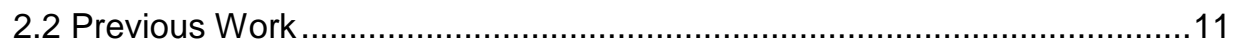

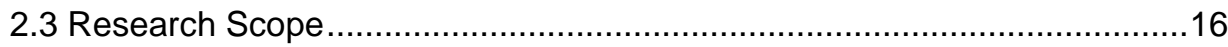

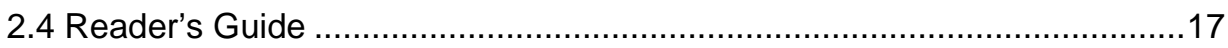

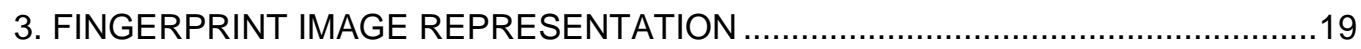

3.1 Fingerprint Representation............................................................19

3.1.1 Image-based representation .........................................21 
3.1.2 Global Ridge Pattern ..................................................22

3.1.3 Local Ridge Detail ....................................................22

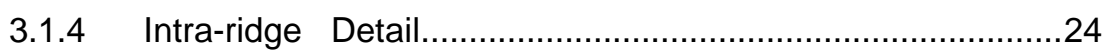

3.2 Minutiae-Based Fingerprint Recognition .......................................25

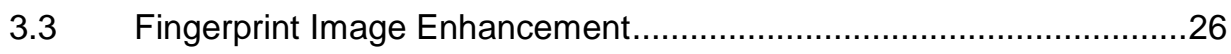

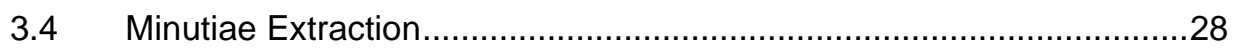

3.4.1 Binarization-based Minutiae Extraction..............................29

4. REVIEW OF THE AUTOMATIC FINGERPRINT IDENTIFICATION SYSTEM .........33

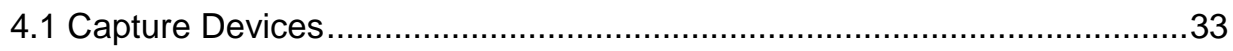

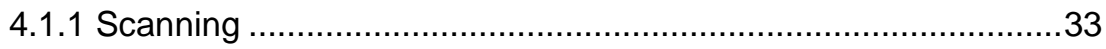

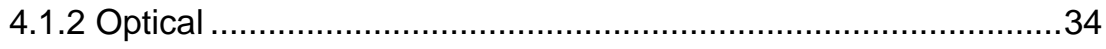

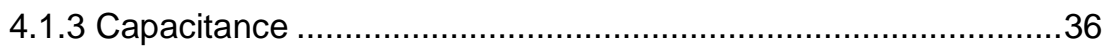

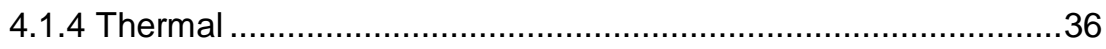

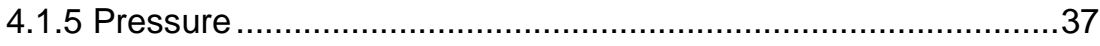

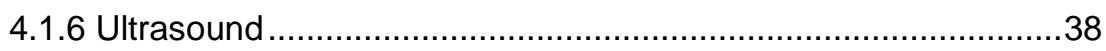

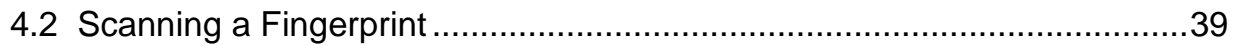

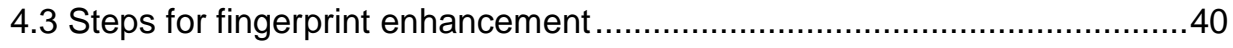

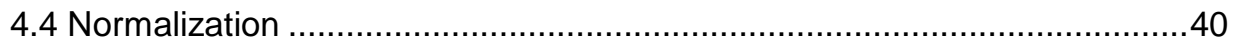

4.5 LRO (Local Ridge Orientation) …................................................... 42

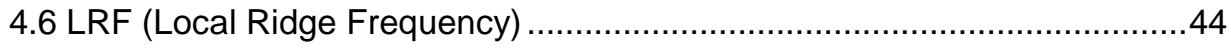

4.7 Algorithm for fingerprint enhancement ............................................. 44

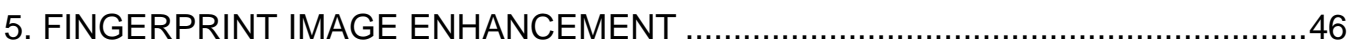

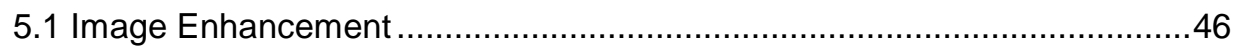

5.1.1 Orientation Estimation and Verification .................................48

5.1.2 Predicting ridge orientations using minutiae triplets ................50 
5.2 Orientation method used in this thesis .............................................52

5.2.1 LRO (Local Ridge Orientation) ............................................53

5.2.2 Algorithm for estimating LRO at a point ..................................53

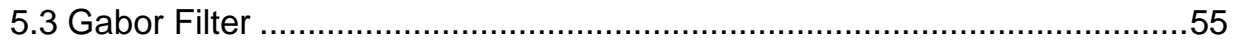

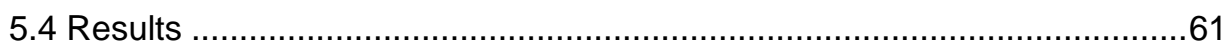

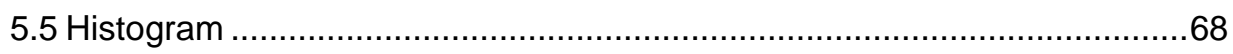

5.6 Histogram equalization................................................................ 68

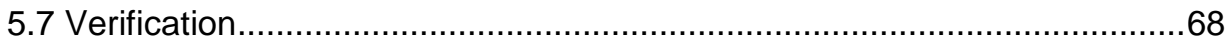

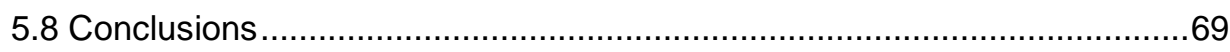

APPENDIX

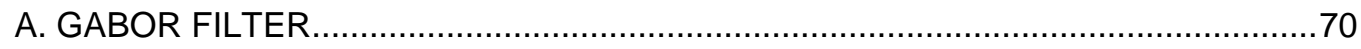

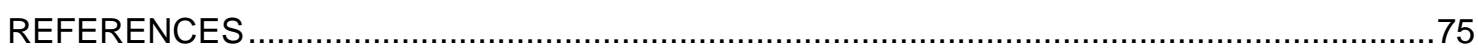

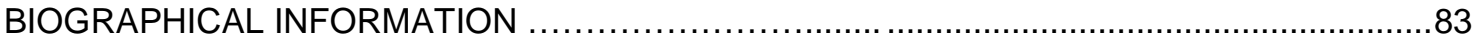




\section{LIST OF ILLUSTRATIONS}

Figure

Page

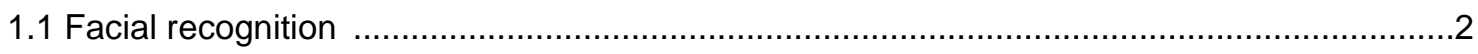

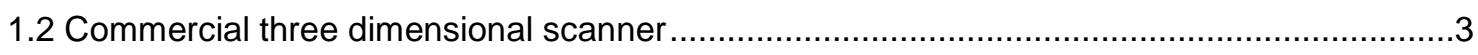

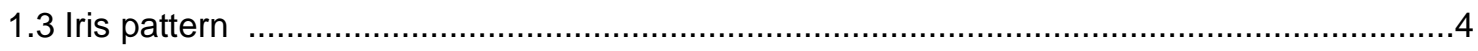

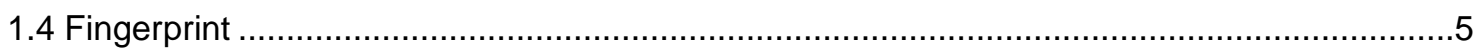

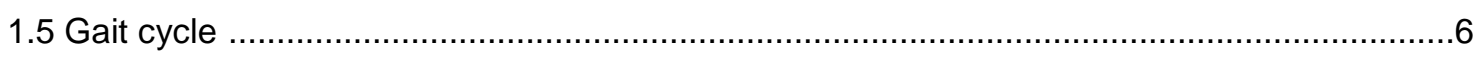

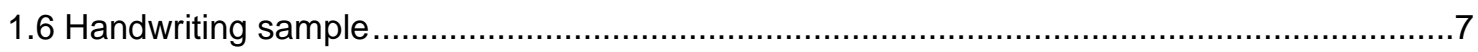

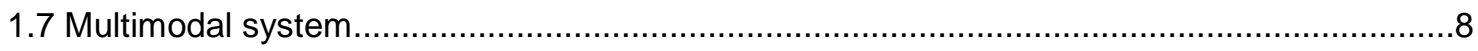

2.1 Feature at various level in fingerprint (a) Grayscale image (b) level 1 feature $\begin{array}{lll}\text { (orientation field) } & \text { (c) level } 2 \text { feature (ridge skeleton) } & \text { (d) level } 3\end{array}$ feature (ridge contour, pore and dot)

2.2 Fingerprint representation schemes (a) Grayscale image [47] (b) phase image [48] (c) skeleton image

2.3 Deducing the orientation field from minutiae distribution. (a) A single minutiae triplet. (b) Forming triplets across the minutiae distribution. (c) Estimated orientation field using minutiae triplet information

2.4 (a) Whorl (b) Left loop (c) Right loop.....

2.5 Continuous phase for a whorl pattern (a) continuous phase given by $x 2+y 2$

(b) Continuous phase modulo $2 \Pi$ (c) Gray scale image given by

$\cos (x 2+y 2)$ (d) Gradient of the continuous phase

3.1 Sample fingerprints with their associated shapes

3.2 Sample fingerprints, with core points marked with a square, and delta points marked with a triangle

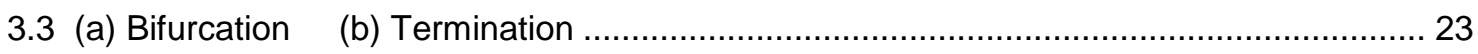

3.4 Some of the common minutiae types 
3.7 (a) The window used for analyzing the surrounding pixel intensity (b) the window oriented along the local ridge direction

4.1 Fingerprint scanner

4.2 General layout of an optical fingerprint scanner, reproduced from [Atmel Corporation 2001]

4.3 Depiction of a capacitance scanner

4.4 Operation of ultrasound scanner, sound waves return a partial echo at each change in material, from Ultra-Scan

4.5 Scanned image

4.6 A flowchart of the proposed fingerprint enhancement algorithm

4.7 Normalized image

4.8 Orientation field image

4.9 Algorithm for fingerprint enhancement

5.3 Six ridge patterns and their orientations calculated by Kirsch(1st row), Robinson(2nd row), Sobel(3rd row), and Prewitt operators(4th row)

5.4 (a) A fingerprint ridge flows with ideal corresponding histogram. (b) Six directions for histograms examination

5.5 (a) Minutiae distribution of a fingerprint. (b) Examples of a good quality triplet (blue) with $\operatorname{Lavg}=112: 66, \theta$ diff $=5, Q=237: 63$ and a bad quality triplet (red) with Lavg $=217$,_diff $=26, Q=67: 55$. (c) Estimated orientation map.....

5.6 Projections of a window of fingerprint image data. The projections which exhibit the greatest variation correspond to the orientation of the ridges within the window (here). Eight projection are shown here ....

5.7 One dimensional Gabor filter

5.8 Joint localization of a signal in time and frequency domain 
5.10 Image 1(a) Original image (b) Gabor filtered image (c) Histogram of the original image (d) Histogram of the Gabor filtered image (e) Histogram equalized of the Gabor filtered image

5.11 Image 2(a) Original image (b) Gabor filtered image (c) Histogram of the original image (d) Histogram of the Gabor filtered image (e) Histogram equalized of the Gabor filtered image

5.12 Image 3(a) Original image (b) Gabor filtered image (c) Histogram of the original image (d) Histogram of the Gabor filtered image (e) Histogram equalized of the Gabor filtered image

5.13 Image 4(a) Original image (b) Gabor filtered image (c) Histogram of the original image (d) Histogram of the Gabor filtered image (e) Histogram equalized of the Gabor filtered image

5.14 Image 5(a) Original image (b) Gabor filtered image (c) Histogram of the original image (d) Histogram of the Gabor filtered image (e) Histogram equalized of the Gabor filtered image

5.15 Image 6(a) Original image (b) Gabor filtered image (c) Histogram of the original image (d) Histogram of the Gabor filtered image (e) Histogram equalized of the Gabor filtered image

5.16 Image 7(a) Original image (b) Gabor filtered image (c) Histogram of the original image (d) Histogram of the Gabor filtered image (e) Histogram equalized of the Gabor filtered image 


\section{LIST OF ACRONYMS}

AFIS - Automatic Fingerprint Identification System

ANSI - American National Standards Institute

DB - Data Base

DMF - Directional Median Filter

DPI - Dots Per Inch

ESID - Electro-Static Discharge

FBI - Federal Bureau of Investigation

FFT - Fast Fourier Transform

FM - Frequency Modulation

FTIR - Frustrated Total Internal Reflection

FVC - Fingerprint Verification Competition

JPEG - Joint Photographic Experts Group

LRF - Local Ridge Frequency

LRO - Local Ridge Orientation

NIST - National Institute of Standards and Technology

PAMI - Pattern Analysis and Machine Intelligence

SEIR - Surface Enhanced Internal Reflection

SFINGE - Synthetic Fingerprint Generator

TAR - True Accept Rate

WSQ - Wavelet Scalar Quantization 


\section{CHAPTER 1}

\section{INTRODUCTION}

\subsection{Background}

Biometrics is the science of verifying the identity of an individual through physiological measurements or behavioral traits. Since biometric identifiers are associated permanently with the user they are more reliable than token or knowledge based authentication methods.

Biometrics offers several advantages over traditional security measures. Some of them are presented below

\subsection{Accuracy and Security}

Biometrics based security systems are far most secure and accurate than traditional password or token based security systems. For example a password based security system has always the threat of being stolen and accessed by the unauthorized user. Furthermore the traditional security systems are always prone to accuracy as compared to biometrics which is more accurate.

One individual, Multiple IDs: Traditional security systems face the problem that they don't give solution to the problem of individuals having multiple IDs, e.g. a person having multiple passports to enter a foreign country. Thanks to biometrics!!! They give us a system in which an individual cannot possess multiple IDs and cannot change his ID throughout his life time. Each individual is identified through a unique biometric identity throughout the world.

\subsection{Biometrics categories}

Biometrics can be categorized in various categories as follow. 


\subsubsection{Physical biometrics}

This biometrics involves measurement of physical characteristics of individuals. The most prominent of these include

- Face

- Hand geometry

- Iris scans

- Fingerprints

\subsubsection{Face}

There has been significant achievement in face recognition system in past few years. Due to these advancements this problem appears to be eventually technologically feasible and economically realistic. In addition, current research involves developing more robust approaches that accounts for changes in lighting, expression, and aging, where potential variations for a given person are illustrated in Fig 1-1. Also, other problem areas being investigated include dealing with glasses, facial hair, and makeup.

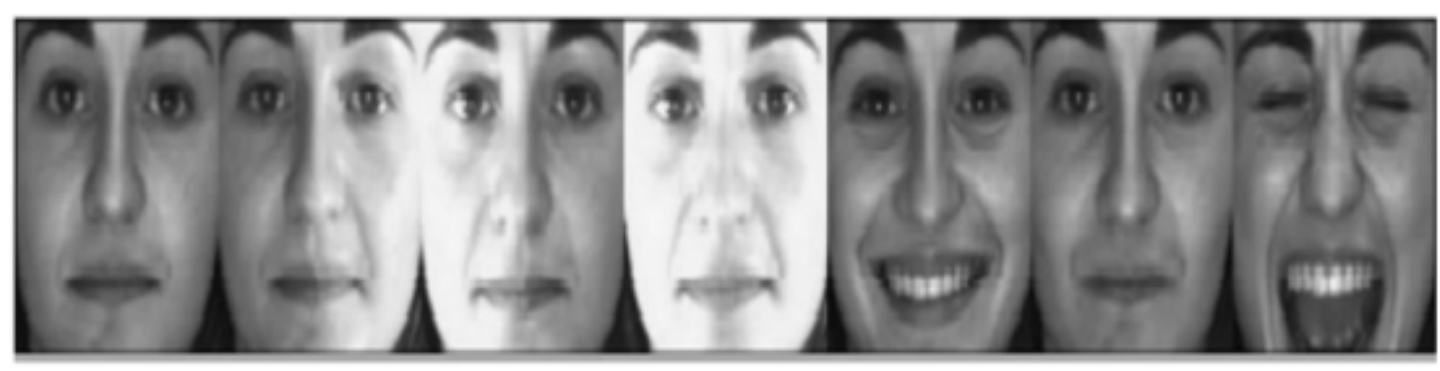

Fig 1-1 Facial recognition [66]

\subsubsection{Hand geometry}

Hand geometry is one of the most basic biometrics in use today. A two- dimensional system can be implemented with a simple document scanner or digital camera, as these 
systems only measure the distances between various points on the hand. Meanwhile, a three dimensional system provides more information and greater reliability. These systems, however, require a more expensive collection device than the inexpensive scanners that can be used in a two-dimensional system. An example of a commercial three-dimensional scanner is shown in Fig 1-2.

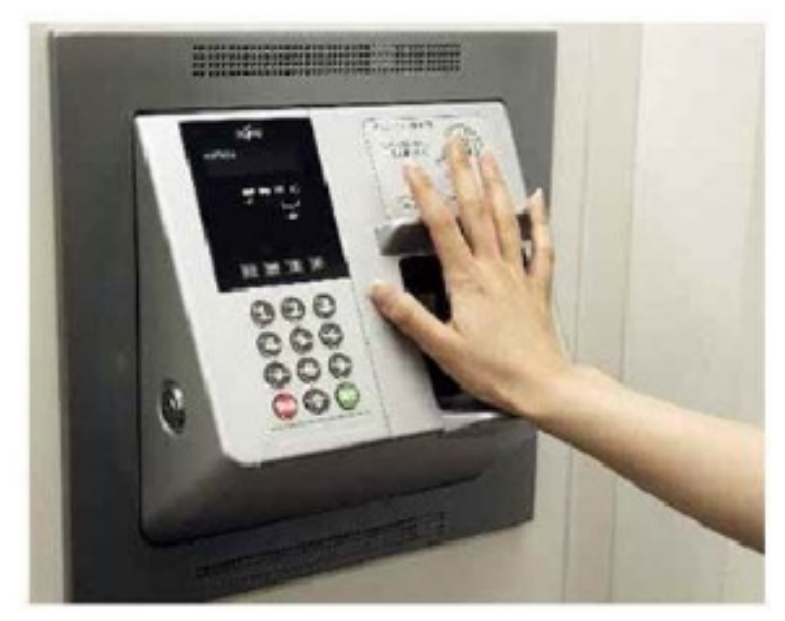

Fig 1-2 Commercial three dimensional scanner [66]

The primary advantage of hand geometry systems is that they are simple and inexpensive to use. Also, poor weather and individual anomalies such as dry skin or cuts along the hand do not appear to negatively affect the system. The geometry of the hand, however, is not a very distinctive quality. In addition, wearing jewelry or other items on the fingers may adversely affect the system's performance.

\subsubsection{Iris}

Iris recognition Fig 1-3 has taken on greater interest in recent years. As this technology advances, purchasing these systems has become more affordable. These systems are 
attractive because the pattern variability of the iris among different persons is extremely large. Thus, these systems can be used on a larger scale with a small possibility of incorrectly matching an imposter. Also, the iris is well protected from the environment and remains stable over time. In terms of localizing the iris from a face, its distinct shape allows for precise and reliable isolation. Fig 1-3 shows the unique iris pattern data extracted from a sample input.

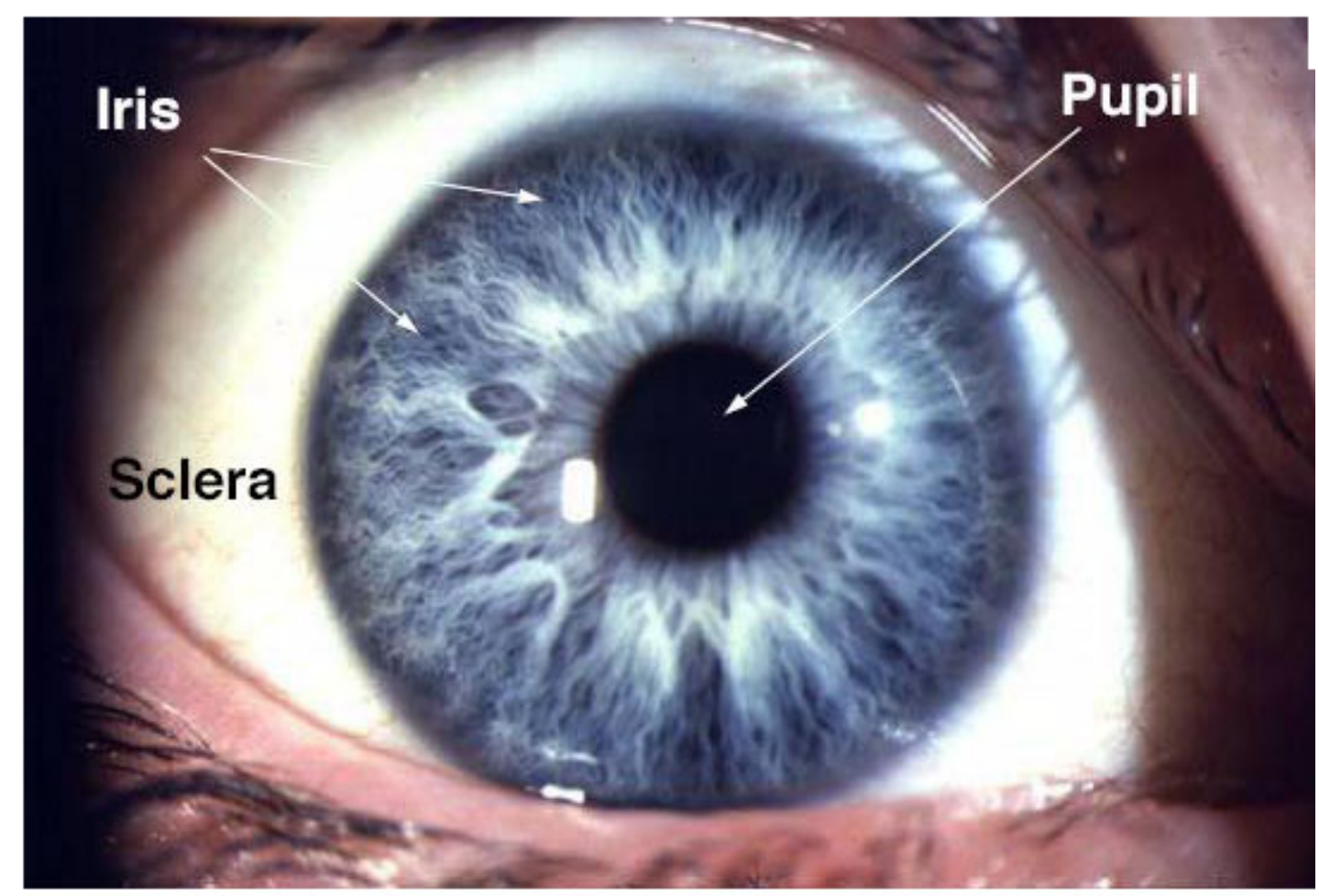

Fig 1-3 Iris pattern [84]

\subsubsection{Fingerprint}

Fingerprint features are very important in fingerprint recognition process. Fingerprint features are generally categorized into three levels (Fig 1-4)

1. Level 1 features mainly refer to ridge orientation field and features derived from it, i.e., singular points and pattern type.

2. Level 2 features refer to ridge skeleton and features derived from it, i.e., ridge 
bifurcations and endings.

3. Level 3 features include ridge contours, position, and shape of sweat pores and incipient ridges.

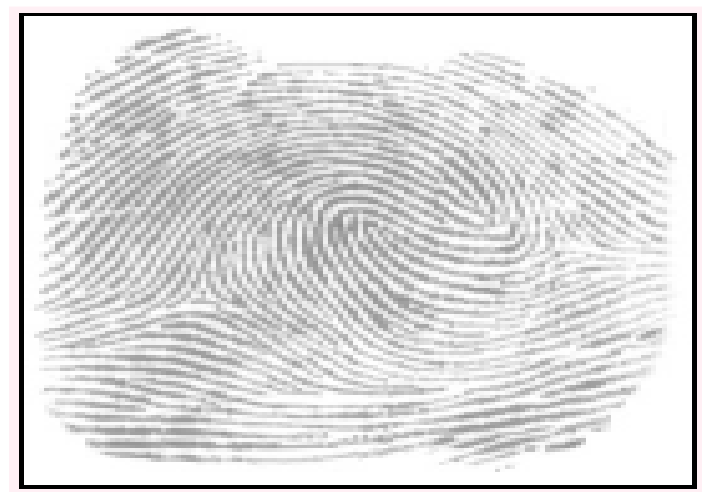

Fig 1-4 Fingerprint

\subsubsection{Behavioral biometrics}

This category of biometrics is temporal in nature. They are evolved during the life time of an individual. It involves measuring the way in which an individual performs certain tasks. Behavioral biometrics include

- Gait

- Handwriting

- Speech

- Signature

Now let discuss some the behavioral biometrics in a little detail

\subsubsection{Gait}

Gait-based recognition involves identifying a person's walking style. Although these systems are currently very limited, there is a significant amount of research being conducted in this area. Furthermore, studies have shown that gait changes over time and is also affected by 
clothes, footwear, walking surfaces, and other conditions. Figure below outlines the various stages of a gait cycle (Fig 1-5).
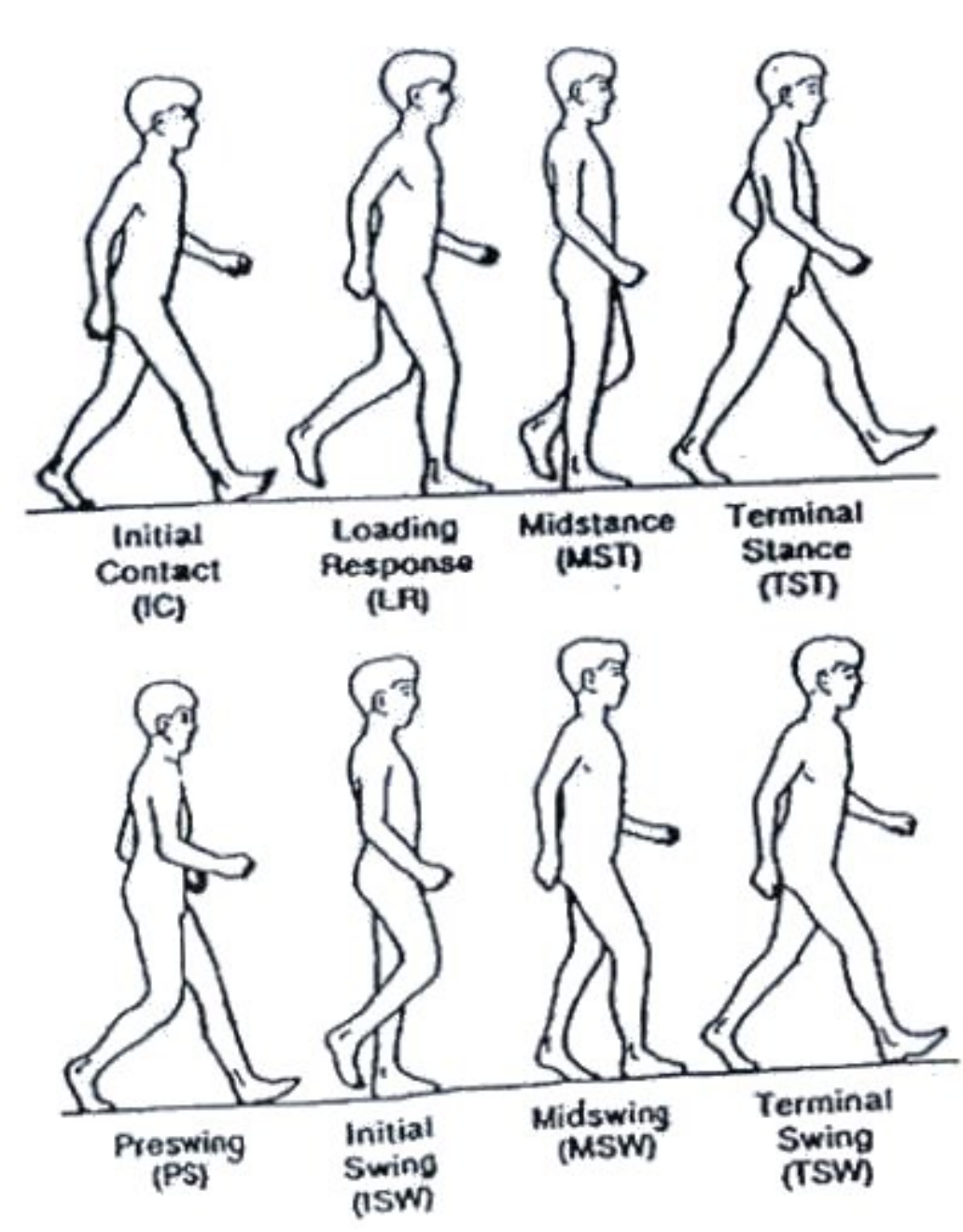

Fig 1-5 Gait cycle [85]

\subsubsection{Handwriting}

Signature verification, for example, has had a long, rich history. The use of signatures has some well known advantages: they are a natural and familiar way of confirming identity, have already achieved acceptance for legal purposes, and their capture is less invasive than most other biometric schemes [70]. Still, each individual has only one true signature Fig 1-6 -a 
severe limitation when it comes to certain security applications. As a result, researchers have recently begun to examine using arbitrary handwritten phrases, recasting the problem as one of computing cryptographic keys or biometric hashes (e.g., [71]).

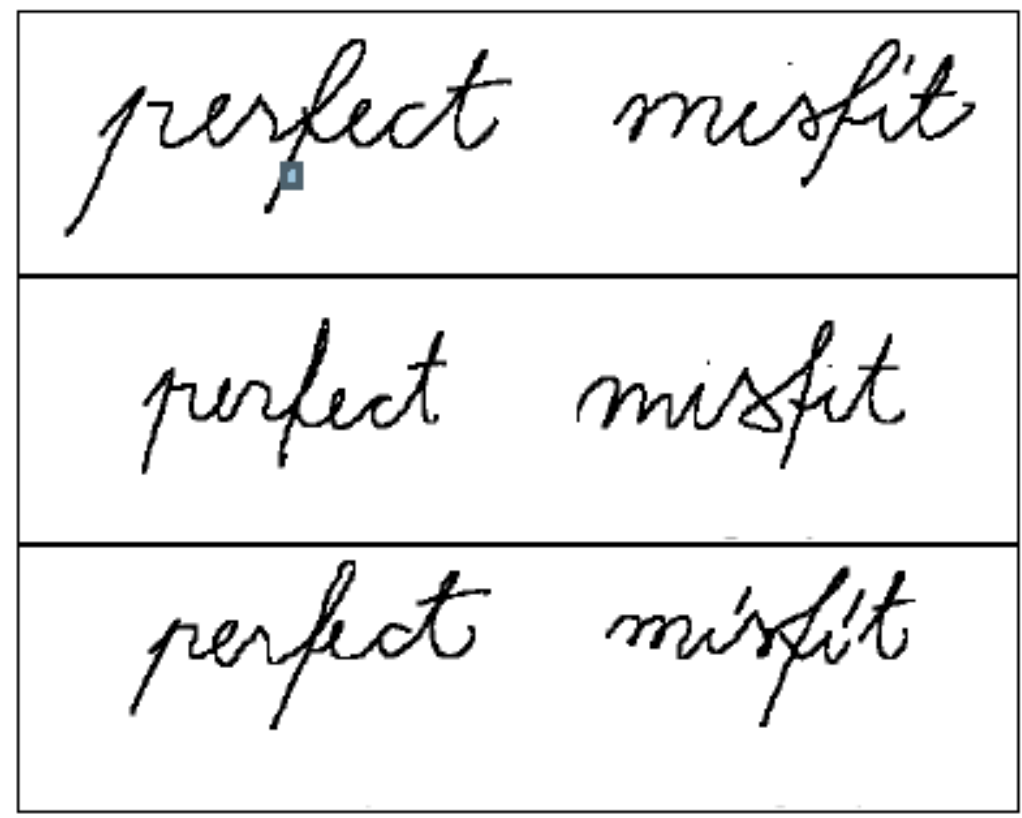

Fig 1-6 Handwriting sample

\subsubsection{Speech}

Voice, as one of the modalities, is especially important in applications such as telephony dialog systems where it is the natural communication means and, besides the dialing keyboard, the only one available. Speaker recognition technology analyzing and modeling the speaker's voice prints has been a major research effort for the past decades and is reaching maturity.

\subsubsection{Signature}

Signature characteristics are absolutely unique to an individual and virtually impossible to duplicate. Therefore, signature still remains one of the most powerful human identifiers today. 
In dynamic signature verification, multiple biometric characteristics of a signature in question are scrutinized and compared against a reference signature kept on file to make a conclusion that measures the confidence of the signature's genuineness. If several genuine reference signatures are available, the measure of the stability of the particular feature is developed and used to estimate the probability of deviations observed in the questionable signature.

\subsection{Multimodal systems}

Multimodal systems (Fig 1-7) employ more than one biometric recognition technique to arrive at a final decision. These systems may be necessary to ensure accurate performance. Combining several biometrics in one system allows for improved performance as each individual biometric has its own strengths and weaknesses. Using more than one biometric also provides more diversity in cases where it is not possible to obtain a particular characteristic for a person at a given time. Although acquiring more measurements increases the cost and computational requirements, the extra data allows for much greater performance.

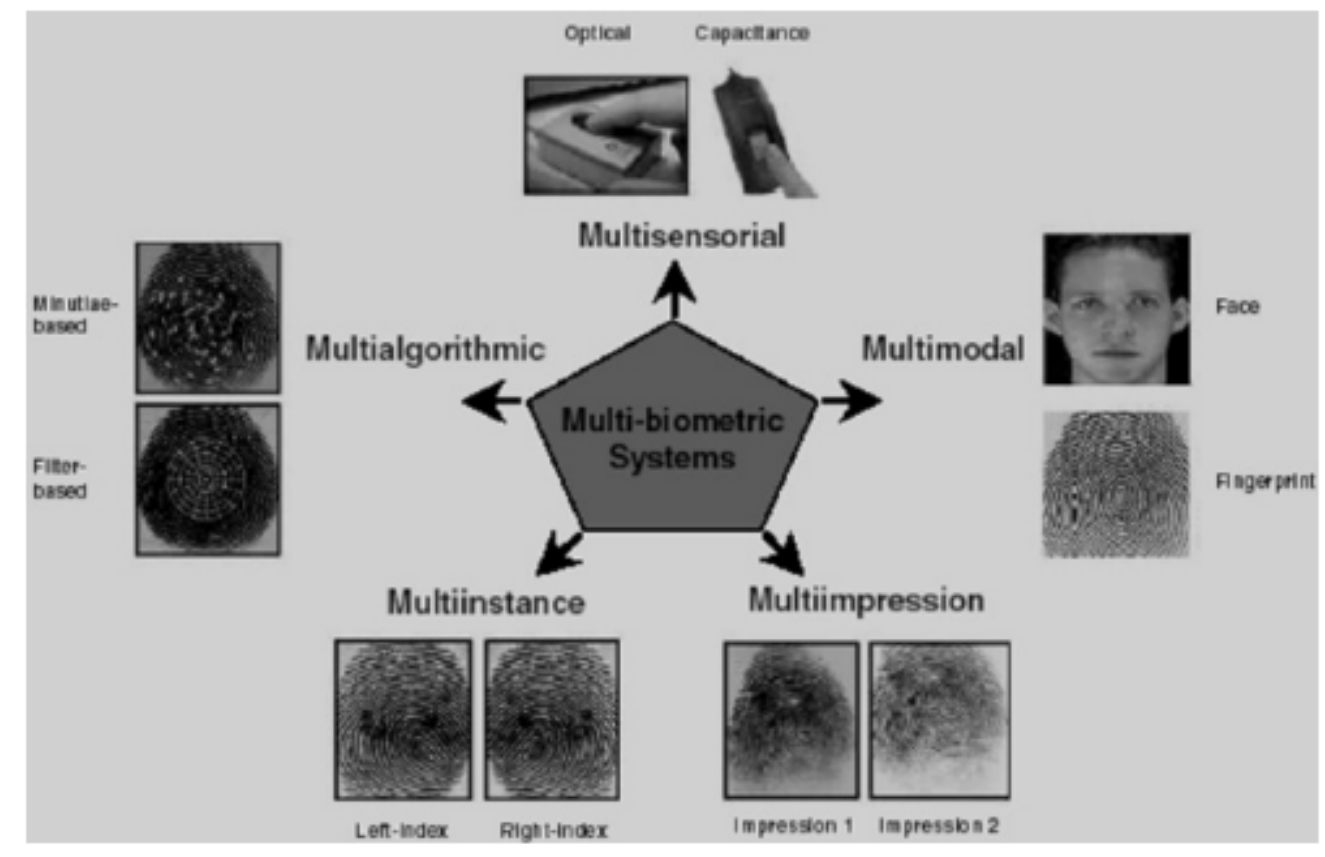

Fig 1-7 Multimodal system 


\section{CHAPTER 2}

\section{RELATED WORK}

Security, in general terms, is concerned with the protection of some kind of asset. The level of security protecting these assets is relative to that provided by other (similar) systems, and to the ease with which an attacker can gain access to the asset. The introduction of biometric systems as an alternative to traditional security systems is seen as attractive by many, because of the potential for greater precision. The ability to use a characteristic of the user as a means of identification or authentication is seen as a benefit in terms of both security and usability. However the use of biometric systems introduces new risks both to the system and the user. Biometric characteristics as used in biometric systems are typically consistent elements of what a person is, and hence cannot be easily altered or replaced. Therefore the theft of such a characteristic, especially if it is used as a key to a secure environment, is potentially devastating. Various previous works related to security and systems are mentioned in this chapter.

\subsection{Fingerprint}

Extensive research has been done on fingerprints. Two of the fundamentally important conclusions that have risen from research are:

(1) a person's fingerprint will not naturally change structure about one year after birth and

(2) the fingerprints of individuals are unique. Even the fingerprints in twins are not the same. In practice two humans with the same fingerprint have never been found.

Fingerprint features are generally categorized into three levels (Fig 2-1): 
1. Level 1 features mainly refer to ridge orientation field and features derived from it, i.e., singular points and pattern type.

2. Level 2 features refer to ridge skeleton and features derived from it, i.e., ridge bifurcations and endings.

3. Level 3 features include ridge contours, position, and shape of sweat pores and incipient ridges.

Most fingerprint matching systems are based on four types of fingerprint representation schemes: grayscale image, phase image, skeleton image, and minutiae

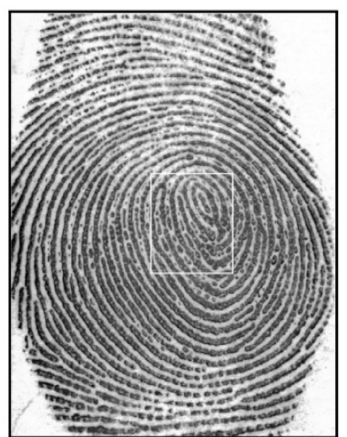

(a)

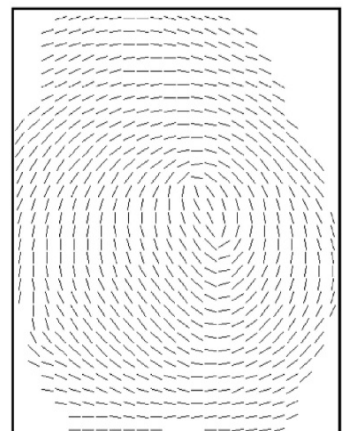

(b)

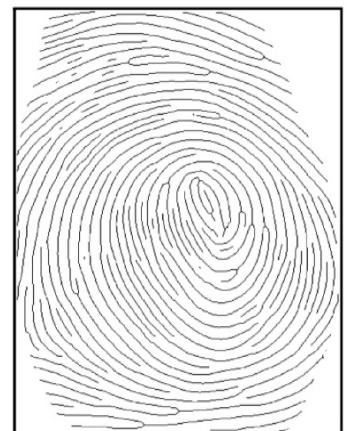

(c)

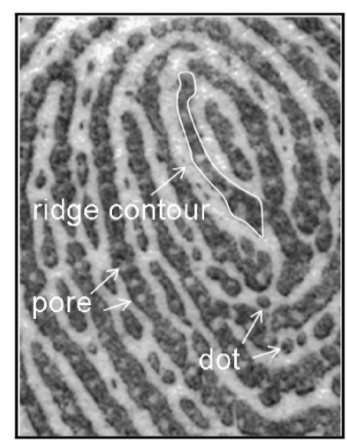

(d)

Fig 2-1 Feature at various level in fingerprint (a) Grayscale image (b) level 1 feature (orientation field) (c) level 2 feature (ridge skeleton) pore and dot) [53]

(d) level 3 feature (ridge contour,

The gray scale image (fig.2.2(a)) has the highest amount of information and features at all levels are represented. Compared to grayscale image, phase image (Fig 2-2(b)) and skeleton image (Fig 2-2(c)) lose all Level 3 features and compared with phase image and skeleton image, the minutiae template further loses some Level 2 information, such as ridge path between minutiae. 


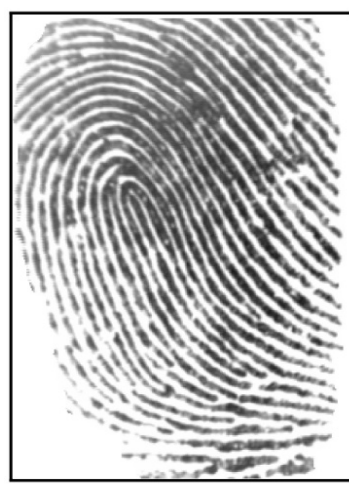

(a)

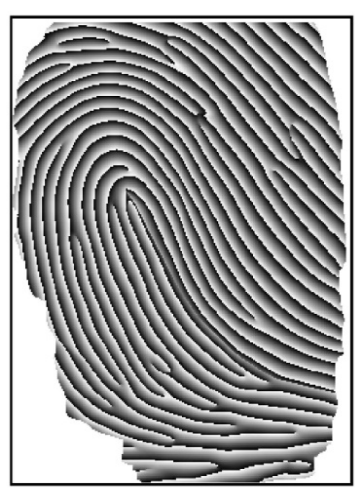

(b)

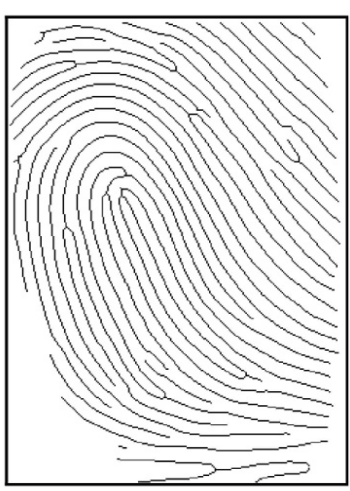

(c)

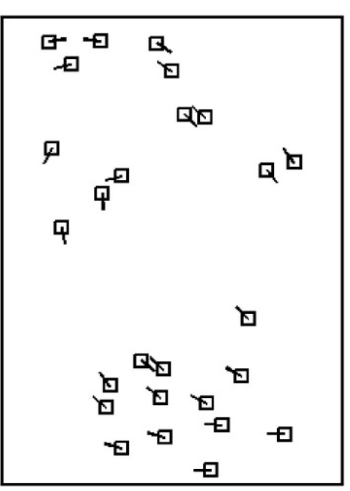

(d)

Fig 2-2 Fingerprint representation schemes (a) Grayscale image [47] (b) phase image [48] (c) skeleton image[49],[50] (d) minutiae [51],[52]

\subsection{Previous work}

The methods of Hill [54] and Ross et al [55] first reconstructs a skeleton image from minutiae, which is then converted into the grayscale image. In [54], the orientation field is generated based on singular points according to the model in [56]. A line drawing algorithm is used to generate a sequence of splines passing through the minutiae.

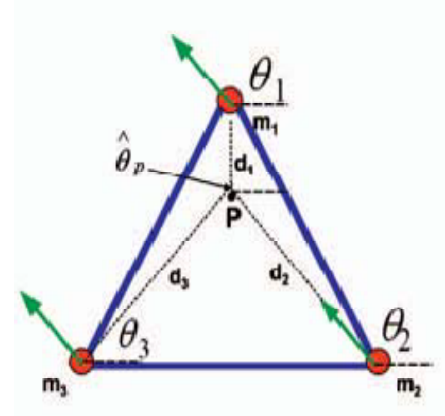

(a)

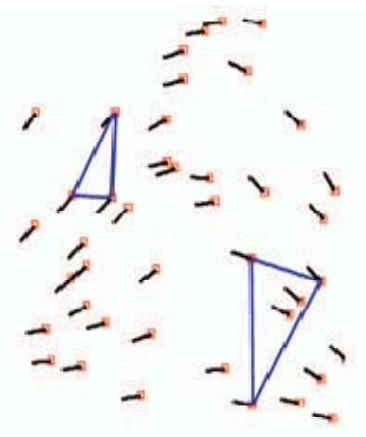

(b)

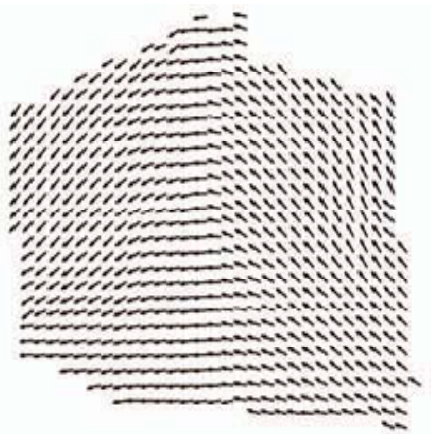

(c)

Fig 2-3 Deducing the orientation field from minutiae distribution. (a) A single minutiae triplet. (b) Forming triplets across the minutiae distribution. (c) Estimated orientation field using minutiae triplet information [55]

In [55], the orientation field is estimated using selected minutiae triplets in the template. Streamlines are then traced starting from minutiae and border points. Linear integral convolution 
is used to impart texture-like appearance to the ridges. Finally, the image is smoothed to obtain wider ridges.

Consider a pixel $\mathrm{P}(\mathrm{x}, \mathrm{y})$ located inside the triangular region defined by a triplet.

Let $d(i)=\operatorname{dist}\left\{m_{i}, P\right\}, i=1,2,3$ be the Euclidean distances of this pixel from the entire ith vertex. The orientation of the pixel $\mathrm{P}, \widehat{\theta \hat{\theta}_{p}}$, is then estimated as [55]

$$
\theta \widetilde{\theta_{p}}=\frac{d_{3} d_{2}}{d_{s} d_{2}+d_{s} d_{1}+d_{1} d_{2}} \theta \theta_{1}+\frac{d_{1} d_{3}}{d_{s} d_{2}+d_{s} d_{1}+d_{1} d_{2}} \theta \theta_{2}+\frac{d_{1} d_{2}}{d_{s} d_{2}+d_{s} d_{1}+d_{1} d_{2}} \theta \theta_{3}
$$

The angle $\theta_{1}\left(\theta_{3}\right)$ corresponds to the orientation of the vertex that is nearest to (farthest from) the pixel $P(x, y)$. Thus, the orientation at $P(x, y)$ is estimated as a weighted sum of all the three orientations with a higher weight assigned to the orientation of the closest vertex. The result of the generated orientation map is shown in Fig 2-3.

Averaging orientation map: To obtain a smooth transition in orientations, the estimated orientation map is convolved with a $3 \times 3$ local averaging filter.

This reconstruction algorithm [55] can only generate a partial fingerprint. In addition, streamlines that terminate due to distance constraint between adjacent streamlines will generate spurious minutiae. The validity of this reconstruction algorithm was tested by matching 2,000 reconstructed fingerprints against the 2,000 original fingerprints in NIST SD4. A rank-1 identification rate of 23 percent was reported.

Cappelli et al [57] proposed a technique to directly reconstruct the grayscale image from minutiae. The orientation field is estimated by fitting a modified model initially proposed in [58] to the minutiae directions. Gabor filtering [65] is iteratively performed starting from minutiae on an image initialized by the local minutiae pattern. A rendering step is performed to make the reconstructed fingerprint image appear more realistic. The efficacy of this reconstruction 
algorithm was assessed by attacking nine fingerprint matching algorithms. An average True Accept Rate (TAR) of 81.49 percent at 0 percent False Accept Rate (FAR) was obtained in matching 120 reconstructed finger-prints against the 120 original fingerprints in FVC2002 DB1. However, this algorithm also generates many spurious minutiae in the reconstructed fingerprints.

Fingerprint reconstruction from minutiae (hereinafter simply referred to as fingerprint reconstruction) is very similar to fingerprint synthesis except that the goals and the inputs of the two techniques are different. The goal of fingerprint reconstruction is to obtain an artificial fingerprint that resembles the original fingerprint as much as possible, while the goal of fingerprint synthesis is to generate any artificial fingerprint that is as realistic as possible. For fingerprint reconstruction, the minutiae from a given fingerprint must be provided, while for fingerprint synthesis, no input is needed (except for a statistical model of fingerprint learned from many real fingerprint images).

The well-known SFINGE [69] fingerprint synthesis method of Cappelli et al performs Gabor filtering on a seed image according to the orientation and frequency images; minutiae automatically emerge during the filtering procedure. Some intraclass variations, such as spatial transformation, touching area, nonlinear distortion, ridge dilation/shrinking, and noise, are simulated to generate realistic impressions of the master fingerprint. One main limitation of SFINGE is that minutiae cannot be controlled. As a result, SFINGE may generate problematic fingerprints that contain too few minutiae or very long ridges. It is well known that the distribution of minutiae in fingerprints is not random and fingerprints of different pattern types have different minutiae distributions [55]. The minutiae distribution of fingerprints generated by SFINGE may not conform to such distributions since these minutiae are automatically generated during the image filtering process. Similar fingerprint synthesis methods have also been proposed in [60]. The reaction-diffusion technique described in [62] can also be used for synthesizing fingerprints. 
Bicz [61] described a fingerprint synthesis technique based on the 2D FM model. The phase of the FM model consists of the continuous component and the spiral component, which corresponds to minutiae. A fingerprint is synthesized by first generating each component separately and then combining them. Separation of the continuous phase and the spiral phase makes minutiae controllable. However, the most important step, generating the continuous phase component, was not described in [61]. According to the demo software provided by the author, only a partial fingerprint (around the core) can be generated and the orientation field of each of the four fingerprint pattern types (whorl, left loop, right loop, and arch (Fig 2-4) is fixed.

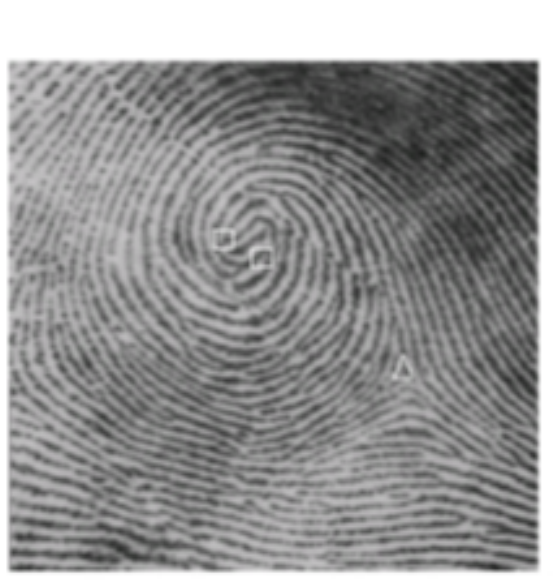

(a)

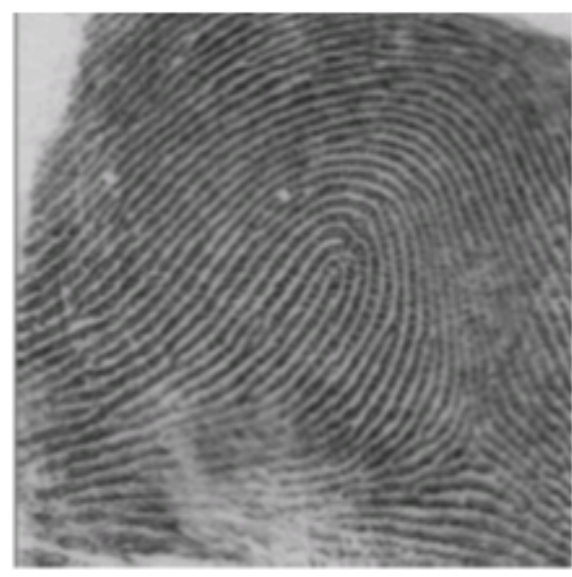

(b)

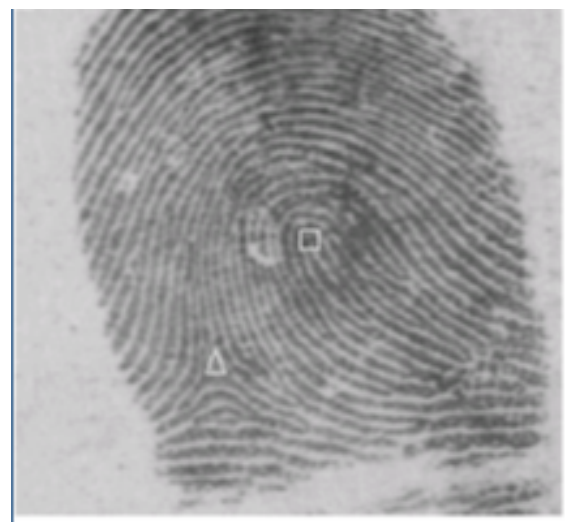

(c)

Fig 2-4 (a) Whorl (b) Left loop (c) Right loop 
Feng, and Jain [53] proposed a novel approach to fingerprint reconstruction from minutiae template which first reconstructs a phase image from the minutiae template and then converts the phase image into the grayscale image. The advantages of this approach over existing approaches to fingerprint reconstruction [54], [55], [57] are: 1) A complete fingerprint can be reconstructed and 2) the reconstructed fingerprint contains very few spurious minutiae. The proposed reconstruction algorithm has been quantitatively assessed by matching reconstructed fingerprints against the corresponding original fingerprints (termed as type-I attack) and against different impressions of the original fingerprints (termed as type-Il attack) using a commercial fingerprint SDK, Neurotechnology VeriFinger 4.2. Type-I attack was found to have a high chance of deceiving the fingerprint recognition system in both the verification and identification experiments. Type-II attack also has a significantly higher acceptance rate than that of impostor match. A TAR of 94.13 percent at a FAR of 0 percent has been observed in the verification experiment conducted on FVC2002 DB1, and 99.70 percent rank-1 identification rate has been observed in the identification experiment conducted on the NIST SD4 database.

The continuous phase does not contain any rotational component and the integral of its gradient around any simple closed path is zero. For example, the continuous phase given by

$$
\psi_{c}(x, y)=\sqrt{x^{2}+y^{2}}
$$

corresponds to a grayscale image $\cos \left(\psi_{\mathrm{c}}(\mathrm{x}, \mathrm{y})\right)$ that looks like a whorl pattern (see Fig 2-5). Its gradient (instantaneous frequency) is $\cos \theta$ and $\sin \theta$, where $\theta$ is the angle in the polar coordinate system. 


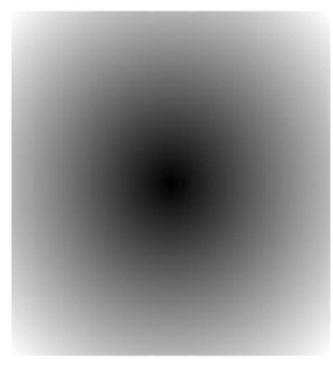

(a)

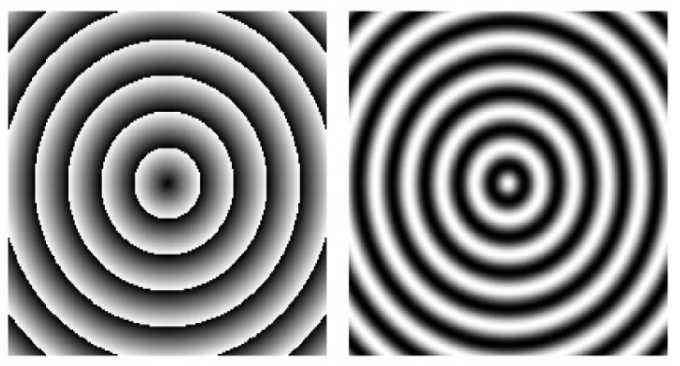

(b) (c)

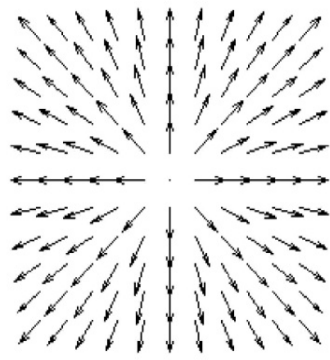

(d)

Fig 2-5 Continuous phase for a whorl pattern (a) continuous phase given by $\sqrt{x^{2}+y^{2}}$ (b) Continuous phase modulo $2 \Pi$ (c) Gray scale image given by $\cos \left(\sqrt{x^{2}+y^{2}}\right)$ (d) Gradient of the continuous phase[53]

Generally, a fingerprint based biometric system is considered as highly secure, and is equivalent to a long password. However with decreasing number of features on a small fingerprint and the non-exact matching nature, the security strength of partial fingerprint recognition reduces. Fingerprint recognition is being widely applied in the personal identification for the purpose of high degree of security. However, some fingerprint images captured in various applications are poor in quality, which corrupts the accuracy of fingerprint recognition. Consequently, fingerprint image enhancement is usually the first step in most automatic fingerprint identification systems (AFISs).

\subsection{Research Scope}

Fingerprint image enhancement is a common and critical step in fingerprint recognition systems. To enhance the images, most of the existing enhancement algorithms use filtering techniques that can be categorized as isotropic and anisotropic according to the filter kernel. Isotropic filtering can properly preserve features on the input images but can hardly improve the quality of the images. On the other hand, anisotropic filtering can effectively remove noises from the image but only when a reliable orientation is provided.

It is obvious that fingerprints are the most widely applied biometric identifiers. With the help of high performance computers, Automatic Fingerprint Identification Systems (AFIS) have 
gradually replaced human experts in fingerprint recognition as well as classification. However, fingerprint images contain noises caused by factors such as dirt, grease, moisture, and poor quality of input devices and are one of the noisiest image types, according to O'Gorman [11]. Therefore, fingerprint enhancement has become a necessary and common step after image acquisition and before feature extraction in the AFIS.

Various ridge frequency and minutiae types should be used to reconstruct images that are even more consistent with the original fingerprints. The acceptance rate reported in various papers for fingerprint enhancement can be further improved by reducing the image quality around the spurious minutiae. To reduce the risk of attacks using reconstructed fingerprints, robust fingerprint template security [63] and spoof detection techniques [64] should be developed.

In this thesis by applying a bank of Gabor filters [65] on input fingerprint images, orientation field from a set of filtered images can be estimated. Automatic fingerprint matching depends on the comparison of these local ridge characteristics and their relationships leading to personal identification.

\subsection{Reader's Guide}

The remainder of the document is structured as follows:

Chapter 2: Various previous works related to security and systems are mentioned in this chapter

Chapter 3: In this chapter various fingerprint representations are introduced and general review of image enhancement, feature extraction, and matching techniques that are used in fingerprint recognition systems are provided. 
Chapter 4: The following sections describe the components and algorithms that make up a typical fingerprint recognition system

Chapter 5: Image enhancement techniques may be grouped as either subjective enhancement or objective enhancement. This chapter gives a detailed knowledge about the technique. 


\section{CHAPTER 3}

\section{FINGERPRINT IMAGE REPRESENTATION}

In this chapter various fingerprint representations are introduced and general review of image enhancement, feature extraction, and matching techniques that are used in fingerprint recognition systems are provided. A fingerprint is the impression resulting from the friction ridges on the outer surface of the skin on a finger or thumb. While an in depth analysis of the way that fingerprints are formed is not within the scope of this thesis, it is commonly assumed within fingerprint biometric circles that no two people have the same fingerprints. A corollary to this assumption is that given a fingerprint, the information contained within is sufficient to uniquely identify a single individual. The validity of these assumptions is also outside the scope of this thesis. The ridges and interleaving valleys that constitute a fingerprint create two levels of detail that can be observed. The high level detail is the overall shape that is formed by the ridges.

\subsection{Fingerprint Representation}

There are mainly three different kinds of fingerprint representations that are used in fingerprint recognition systems and each has its own advantages and drawbacks.

When observing the patterns that the ridges of a fingerprint form together, Sir Edward Henry [54] created a classification of fingerprints into five classes. These classes are, arch, tented arch, left loop, right loop and whorl. Samples of these fingerprint shapes can be seen in Fig 3-1. 


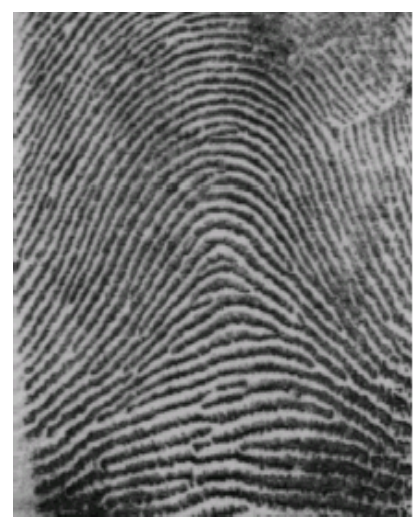

Aich

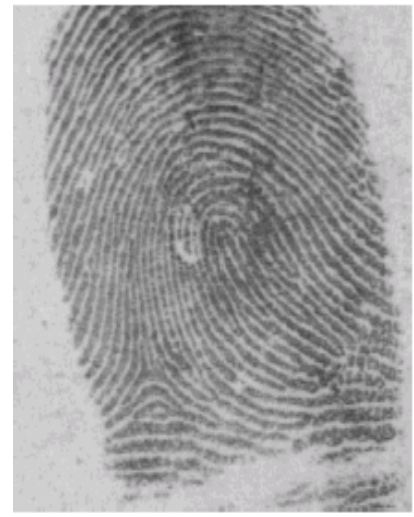

Right Loop

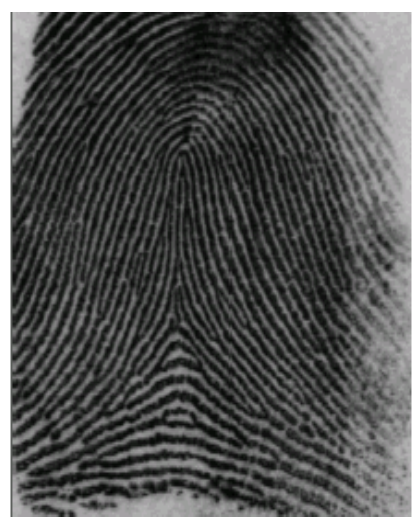

Tented Arch

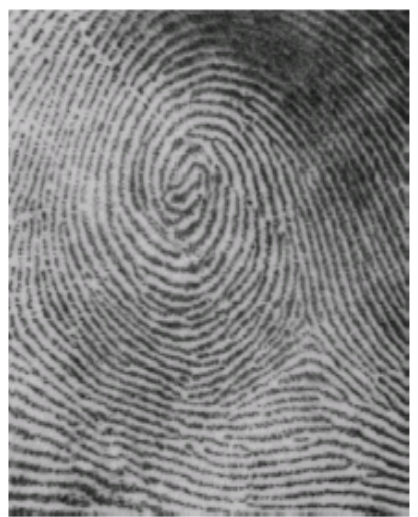

Whorl

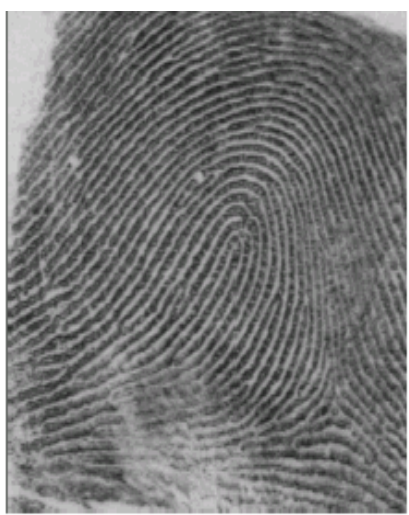

Left Loop

Fig 3-1 Sample fingerprints with their associated shapes [54]

There are two main features that define the shape of a fingerprint. These are cores and deltas (also collectively known as macro-singularities). A core is often described as a point where a single ridge line turns through 180 degrees. Similarly, a delta is described as a point where three ridge lines form a triangle. It can be seen in Fig 3-2 where the cores and deltas are marked. These core and delta points characterize the overall shape. Arches can be easily identified through the lack of any delta or core points. Also, whorls can be easily identified through the presence of two core and two delta points. Differentiating the right loop, left loop and tented arch is slightly more difficult, as all three have one core and one delta point. 


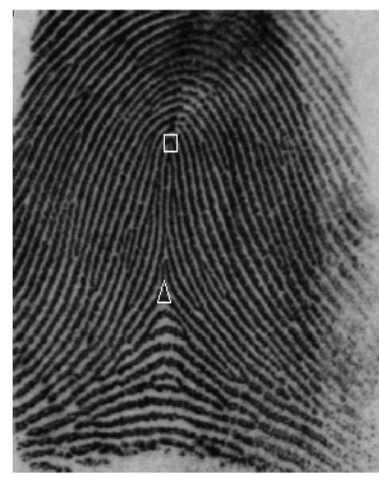

Tented Arch

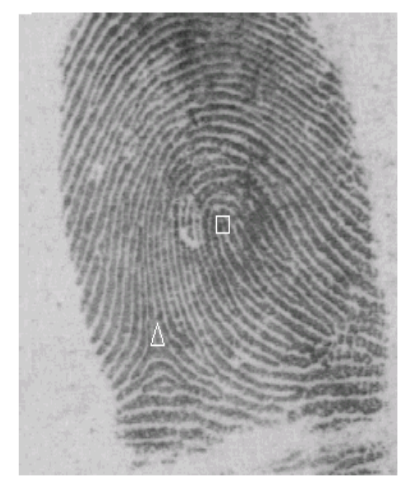

Right Loop

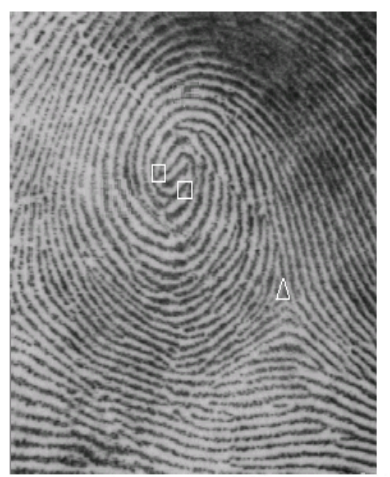

Whorl

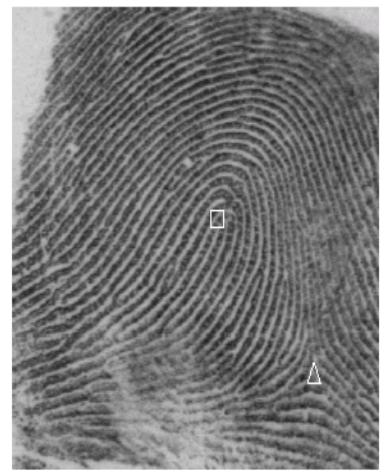

Left Loop

Fig 3-2 Sample fingerprints, with core points marked with a square, and delta points marked with a triangle [54]

\subsubsection{Image-based representation}

In image based representation, the fingerprint image itself is used as a template. There is no need for a specific feature extracting algorithm, and the raw intensity pixel values are directly used. This representation retains the most information about a fingerprint since fewer assumptions are made about the application. However, a fingerprint recognition system that uses the image-based representation requires tremendous storage space. For example, a $0.8 \mathrm{~mm} \times 1.0 \mathrm{~mm}(400 \times 500$ pixels $)$ fingerprint is obtained by a scanner at 500 dots per inch (DPI) with 8 bits gray-scale resolution. The resulting fingerprint image is $400 \times$ $500 \times 8=0.2$ Mbytes. A system with large amount of fingerprint data may have 
difficulty storing all the templates. For example, FBI has collected more than 200 million fingerprints since 1924 which require more than 250 terabytes storage space [10]. Traditional compression techniques, such as JPEG [46] tend to lose the highest frequency details, which contain discriminating information and the blocking artifacts also affect the performance of automatic fingerprint recognition systems. FBI recommends a compression method based on WSQ (Wavelet Scalar Quantization) [10], which can preserve the discriminating information without blocking artifacts while achieving a high compression ratio (around 20:1). However, it still requires about 20 Kbytes to store a compressed fingerprint image.

\subsubsection{Global Ridge Pattern}

This representation relies on the ridge structure, global landmarks and ridge pattern characteristic, such as the singular points, ridge orientation map, and the ridge frequency map. This representation is sensitive to the quality of the fingerprint images [11]. However, the discriminative abilities of this representation are limited due to absence of singular points.

\subsubsection{Local Ridge Detail}

This is the most widely used and studied fingerprint representation. Local ridge details are the discontinuities of local ridge structure referred to as minutiae. Sir Francis Galton (1822-1922) was the first person who observed the structures and permanence of minutiae. Therefore, minutiae are also called "Galton details". They are used by forensic exports to match two fingerprints.

There are about 150 different types of minutiae [11] categorized based on their configuration. Among these minutia types, "ridge ending" and "ridge bifurcation" are the most used, since all other types of minutiae can be seen as the combinations of "ridge endings" and "ridge bifurcations". 
After the fingerprint ridge thinning, marking minutia points is relatively easy. In general, for each $3 \times 3$ window, if the central pixel is 1 and has exactly 3 one-value neighbors, then the central pixel is a ridge branch as shown in figure. If the central pixel is 1 and has only 1 one-value neighbor, then the central pixel is a ridge ending as shown in Fig 3-3

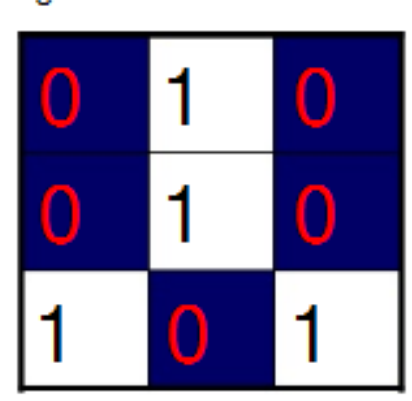

(a)

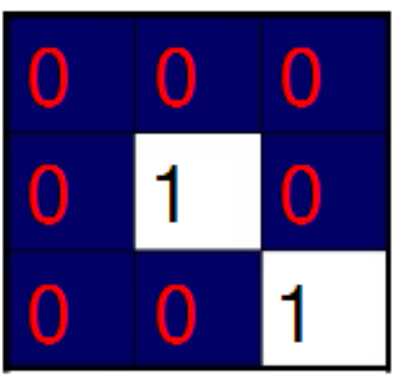

(b)

Fig 3-3 (a) Bifurcation (b) Termination

The American National Standards Institute-National Institute of Standards and Technology (ANSI-NIST) [12] proposed a minutiae-based fingerprint representation. It includes minutiae location and orientation [12]. The minutiae orientation is defined as the direction of the underlying ridge at the minutiae location. Minutiae-based fingerprint representation also has an advantage in helping privacy issues since one cannot reconstruct the original image from using only minutiae information. Minutiae is relatively stable and robust to contrast, image resolutions, and global distortion when compared to other representations. However, to extract the minutiae from a poor quality image is not an easy task.

At present, most of the automatic fingerprint recognition systems are designed to use minutiae as their fingerprint representations. 


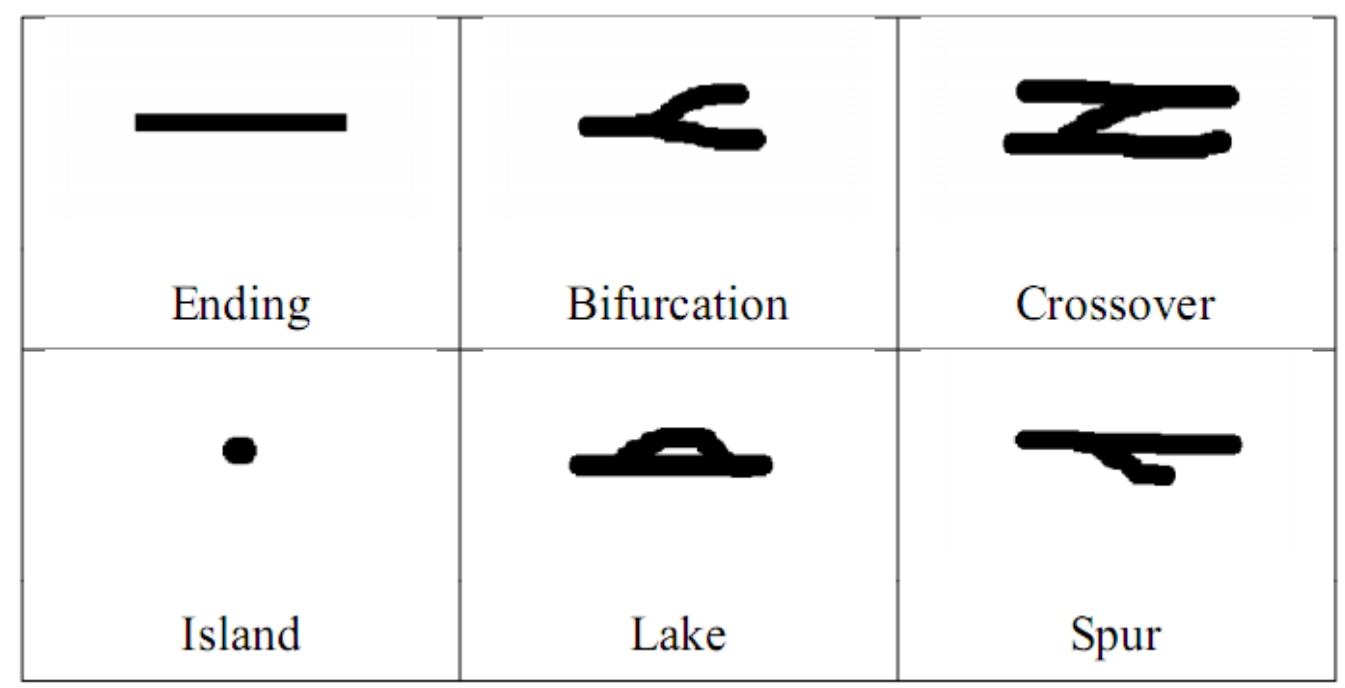

Fig 3-4 Some of the common minutiae types [59]

Some of the minutiae types are described in Fig 3-4[59]

\subsubsection{Intra-ridge Detail}

On every ridge of the finger epidermis, there are many tiny sweat pores (Fig 3-5). Pores are considered to be highly distinctive in terms of their numbers, positions, and shapes. However, extracting pores is feasible only in high-resolution fingerprint images (for example $1000 \mathrm{DPI}$ ) and with good image quality. Therefore, this kind of representation is not practical for most applications. 


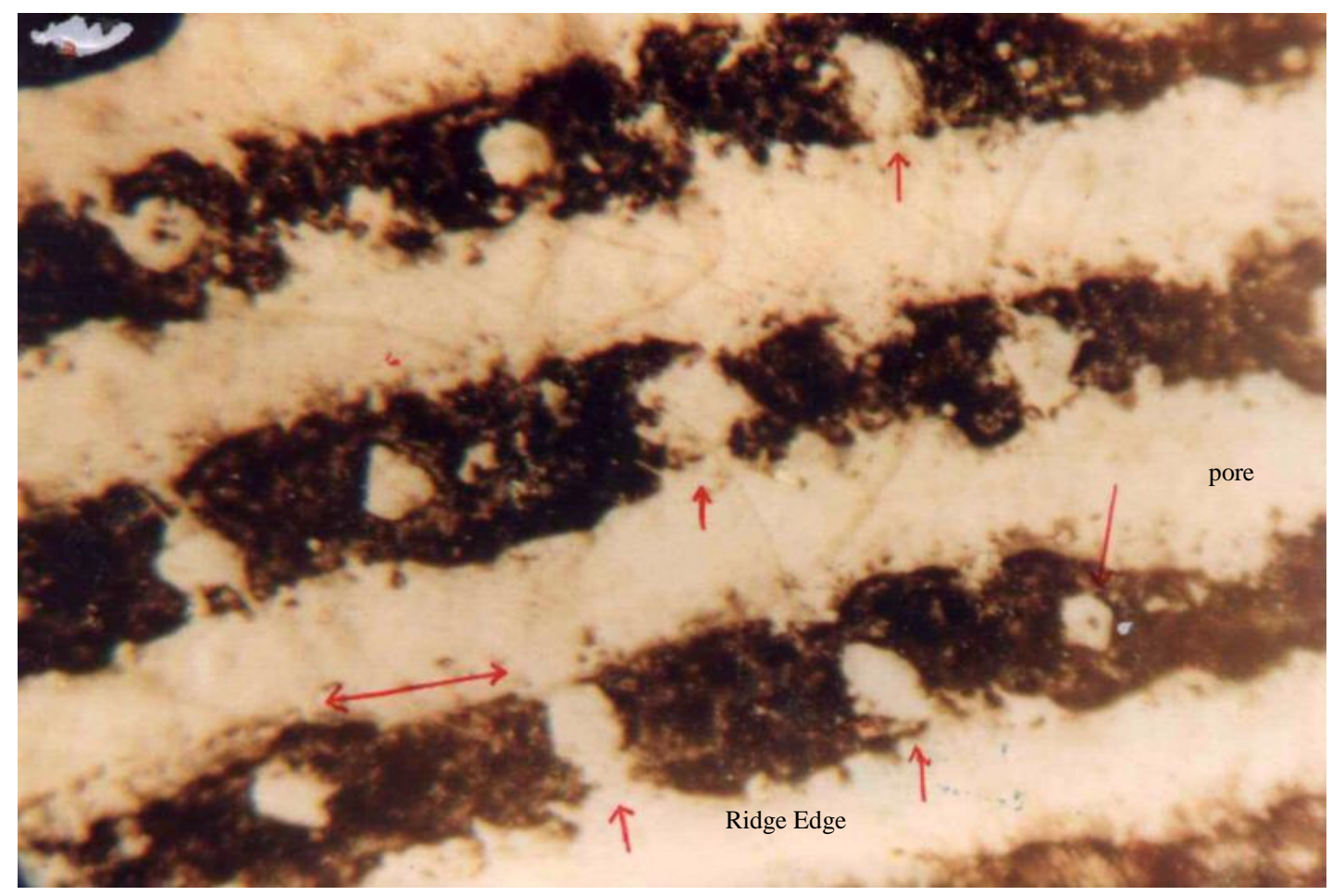

Fig 3-5 Pore and ridge edge contour

\subsection{Minutiae-Based Fingerprint Recognition}

Minutiae-based fingerprint representation [75] to design the systems due to the advantages of wide accessibility and stability has been described in Section 3.1.3. Minutiae-based fingerprint representation and matching are widely used by both machine and human experts. Minutiae representation has several advantages compared to other fingerprint representations (Section 3.1.3). Minutiae have been (historically) used as key features in fingerprint recognition tasks. Its configuration is highly distinctive and several theoretical models $[13,14$, and 15] use it to provide an approximation of the individuality of fingerprints. Minutiaebased systems are more accurate than correlation based systems [16] and the template size of minutiae-based fingerprint representation is small. Forensic experts use this representation which has now become part of several standards [12, 17] for exchange of information between different systems across the world. 


\subsection{Fingerprint Image Enhancement}

Fingerprint image quality is an important factor in the performance of minutiae extraction and matching algorithms. A good quality fingerprint image (Fig 3-6(a)) [59] has high contrast between ridges and valleys. A poor (Fig 3-6(b)) [59] quality fingerprint image is low in contrast, noisy, broken, or smudgy, causing spurious and missing minutiae. Poor quality can be due to cuts, creases, or bruises on the surface of finger tip, excessively wet or dry skin condition, uncooperative attitude of subjects, damaged and unclean scanner devices, low quality fingers (elderly people, manual workers), and other factors.

The goal of an enhancement algorithm is to improve the clarity (contrast) of the ridge structures in a fingerprint. General-purpose image enhancement techniques are not very useful due to the non-stationary nature of a fingerprint image. However, techniques such as gray-level smoothing, contrast stretching, histogram equalization, and Wiener filtering [18, 19, and 20] can be used as preprocessing steps before a sophisticated fingerprint enhancement algorithm is applied.

Techniques that use single filter convolutions on the entire image are not suitable. Usually, a fingerprint image is divided into sub regions and then a filter whose parameters are pre-tuned according to the region's characteristics is applied. Each local region of a fingerprint can be seen as a surface wave of a particular wave (ridge) orientation (perpendicular to the flow direction) and frequency. Several types of contextual filters in both spatial and frequency domains have been proposed in the literature [67]. The purpose of the filters is to fill small gaps (low-pass effect) in the direction of a ridge and to increase the discrimination (band-pass effect) between ridges and valleys in the direction orthogonal to the ridge [68]. O'Gorman and Nikerson [67] were the first to propose the use of contextual filtering. 


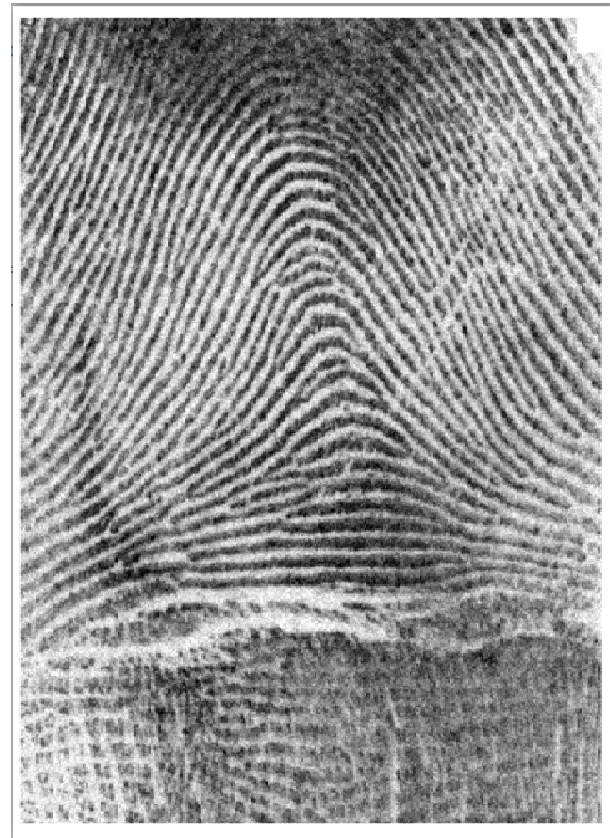

(a)

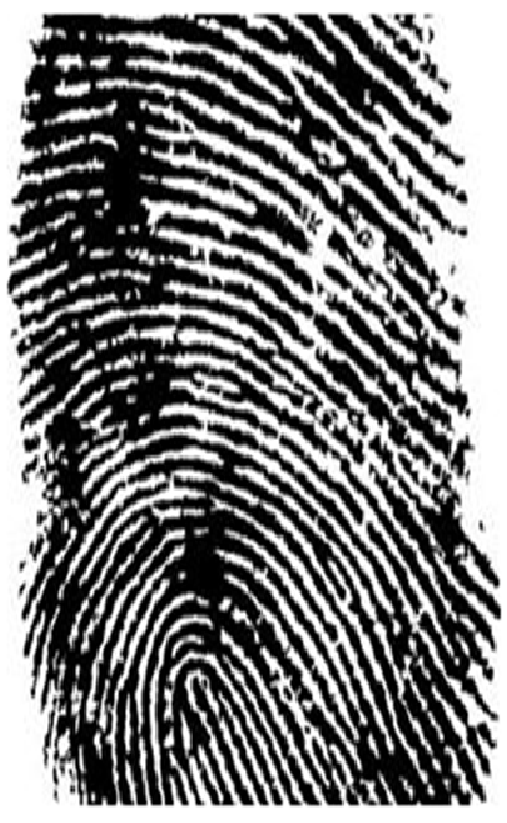

(b)

Fig 3-6 a) Good quality fingerprint image (b) Poor quality fingerprint image [59]

Recently, Greenberg et al [20] proposed the use of an anisotropic filter that adapts its parameters to the structure of the underlying sub region. Wu, Shi, and Govindaraju [21] proposed to convolve a fingerprint image with an anisotropic filter to remove Gaussian noise and then apply directional median filters (DMF) [80] to remove impulse noise.

On visual inspection, enhancement results of $\mathrm{Wu}$ et al [21] appear to be superior to those obtained by Greenberg et al [20].

Anisotropic filters remove Gaussian noise and smoothen the fingerprint image along the local ridge direction.

The standard rectangular-shaped median filters produce artifacts in fingerprint images. Wu et al [21] use directional median filters whose shapes vary by their direction. 
Note that the shape of the filter changes along with its direction.

Sherlock, Monro, and Millard [2] proposed a fingerprint enhancement method in the Fourier domain. In this approach, a fingerprint image is convolved with pre-computed filters which results in a set of filtered images. The enhanced fingerprint image is constructed by selecting each pixel from the filtered image whose orientation is the closest to that of the original pixel. However, their assumption of constant ridge frequency limits the performance of the approach. Willis and Myers [23] presented an FFT [9] based fingerprint enhancement method. Instead of explicitly computing the local ridge direction and frequency, enhancement is achieved by multiplying the Fourier transforms of the block by magnitude of power, $\mathrm{k}$ (1.4 in [23]). Chikkerur [24] proposed an algorithm based on short time Fourier transform (STFT), and a probabilistic approximation of dominant ridge orientation and frequency was used instead of the maximum response of the Fourier spectrum to remove impulse noise (small gaps on bridge or dots in valleys). The ridge orientation image, ridge frequency image, and foreground region image are generated simultaneously while performing the STFT analysis.

A wavelet-based method is proposed by Hsieh et al [25]. It uses both local ridge orientation and global texture information. Fingerprint image is first wavelet-decomposed into "approximation" and "detail" sub-images. A series of texture filters and a directional compensation process based on a voting technique are applied on those sub-images. The enhanced fingerprint image is then obtained by the reconstructing process of wavelet transform.

\subsection{Minutiae Extraction}

The reliability of minutiae features (see Section 3.1.3) plays a key role in automatic fingerprint recognition. Generally, the minutiae representation of a fingerprint consists of simply a list of minutia points associated with their spatial coordinates and orientation. Some methods also include the types [26, 27, 28, and 29] and quality [28, 30, and 31] of minutiae in the 
representation. Minutiae extraction algorithms are of two types: [59] (i) binarization-based extraction and (ii) gray-scale based extraction.

\subsubsection{Binarization-based Minutiae Extraction}

Most of the proposed minutiae extraction methods are binarization-based approaches. They require conversion of the gray-scale fingerprint image ( 8 bits per pixel, 256 gray levels) into a binary form (1 bit per pixel, black or white). Various binarization techniques have been presented in the image processing literature [33,32]. One intuitive approach is to use a global threshold (th) and assign each pixel a value as follows:

$$
\operatorname{IB}(x, y)=\left\{\begin{array}{l}
1 \text { if I }(x, y)>\text { th } \\
0 \text { if I }(x, y)<=\text { th }
\end{array}\right.
$$

where $\mathrm{I}(\mathrm{x}, \mathrm{y})$ is the intensity value of the pixel at $(\mathrm{x}, \mathrm{y})$ in a gray-scale image. Otsu's method [35] describes a technique to obtain the global threshold (th) from a statistical viewpoint. Dong and Yu [34] use a data clustering approach which is equivalent to Otsu's method [35] but is more efficient. The contrast variation in a fingerprint image makes it impossible to find an optimal global threshold. Adaptive techniques are preferred in general but they fail on poor quality images.

Several methods have been proposed to utilize the flow texture of a fingerprint image in binarization tasks. Stock and Swonger [36] observed that the average local intensity of a ridge line along its flow direction is highest and used it in binarization. Ratha, Chen and Jain [37] use a $16 \times 16$ window centered and oriented along the local ridge direction on each pixel. Ridge lines are recognized as peaks of the gray-level profile of pixel intensities projected on the central segment of the window. Coetzee and Botha [38] use a local binarization technique. The area between two edges of a local block is blobcolored and then logical ORed with the result of local binarization of the same local block to 
produce the final binarized image. Garris et al [30] and Watson et al [31] propose a directional binarization technique. In this approach, each pixel is examined successively and assigned to black (0) or white (1). By consulting the intrinsic orientation map, a pixel is assigned to white if there is no detectable ridge flow for the local block. If the flow is well defined in the pixel's local block, then an orientated window $(7 \times 9)$ is used to analyze the neighboring pixel intensity of the pixel (Fig 3-7). The rows of the window are aligned with the local ridge and the central row sum is compared against the average row sum of the entire window. A pixel is white if the central row sum is less than the window's average row sum; otherwise, it is black. Other fingerprint binarization methods can be found in [39, 40, 41, 42, 43, and 44].

Usually, the binarization-based minutiae extraction methods apply a thinning algorithm after the binarization step to obtain the skeletons of fingerprint ridges. Once a binary skeleton of a fingerprint is obtained, minutiae extraction becomes a trivial task. It is assumed that the foreground and background pixel values of a fingerprint skeleton are 1 and 0 , respectively. 


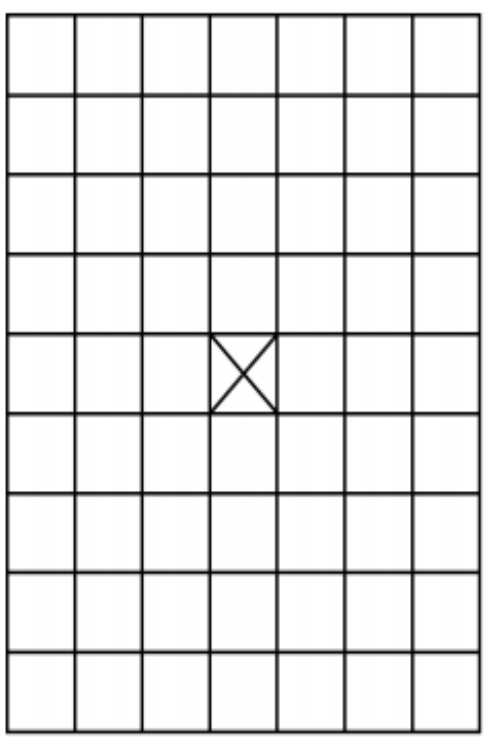

(a)

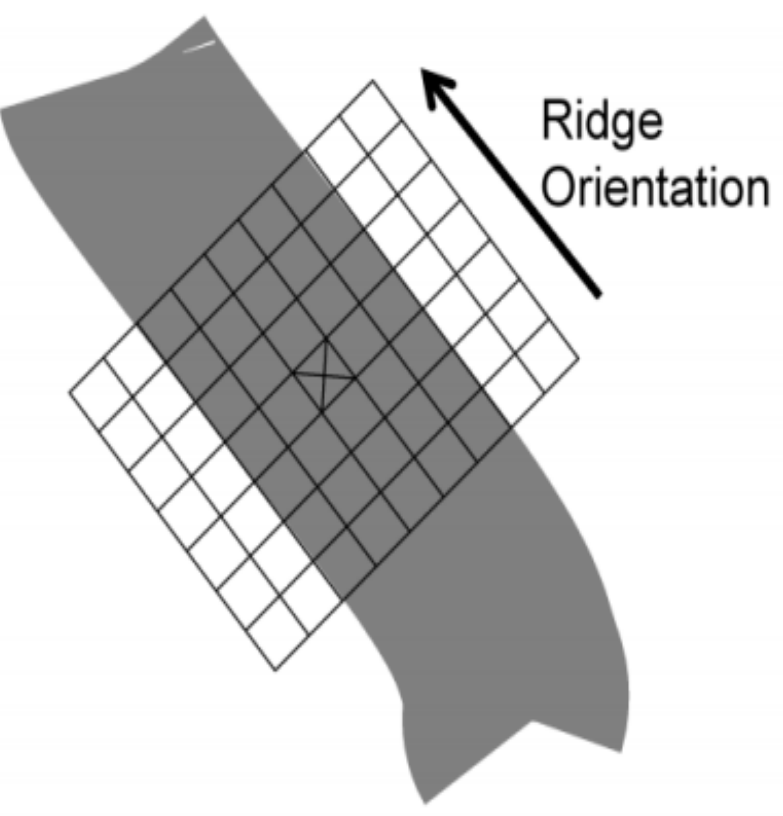

(b)

Fig 3-7 (a) The window used for analyzing the surrounding pixel intensity (b) the window oriented along the local ridge direction [59]

Minutia can be detected by examining the 8-neighborhood of a ridge skeleton pixel at $(x, y)$.

Many thinning approaches [86] have been proposed. However, thinning tends to introduce hair-like artifacts along the one-pixel wide skeleton, which leads to detection of spurious minutiae. Various techniques $[37,45]$ are introduced between the stages of binarization and thinning to improve the quality of binarized fingerprint images by filling holes, smoothing ridges, and removing small gaps and other artifacts.

Several approaches have been also proposed to extract minutiae directly from the binarized fingerprint image to avoid the computationally intensive thinning process. Weber [101] proposed a method that extracts minutiae from the thick binary ridges using 
a rule based ridge tracking algorithm. Garris et al [28] (see also Watson [100]) use a series of pixel patterns to detect minutiae on binraized fingerprint images. A method based on chaincode is proposed by Govindaraju et al [97]. It is a lossless representation of an object contour and is widely used in document analysis and recognition research [67]. It is generated by tracing the exterior contours of a binary object counterclockwise (clockwise for interior contours) and stored in contour lists. In the contour list, each contour element contains the $\mathrm{x}, \mathrm{y}$ coordinates of the pixel, the direction of the contour into the pixel, and curvature information. The chaincode representation of fingerprint ridge contours provides several advantages in minutia detection:

- It is a lossless representation, thus, most of fingerprint information is retained.

- It is easy to remove small objects and holes from the ridge contours. Therefore, the number of spurious minutiae is few.

- It works directly on binarized image and eliminates the need for thinning.

- Minutiae are the significant turns in the ridge contour. 


\section{CHAPTER 4}

\section{REVIEW OF THE AUTOMATIC FINGERPRINT IDENTIFICATION SYSTEM}

The following sections describe the components and algorithms that make up a typical fingerprint recognition system. While individual systems will not necessarily do things the way they are described here, the basic principles are described and examples are given wherever possible. Note that this is not always possible, due to the proprietary nature of the technology and algorithms involved. Also, there are two main system methodologies for fingerprint matching, those that utilize minutiae matching, and those that use pattern matching on the overall ridge structure.

\section{$\underline{4.1 \text { Capture Devices }}$}

The following sections describe the function of each component of a fingerprint capture device.

\subsubsection{Scanning}

For fingerprints there are a number of alternative data capture devices (Fig 4-1). These include optical scanners, silicon based capacitance and thermal sensors, pressure based sensor and ultrasound devices. The main objective of a fingerprint scanner, regardless of the method it uses, is to provide the system with an image of the fingerprint that is as accurate as possible. For most applications, the image is produced at a resolution of $500 \mathrm{dpi}$ using an 8-bit grayscale. The following sections describe how each of the different kinds of fingerprint scanners works. 


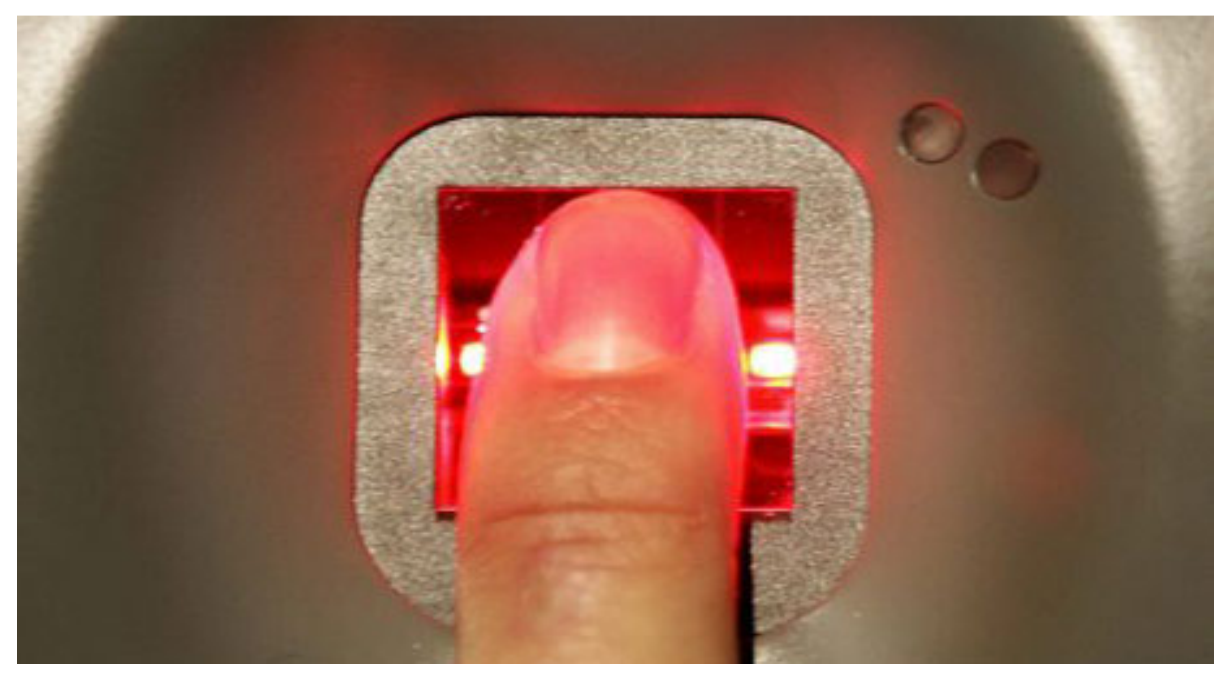

Fig 4-1 Fingerprint scanner

\subsubsection{Optical}

Optical devices $[22,54]$ are one of the more common fingerprint scanning devices. They are based on the reflection changes that occur when a light source interacts with the ridge lines of a fingerprint. This is most commonly achieved through the use of Frustrated Total Internal Reflection (FTIR). The light source shines onto a special reflection surface, which reflects the light differently depending on the pressure applied to it. A light sensor is used to capture the 'image' of the fingerprint. Fig 4-2 depicts the general layout of a typical optical scanner. Due to the involvement of pressure, this type of scanner returns different quality images depending on the pressure applied to the reflective surface (by the fingerprint).

If too little pressure is applied, the sensors may not be able to create an image at all. Alternatively, if too much pressure is applied, the skin in between the fingerprint ridges will also be in contact with the reflective surface, causing a lack of definition between ridges and valleys. Optical scanners are also affected by dirty fingerprints, which can also result in unusable images. 
Due to the required placements of the light source, reflective surface and light sensors, optical scanners are typically physically large components. However some companies have developed improved methods to reduce the size of the component. An example of this is the Surface Enhanced Irregular Reflection (SEIR) [81] technology patented by SecuGen Corporation. Another weakness of optical scanners is that they must be cleaned regularly, to avoid dust; dirt and oil build up on the reflective surface. Also, optical scanners are the most vulnerable to physical replay attacks. This is where the latent print of the previous user is used to gain access to the system. This can theoretically be achieved in some scanners simply by shining an external light at the correct angle.

Optical models generally claim that they cannot be fooled by a 2-dimensional image of a fingerprint; however a simple 3D model (e.g. latex rubber or similar product) is often sufficient.

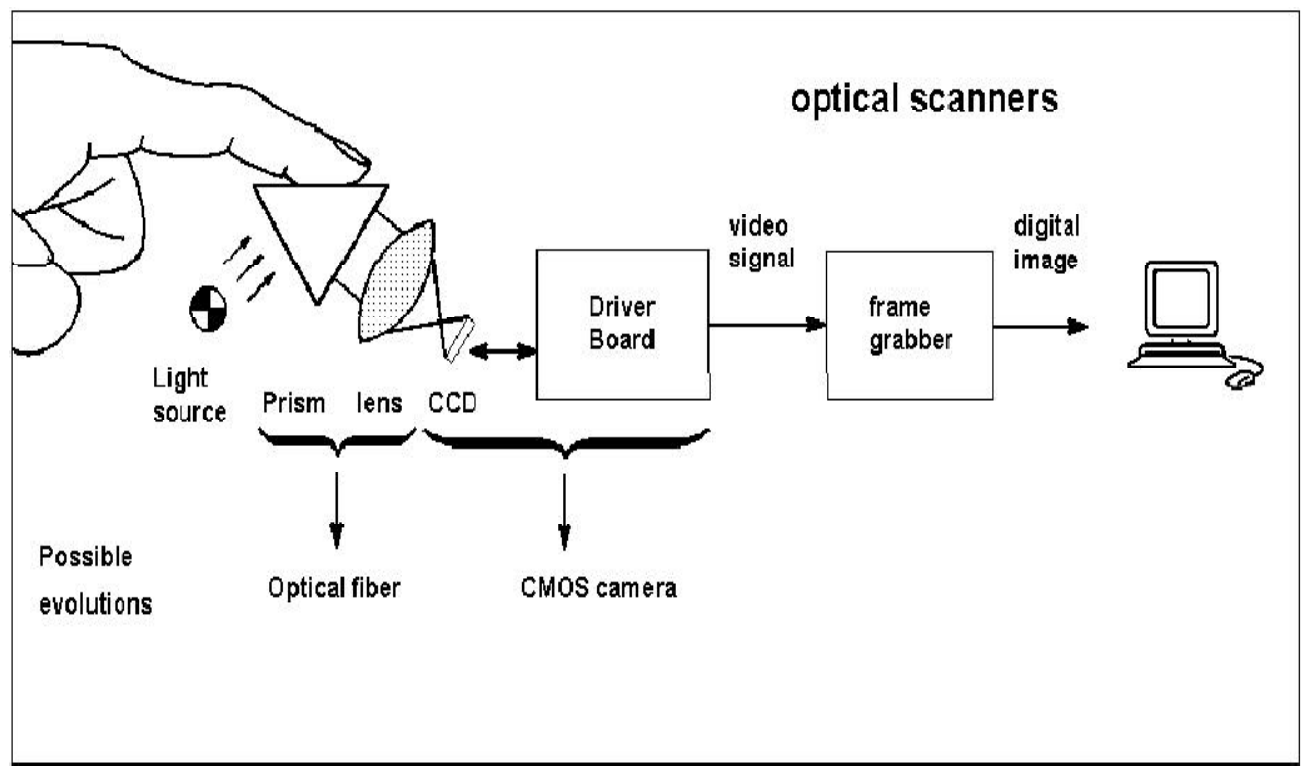

Fig 4-2 General layout of an optical fingerprint scanner, reproduced from [Atmel Corporation 2001] [54] 


\subsubsection{Capacitance}

Capacitive fingerprint scanners $[22,54]$ create images of a fingerprint through the use of rectangular arrays of capacitors. The capacitors are located under a very thin protective layer that must be thick enough to protect the capacitors, but also thin enough so it does not obscure the readings. When a finger is placed on the scanner, the capacitors measure the capacitance difference generated by the different distances between the capacitors and the ridge lines and furrows. This concept is depicted in Fig 4-3. One disadvantage of using this method is that the capacitance difference is significantly affected by moisture. Hence, fingers that are too wet, oily or dry will generate low quality or unusable images. Also, the capacitors themselves are vulnerable to Electro-Static Discharge (ESD) and external electric fields. As a result the grounding of capacitance scanners is very important to prevent damage to the capacitors. One major advantage of capacitance scanners is their reduced size. Scanners using this method have been produced small enough to fit on PCM/CIA cards, and hence are convenient for use in small portable devices such as laptop computers and mobile phones.

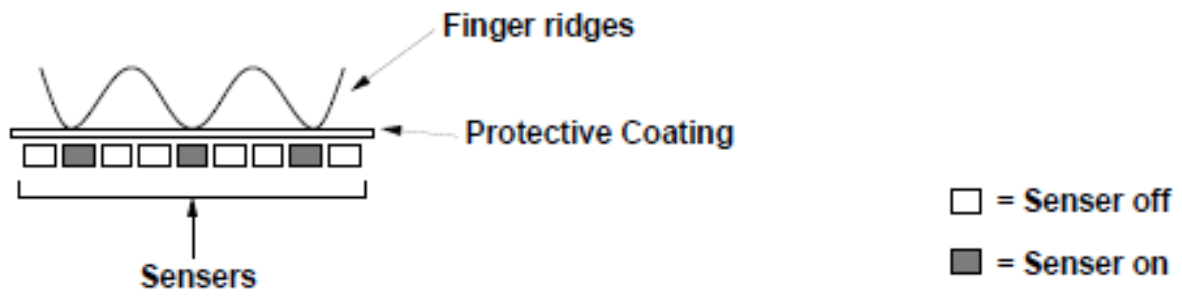

Fig 4-3 Depiction of a capacitance scanner [54]

\subsubsection{Thermal}

Thermal fingerprint scanners $[22,54]$ work in a manner similar to capacitance scanners. However, instead of measuring the difference in temperature between the ridges and valleys of the fingerprint (which are too small to measure effectively), the fingerprint image is generated by converting the temperature differential on a sensor. For example, before a fingerprint is placed 
on the scanner, all the thermal sensors are at equilibrium with the air temperature around the scanner. Then when a fingerprint is placed on the sensor, those points where a ridge is in contact will cause a change in temperature in the sensor for that point. However, those points where there is a valley will still be measuring the local air temperature; hence those points have no temperature differential, and are not activated.

The major drawback of this method is that the temperature difference disappears after less than a tenth of a second as the finger and the sensors reach equilibrium. As a result, the image is only available for a short period of time. This means that a user has a very short amount of time to position his/her finger appropriately, before the image is gone. In order to counter this, Atmel Corporation, a major producer of thermal fingerprint scanners has developed and patented a sweeping technique. This technique works by sweeping a finger over the sensor array, which takes the images from different times over the sweep (in a manner that is not affected by the sweeping speed) and reconstructs the total fingerprint image from these images using a proprietary and private algorithm. This allows the scanner to be reduced to approximately $1 / 5$ the size of a normal square array scanner and eliminates the problem of the temperature equalization, as the sweeping motion ensures that any one sensor is constantly changing between finger temperature (ridge) and air temperature (valley).

\subsubsection{Pressure}

Purely pressure-based scanners $[22,54]$ that utilize silicon chips to convert pressure to an electrical signal have been developed. Due to the natural pressure that is applied when a finger is placed on a scanner, this is probably the most intuitive method to use. However, as these scanners generally have low sensitivity, which is further lowered when a protective layer is added; the resulting images have quite low detail. As a result, no known systems utilize this method. 


\subsubsection{Ultrasound}

Ultrasound scanners $[22,54]$ are the least common commercial option for fingerprint scanners. They operate by using acoustic energy that is partially reflected at each interface between different materials. The time between the reflections can be accurately measured to determine the depth at which the reflection occurred (see Fig 4-4). The advantage of using ultrasound is that the images returned are uncontaminated by any dirt or grease on the surface on which the finger rests, or on the finger itself (from [Ultra-Scan Corporation]). This, according to the manufacturer, results in higher quality images than is possible with optical systems. It also renders a physical replay attack (using a latent print) conceptually impossible as a latent print should not reflect sounds waves.

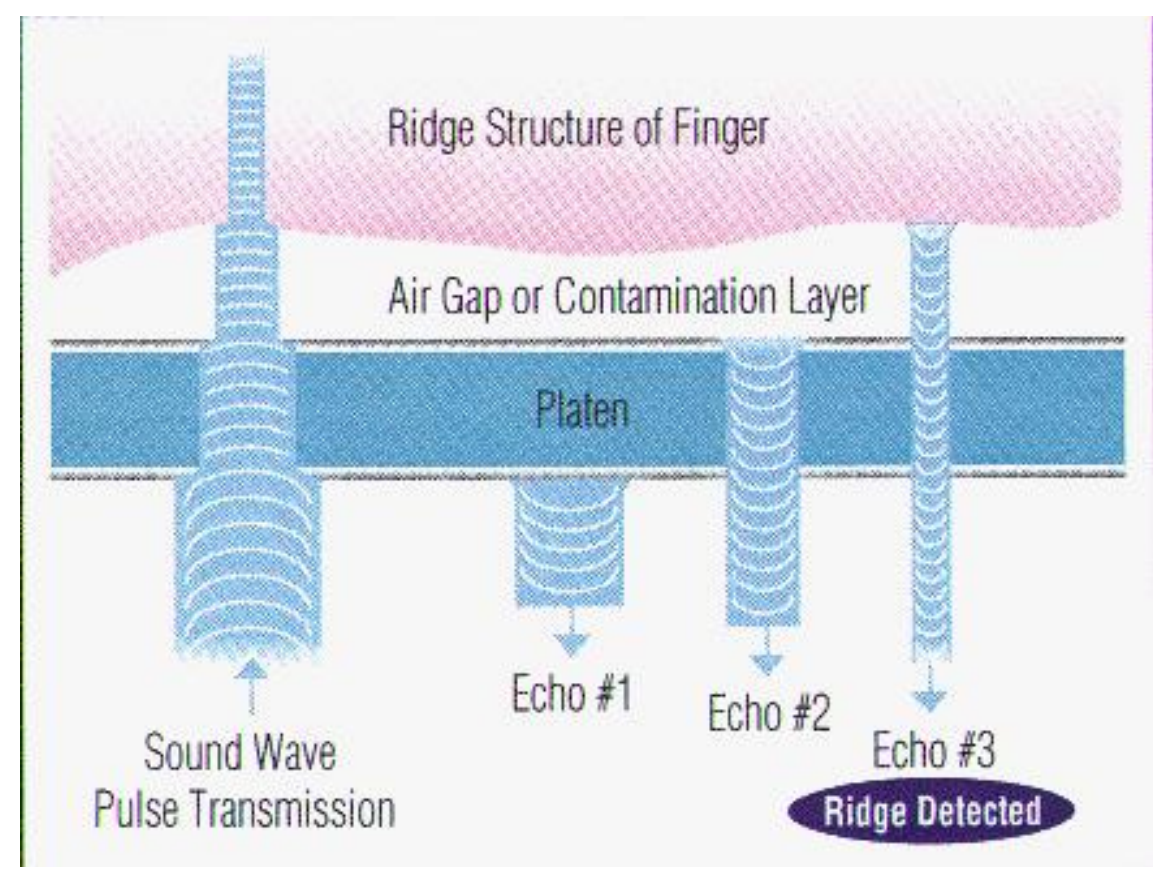

Fig 4-4 Operation of ultrasound scanner, sound waves return a partial echo at each change in material, from Ultra-Scan [54] 
However, despite these advantages, ultrasound scanners have a number of major drawbacks. Firstly, they are a large device (approximately $15 \times 15 \times 20 \mathrm{~cm}$ ), preventing integration into small or portable components. In addition they are noisy (due to internal mechanical components), expensive and slow, with a single scan (at the highest quality setting) taking up to 4.60 seconds, according to [Ultra-Scan Corporation].

\section{$\underline{4.2 \text { Scanning a Fingerprint }}$}

Many of the algorithms require a linear scan of the fingerprint image. Scanning is achieved by moving a fixed size window across the picture in a grid-like pattern. This can be seen in the Fig 4-5. However, it is possible that areas of interest do not lie squarely in the one of the windows. To account for this the window is then shifted just vertically, just horizontally, and then vertically and horizontally by half the window size and the grid scan is completed again. Therefore, it takes four scans of the image to do the linear scan. This is not a problem because it is used for the preprocessing of a fingerprint image (which occurs only once) and is done in a linear manner.

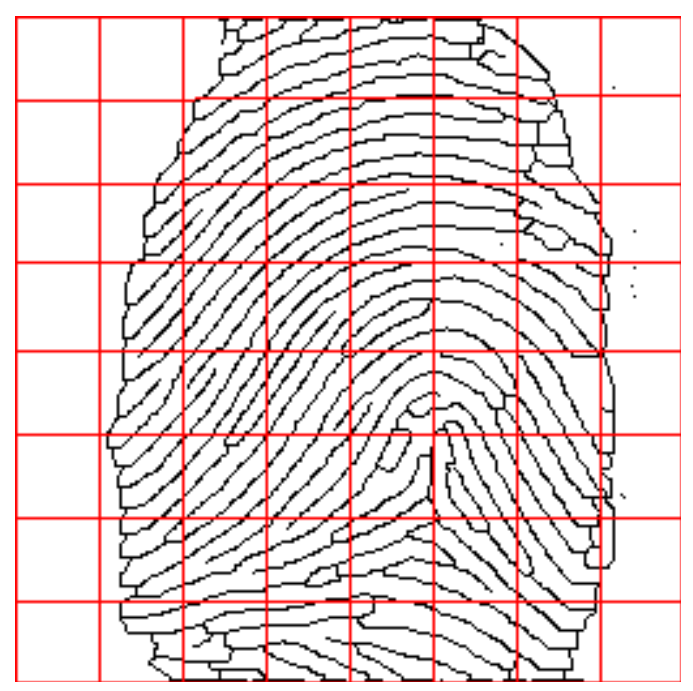

Fig 4-5 Scanned image 


\section{$\underline{4.3 \text { Steps for fingerprint enhancement }}$}

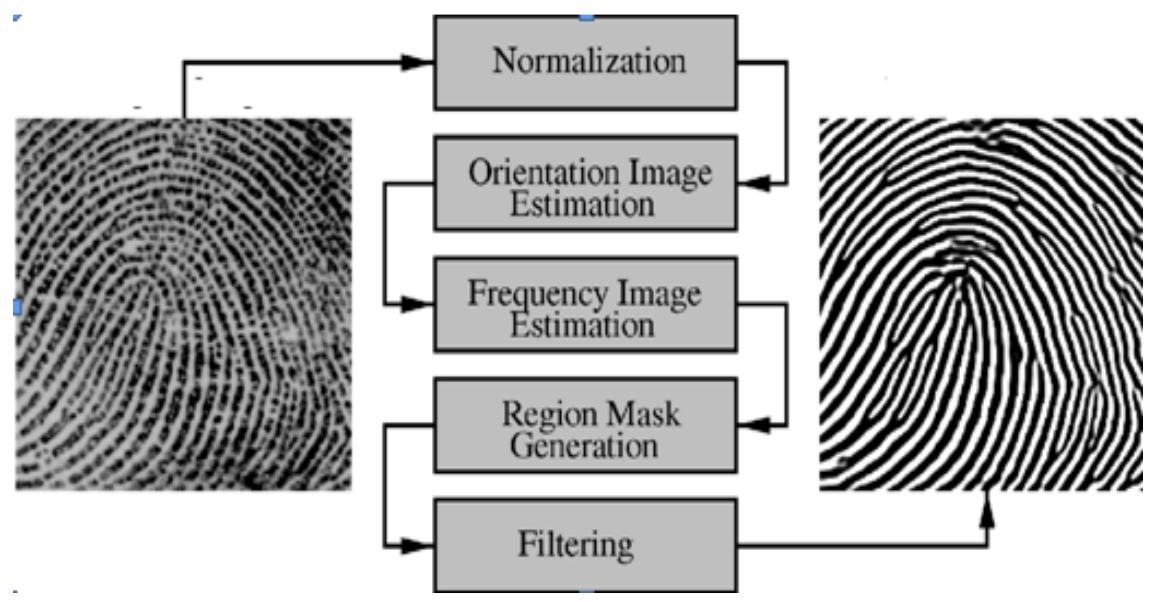

Fig 4-6 A flowchart of the proposed fingerprint enhancement algorithm [3]

The flowchart of the fingerprint enhancement algorithm is shown in Fig 4-6[3]

1) The image is first normalized to have desired mean and variance

2) It is then divided into non-overlapping blocks

3) Dominant ridge orientation is determined for each block

4) They are then smoothed and subsequently the block direction image is formed

5) Average ridge distance for the whole input image is determined

6) Directional filtering is used to enhance the image (Fig 4-6)

\section{$\underline{4.4 \text { Normalization }}$}

Normalization is used to standardize the intensity values in an image by adjusting the range of grey-level values so that it lies within a desired range of values.

The mean and variance of a gray level fingerprint image I are: 


$$
\begin{gathered}
M(I)=\frac{1}{N^{2}} \sum_{i=0}^{N-1} \sum_{j=0}^{N-1} I(i, j) \\
\operatorname{VAR}(I)=\frac{1}{N^{2}} \sum_{i=0}^{N-1} \sum_{j=0}^{N-1}(I(i, j)-M(I))^{2}
\end{gathered}
$$

Let $N(i ; \jmath)$ represent the normalized grey-level value at pixel $(i, j)$. The normalized image is defined as

$$
M_{0}+\sqrt{\frac{V A R_{0}(I(i, j)-M(I))^{2}}{V A R}} \quad \text { if } I(i, j)>\mathrm{M}
$$

$N(\mathrm{i}, \mathrm{j})=$

$$
M_{0}-\sqrt{\frac{V A R_{0}(I(i, j)-M(I))^{2}}{V A R}} \quad \text { otherwise }
$$

where $\mathrm{M}_{0}$ and $V A R_{0}$ are the desired mean and variance respectively. Normalization is pixel-wise operation. It does not change the clarity of the ridge and furrow structures.

The main purpose of normalization is (Fig 4-7)

1) To have images with similar characteristics

2) To remove the effect of the sensor noise.

3) To reduce the variation in gray level values along ridges and valleys. 


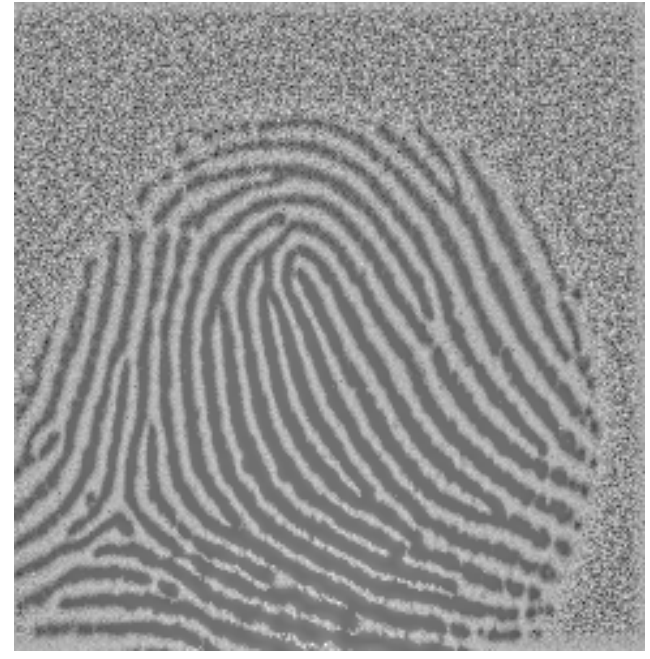

Fig 4-7 Normalized image [7]

\subsection{LRO (Local Ridge Orientation) (Fig 4-8):}

The orientation field $O$ is defined as a $P x Q$ image where $O(i, j)$ represents the local ridge orientation at pixel(i,j).[1]

There are a number of techniques to calculate orientation fields; Local mean square estimation based on gradient has been used.

1. The input image is first divided into a number of non-overlapping blocks

2. For each pixel $p$ of the block the $x$ and $y$ components of the gradient, Gx and Gy respectively, are calculated.

$$
G x=D p / D x
$$

$$
G y=\triangleright p / \triangleright y
$$

$\left[\begin{array}{ll}G_{s, x} & G_{s, y}\end{array}\right]^{T}=\left[\sum_{w}\left(G_{x}^{2}-G_{y}^{2}\right) \quad \sum_{w} 2 G_{x} G_{y}\right]^{T}$ 
3. The average gradient op direction and dominant local orientation for the block are given by

$$
\begin{gathered}
\frac{1}{2} \tan ^{-1} \frac{\sum_{\mathrm{w}} 2 \mathrm{G}_{\mathrm{x}} \mathrm{G}_{\mathrm{y}}}{\sum_{\mathrm{w}}\left(\mathrm{G}_{\mathrm{x}}^{2}-\mathrm{G}_{\mathrm{y}}^{2}\right)} \\
\mathrm{O}(\mathrm{i}, \mathrm{j})=\phi+\Pi / 2
\end{gathered}
$$

Additional low pass filtering is done in order to eliminate the wrongly estimated ridge.

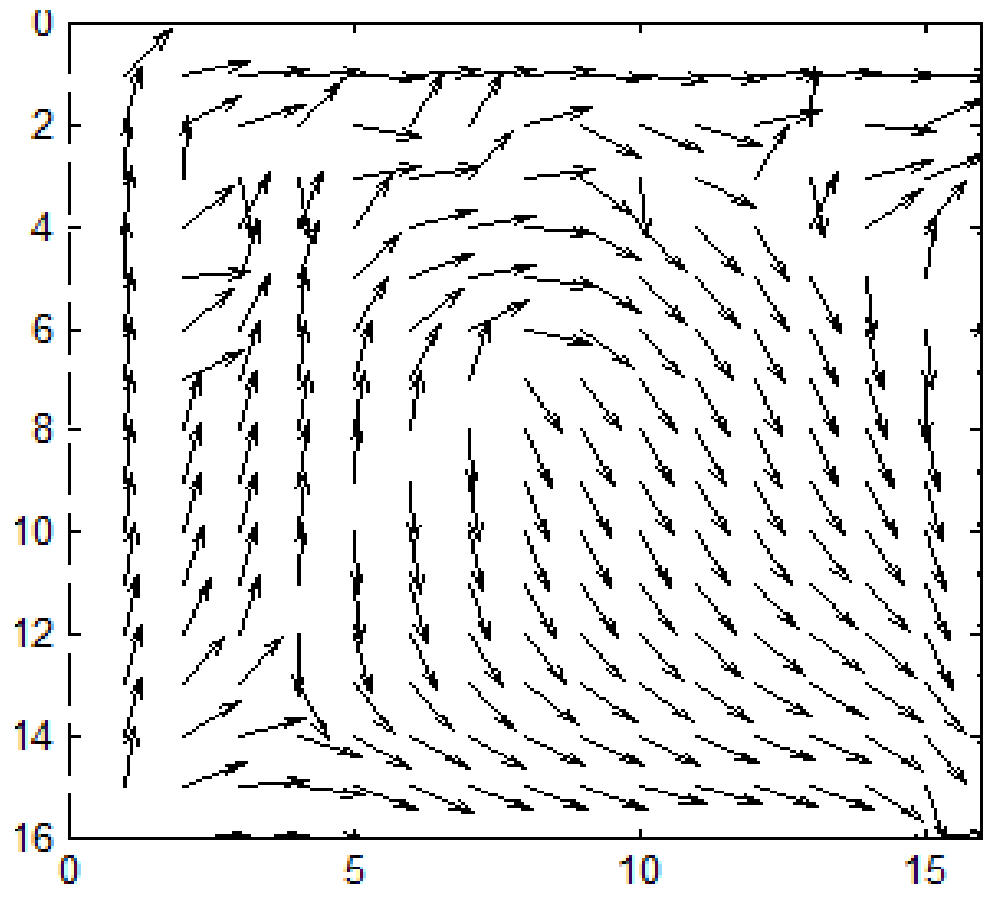

Fig 4-8 Orientation field image 


\subsection{LRF (Local Ridge Frequency) [1]}

1) Project gray values of all the pixels located in each block along a direction orthogonal to the local orientation computed above. The projection forms 1D wave with the local extrema corresponding to the ridges and valleys.

2) $L(i, j)$ is the average number of pixels between two consecutive peaks in 1D wave. The frequency $f(i, j)$ is calculated as:

$$
f(i, j)=1 / L(i, j)
$$

\subsection{Algorithm for fingerprint enhancement: [1], [3]}

1) The fast Fourier transform (FFT) (Fig 4-9) [9] of the fingerprint image is computed. Fast Fourier transform is a discrete Fourier transform algorithm which reduces the number of computations [Fig. 4.9] needed for $\mathrm{N}$ points from $2 \mathrm{~N}^{2}$ to $2 \mathrm{Nlog}_{2} \mathrm{~N}$.

2) Fourier transform of the filter components $P_{i}$ depends upon the orientation values. Each directional filter $P_{i}$ is point by point multiplied by $F$, obtaining $n$ filtered image transforms $P F_{i}, i=1,2, \ldots \ldots, n$.

3) Inverse FFT is calculated for each $P F_{i}$ resulting in $n$ filtered images $I_{i}, i=1,2, \ldots ., n$ (spatial domain).Inverse FFT is calculated which is exactly the reverse process of FFT

4) The filtered image is then formed by selecting, for each pixel position, the pixel value from the pre-filtered image whose direction of filtering corresponds most closely to the actual ridge orientation at that position.

The sinusoidal shaped waves of ridges and valleys vary slowly in a local constant orientation. Therefore a bandpass filter tuned to the corresponding frequency and orientation can effectively remove the noise and preserve the true ridge and valley structures.

For directional filtering, Gabor filter is proposed: [1], [4] 


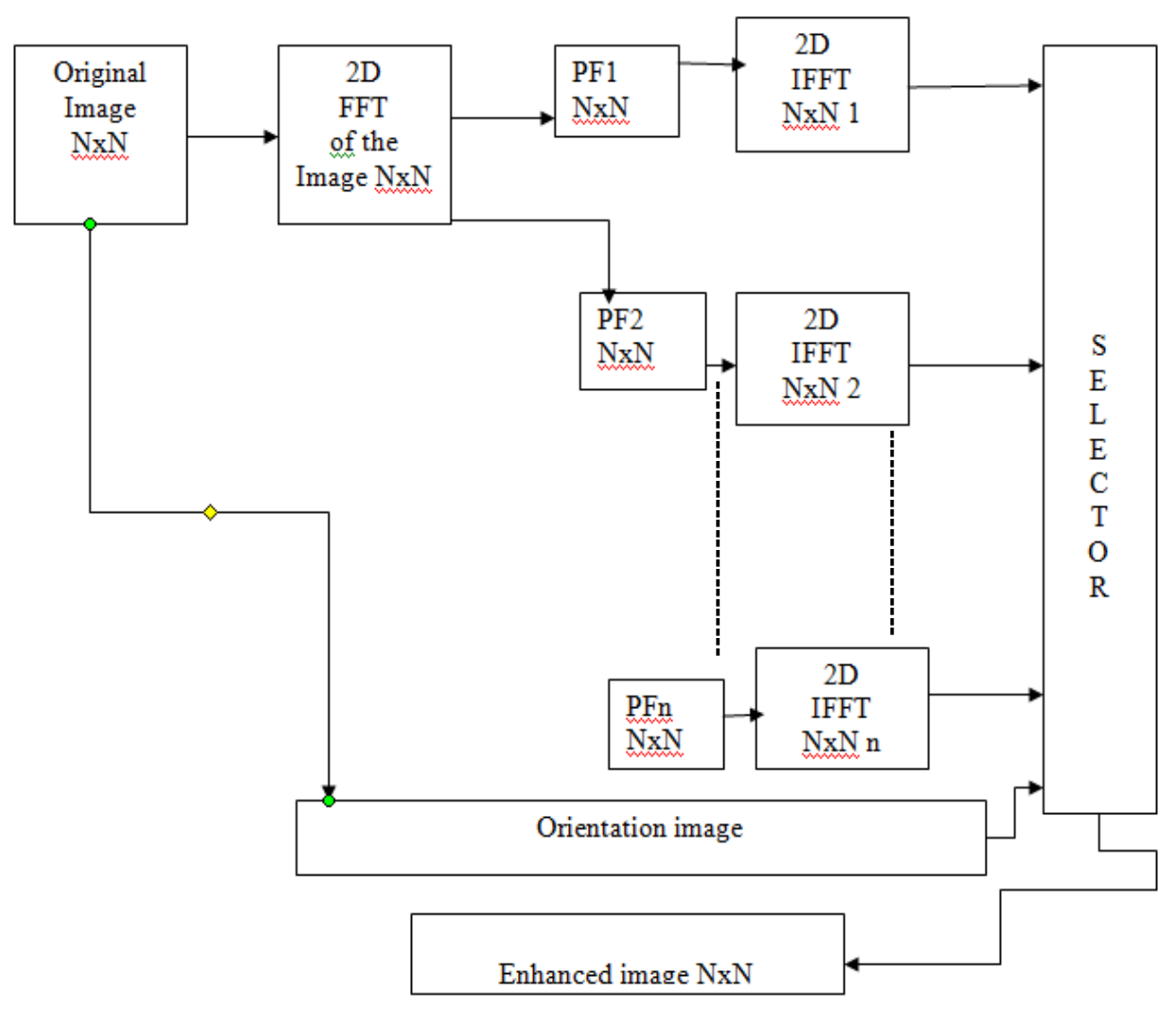

Fig 4-9 Algorithm for fingerprint enhancement [1] 
CHAPTER 5

FINGERPRINT IMAGE ENHANCEMENT

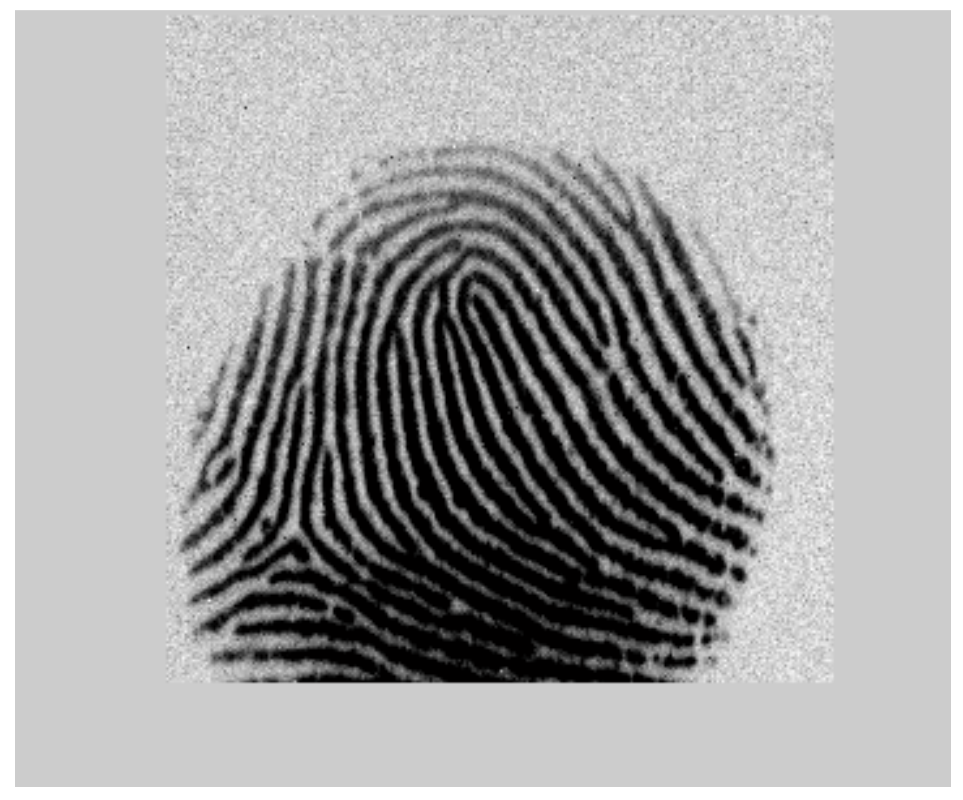

Fig 5-1 Original fingerprint [83]

\section{$\underline{5.1 \text { Image Enhancement }}$}

Original image is shown in Fig 5-1. Image enhancement [83] operation improves the quality of the image and it can be used to improve the image contrast and brightness characteristics, reduce its noise content, and/or sharpen its details. Image enhancement techniques may be grouped as either subjective enhancement or objective enhancement. Subjective enhancement technique may be repeatedly applied in various forms until the observer feels that the image yields the details necessary for particular application. Objective image enhancement corrects an image for known degradations. Here distortions are known and enhancement is not applied 
arbitrarily. This enhancement is not repeatedly applied but applied once based on the measurements taken from the system. Image enhancement falls into two broad categories: Spatial domain technique and Frequency domain technique. Spatial domain refers to the image plane itself, where approaches in this category are based on direct manipulation of pixels in an image. Also, spatial domain refers to the aggregate of pixels composing an image.

They operate directly on these pixels. Frequency domain processing techniques are based on modifying the Fourier transform of an image. The original image is processed and various stages are shown in Fig 5-2.

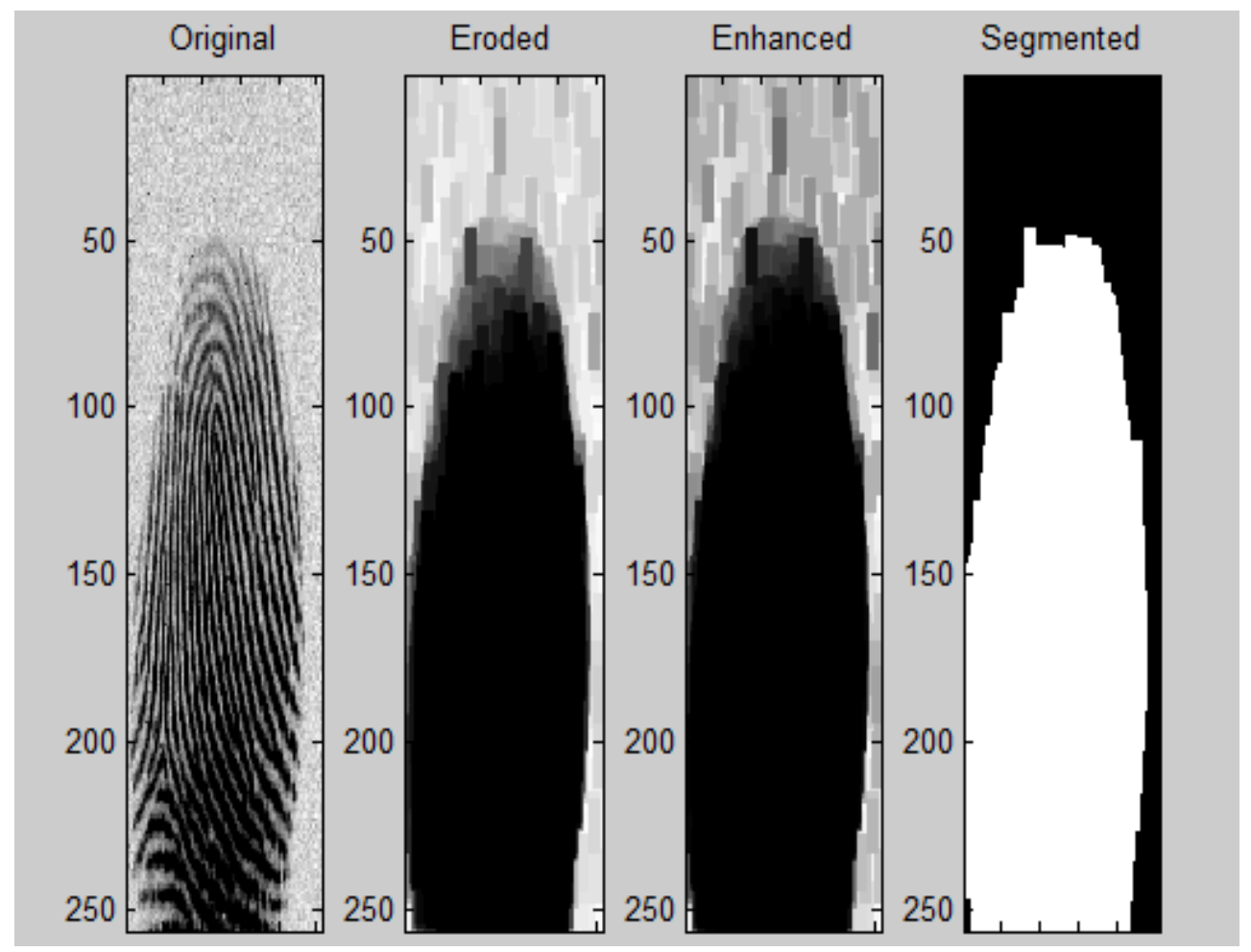

Fig 5-2 Various stages in the enhancement process 
Image enhancement means "making an image more vivid for human vision". Fingerprint recognition has been used in identifying individuals for a long time and it is the most popular method in biometric authentication at present. Consequently, no single standard method of enhancement can be said to be "the best". Besides that, the nature of each image in terms of distribution of pixel values will change from one area to another. Minutiae, typically including termination and bifurcation, are characteristic features of fingerprints that determine their uniqueness. In fingerprint recognition systems, valid minutiae can be hidden and spurious minutiae can be produced due to the low quality of fingerprint image. So, fingerprint enhancement is often required to enhance the quality of fingerprint image $[3,20]$. Contrast of an image is determined by its dynamic range, which is defined as a ratio between the brightest and the darkest pixel intensities. Contrast enhancement techniques have various application areas for enhancing visual quality of low contrast images $[72,73]$.

\subsubsection{Orientation Estimation and Verification}

Several methods of orientation estimation have been proposed including matched-filter based approach [16], high-frequency power method [67], and the simplest and most frequently adopted gradient-based approach [3, 4]. The associated orientations calculated by the gradientbased approach with different operators (Kirsch, Robinson, Sobel, and Prewitt [76]) are also shown in Fig 5-3. The top row of the Fig 5-3 shows six relatively clean ridge patterns (high contrast) where (a) and (b) contain minutiae (ridge bifurcation and ridge ending), (c) and (d) contain singular points (delta and core point), and the other two are noisy regions. Fig 5-3(e) shows a circle -like pattern with a white circle having a black dot inside, and (f) looks like a grid pattern with orthogonal ridge flows overlapping. It is observed that these four gradient operators generate relatively consistent block orientations. However, only the orientations of patterns (a) and (b) are reliable, and the other four are not. Actually, patterns (c) to (f) should not be assigned any orientation since no orientation can properly represent these ridge flows. 


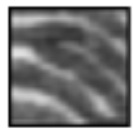

(a)

164.4 ë

163.5 ë

$161.3 \mathrm{e}$

160.6 ë

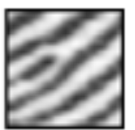

(b)

$29.5 \mathrm{e}$

$29.6 \mathrm{e}$

$31.4 \mathrm{e}$

$30.3 \mathrm{e}$

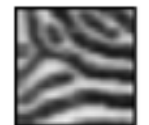

(c)

177.2 ë

176.5 ë

175.1 ë

175.2 ë

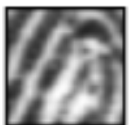

(d)

$64.4 \mathrm{e}$

$64.0 \mathrm{e}$

$62.1 \mathrm{e}$

$64.3 \mathrm{e}$

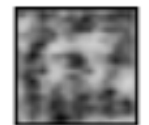

(e)

$42.9 \mathrm{e}$

$51.1 \mathrm{ë}$

49.3 ë

81.7 ë

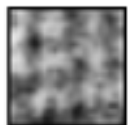

(f)

$62.4 \mathrm{e}$

63.0 ë

$60.1 \mathrm{e}$

69.3 ë

Fig 5-3 Six ridge patterns and their orientations calculated by Kirsch( $1^{\text {st }}$ row $)$, Robinson( $2^{\text {nd }}$ row $)$, Sobel( $\left(3^{\text {rd }}\right.$ row $)$, and Prewitt operators ( $4^{\text {th }}$ row) [77]

Ideally, the intensity value of the orientation orthogonal to ridge flows can be modeled as a sinusoidal plane wave (see Fig 5-4(a)). The width of the sinusoidal frequency can be considered as the ridge frequency. This approach works under an assumption that a reliable ridge orientation is already given. However, the previously mentioned algorithms will generate a ridge orientation no matter how the image quality is. A noisy region may lead to a wrong ridge orientation and a wrong ridge frequency. In such a case, applying an anisotropic filter on this noisy region can hardly improve the quality of the image but introduces more false ridges and features.

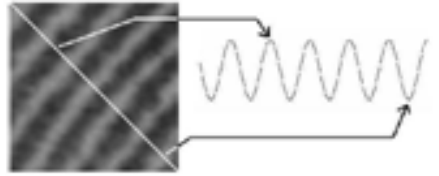

(a)

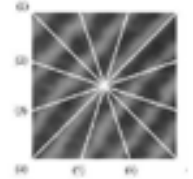

(b)

Fig 5-4 (a) A fingerprint ridge flows with ideal corresponding histogram. (b) Six directions for histograms examination [77].

A good orientation estimation algorithm should not only properly calculate flow orientation, but also prevent assigning an orientation to noisy blocks or blocks containing singular points. 


\subsubsection{Predicting ridge orientations using minutiae triplets}

Orientation of an image has been estimated in various ways. The orientation of a minutia is an indication of the local ridge direction since the fingerprint is a smoothly changing oriented texture pattern. Therefore, by observing the orientation of a group of minutiae one can 'interpolate' the underlying local ridge information. The proposed algorithm utilizes a set of three minutiae points (minutiae triplet) to predict the orientation of a triangular fingerprint region defined by the triplet. In the formulation, a minutia is represented as a three-tuple value, $(x, y$, $\theta)$, where $(x, y)$ is its spatial location and $\theta$ is its orientation. The algorithm [75] for generating the orientation map has four main stages: (i) triplet generation; (ii) orientation prediction; (iii) triplet pruning; and (iv) orientation smoothing.

1. Triplet generation:

Consider a minutiae template, $\mathrm{M}$, of a fingerprint containing $\mathrm{N}$ minutiae points given by, $M=\left\{m_{1}, m_{2}, \ldots . . m_{N}\right\}$ where $m_{i}=\left\{x_{i}, y_{i}, \theta_{i}\right\} . A$ set of 3 minutiae points $\left\{m_{i}\right\}_{i=1,2,3}$, characterized by a triangle with sides $\left\{L_{i}\right\}_{i=1,2,3}$ and interior angles $\left\{\Phi_{i}\right\}_{i=1,2,3}$

(a) $L_{\min } \leq L_{i} \leq L_{\max }$, for all $i=1,2,3$. This ensures that the perimeter of the triangle traverses a compact region, thus, avoiding the large global variability observed in the fingerprints of most classes.

(b) $\theta_{\text {dif }} \leq \theta_{\text {min }}$ where $\theta_{\text {dif }}=\max _{i=1,2,3}\left(\theta_{i}-\theta_{\text {med }}\right)$ and $\theta_{\text {med }}$ is the median of $\left\{\theta_{i}\right\}_{i=1,2,3}$. This ensures that the orientations of component minutiae points are within a small interval.

(c) $\theta_{i} \leq \theta_{\min }$, for all $i=1,2,3$. This ensures that "narrow" triangles subtending a very small area are avoided.

In the experiment performed [75] the values assumed are $L_{\max }=300, \theta_{\text {total }}=30^{0}$. 
2. Orientation prediction: Consider a pixel $P(x, y)$ located inside the triangular region defined by the triplet, $T$. Let $d_{i}=$ dist $\left\{m_{i}, P\right\}$, be the Euclidean distance of this pixel from all the three vertices such that $d_{1}<d_{2}<d_{3}$. The orientation of the pixel, $O(x, y)$, is then computed as,

$$
O(x, y)=\frac{d_{3}}{d_{1}+d_{2}+d_{3}} \theta_{1}+\frac{d_{2}}{d_{1}+d_{2}+d_{3}} \theta_{2}+\frac{d_{1}}{d_{1}+d_{2}+d_{3}} \theta_{3}
$$

The angle $\theta_{1}\left(\theta_{3}\right)$ corresponds to the orientation of the vertex that is nearest to (farthest from) the pixel $P(x, y)$. Thus, $O(x, y)$ is computed as a weighted sum of all the three orientations with a higher weight being assigned to the orientation of the closest vertex. The generated orientation map is shown in Fig 5-5. The orientations of ridges and, thus, of associated minutiae in whorls change rapidly over a small area (e.g. at the core region). Therefore, if triplets covering a large area are selected in the case of whorls, then an accurate prediction may not be rendered. However, in the case of arches, larger triplets provide good results since such rapid variations are not observed. The triplet generation procedure described above is more conservative and is observed to work well for all classes of fingerprints.

3. Triplet pruning: In a fingerprint image, minutiae tend to appear in clusters. For instance, the regions near the core and delta have dense minutiae activity. It is therefore possible for a triplet to reside inside the triangular region of another triplet or overlap with it. In such cases, rather than consolidating the orientation information predicted by multiple triplets, the information predicted only by a single good quality triplet is utilized. The quality, $Q$, of each selected triplet is measured by examining the average length of the sides of the triangle $\left(\mathrm{L}_{\mathrm{avg}}\right)$, the orientations of component minutiae points with respect to the median $\left(\theta_{\text {diff }}\right)$, and is computed as, 


$$
\mathrm{Q}=\left(\mathrm{L}_{\max }-\mathrm{L}_{\mathrm{avg}}\right) \omega_{1}+\left(\frac{\theta_{t o l}-\theta_{\text {dif }}}{\theta_{\text {dif }}} L_{\max }\right) \omega_{2}
$$

Here, $\omega_{1}$ and $\omega_{2}$ are the weights associated with each term $\omega_{1}=0: 4$ and $\omega_{2}=0: 6$, in the experiments). This ensures that a triplet having minutiae of similar orientations and traversing a relatively compact region is assigned a higher $Q$ value ( Fig 5-5 (b)). A good quality triangle will lead to a better estimation of the orientation of the underlying ridges according to the algorithm. The orientation at discrete points (every 13th pixel) in the fingerprint region is computed. Hence, for a $512 \times 512$ image, a $39 \times 39$ orientation map is generated.

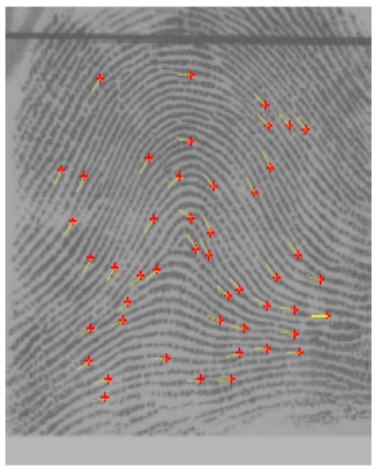

(a)

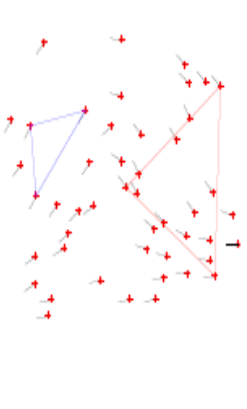

(b)

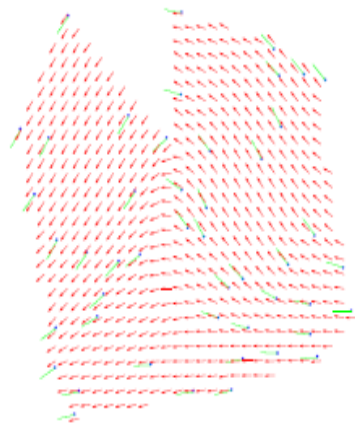

(c)

Fig 5-5 (a) Minutiae distribution of a fingerprint. (b) Examples of a good quality triplet (blue) with $\operatorname{Lavg}=112: 66, \theta$ diff $=5, Q=237: 63$ and a bad quality triplet (red) with $\operatorname{Lavg}=217$, diff $=26, Q=67: 55$. (c) Estimated orientation map[75]

4. Averaging the orientation map: To obtain a smooth transition in orientations, the predicted orientation map is convolved with a $3 \times 3$ local averaging filter.

\subsection{Orientation method used in this thesis}

The method used in this thesis for estimating the orientation of the image has been described in detail. 


\subsubsection{LRO (Local Ridge Orientation)}

The value of LRO at each pixel (i.e. the orientation image) is required as parametric input to the filter. Since determining LRO reliably can be computationally demanding, it may not be feasible to estimate LRO directly for every pixel. The approach is to determine LRO at a square grid spaced (say) 16 pixels apart, and obtain intermediate values by interpolation. An alternative, equally acceptable approach is to use a faster but perhaps less reliable algorithm to estimate orientation at every pixel position and to smooth the resultant orientation image [74]. Either approach can of course be used in conjunction with the filter.

Other researchers have worked with LRO values determined as one of four [79] or eight [78] possible orientations. The algorithm, which determines $L R O$ as one of 16 orientations $\theta_{i}=i \Pi / 16$, $i=0,1,2, \ldots, 15$, i.e. to a precision of $+\Pi / 32$, follows.

\subsubsection{Algorithm for estimating LRO at a point}

A window of size 32 by 32 pixels is centered at the point where the LRO is to be found. This window is rotated to 16 different orientations, $\theta_{i}=i \Pi / 16$, for $i=0,1,2 \ldots 15$. At each orientation a projection along the $y$-axis of the window is formed:

$$
\mathrm{P}_{\mathrm{i}}(\mathrm{x})=\frac{1}{32} \sum_{\mathrm{y}=0}^{31} \mathrm{~W}_{\mathrm{i}}(\mathrm{x}, \mathrm{y}) \quad \mathrm{x}=0,1,2 \ldots 31
$$

where $W_{i}(x, y)$ is the data inside the window at angle $\theta_{i}$.

When the window is aligned with its $x$-axis perpendicular to the ridges, one expects maximum variation of the projection, since ridges are crossed as $\mathrm{x}$ varies. Alignment of the $\mathrm{x}$ - axis along the ridges should lead to minimum variation. This is illustrated in Fig 5-6. 
A second order Butterworth band pass filter removes the noise from the projections. The total variation $\mathrm{V}_{\mathrm{i}}$ of each filtered projection is evaluated as:

$$
V_{i}=\sum_{x=0}^{31}\left|f_{p_{i}}(x+1)-f_{p_{i}}(x)\right|
$$

where

$$
f_{p_{i}}=F F T\left(\mathrm{P}_{\mathrm{i}}(x)\right)
$$

The LRO estimate is given by $i_{\max } \Pi / / 6$, where $V_{i_{\max }}$ is the maximum of the 16 variations. This algorithm produces the correct value except in the noisiest regions. A simple model has been developed describing the behavior of LRO, and incorrect estimates can be dealt with by reference to this model.

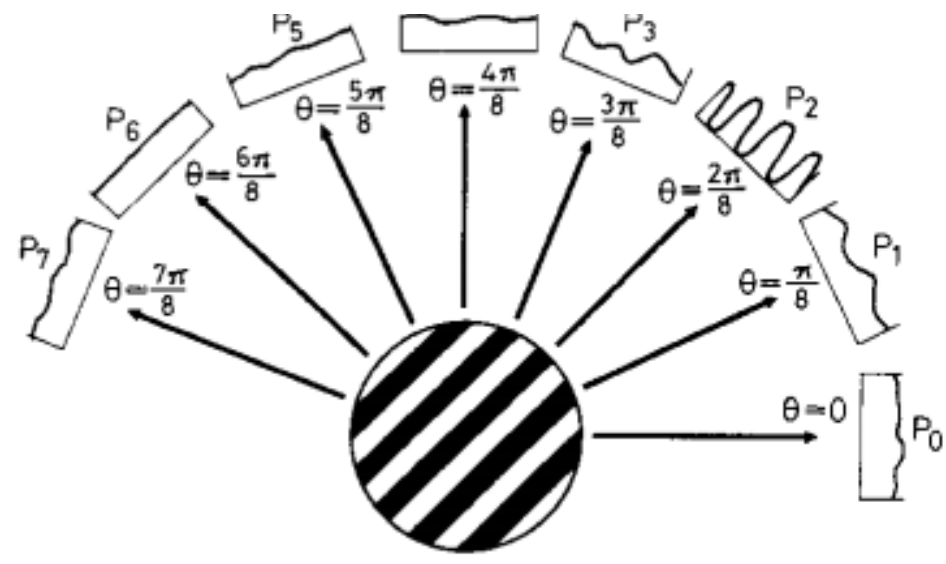

Fig 5-6 Projections of a window of fingerprint image data. The projections which exhibit the greatest variation correspond to the orientation of the ridges within the window (here). Eight projection are shown here [2]. 


\section{$\underline{5.3 \text { Gabor Filter }}$}

In this section the Gabor filter is reviewed. The Gabor filter was originally introduced by Dennis Gabor. The one-dimensional Gabor filter [65] is defined as the multiplication of a cosine/sine (even/odd) wave with Gaussian windows (see Fig 5-7), as follows,

equation 1:

$$
g_{e}(x)=\frac{1}{\sqrt{2 \Pi} \sigma}\left(\exp \left[\frac{-x^{2}}{2 \sigma^{2}}\right]\right) \cdot \cos \left(2 \pi x \omega_{0}\right)
$$

eqation 2:

$$
g_{o}(x)=\frac{1}{\sqrt{2 \Pi} \sigma}\left(\exp \left[\frac{-x^{2}}{2 \sigma^{2}}\right]\right) \cdot \sin \left(2 \pi x \omega_{0}\right)
$$

where $\omega_{0}$ defines the centre frequency (i.e., the frequency in which the filter yields the greatest response) and $\sigma$ is the spread of the Gaussian window. The power spectrum of the Gabor filter is given by the sum of two Gaussians centred at $\pm \omega_{0}$

$$
|| G(\omega) \|=\exp \left[-2 \pi^{2} \sigma^{2}\left(\omega-\omega_{0}\right)^{2}\right]+\exp \left[-2 \pi^{2} \sigma^{2}\left(\omega+\omega_{0}\right)^{2}\right]
$$

This can be reasoned as follows. The power spectrum of a sine wave has two impulses located at $\pm \omega_{0}$ and the power spectrum of Gaussian is a (non-normalized) Gaussian. Multiplication in the temporal (spatial) domain is equivalent to convolution in the frequency domain [82].

The uncertainty principle states that the product of the spread (i.e., uncertainty) of a signal in the time and frequency domains must exceed or equal a fixed constant,

$$
\Delta \mathrm{t} \Delta \mathrm{f} \geq \mathrm{c}
$$




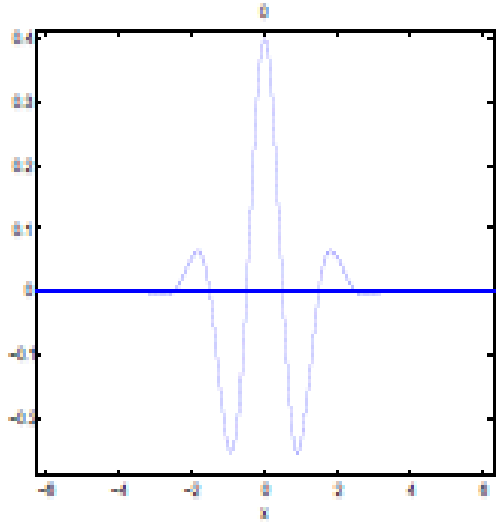

(a) Gabor cosine (even)

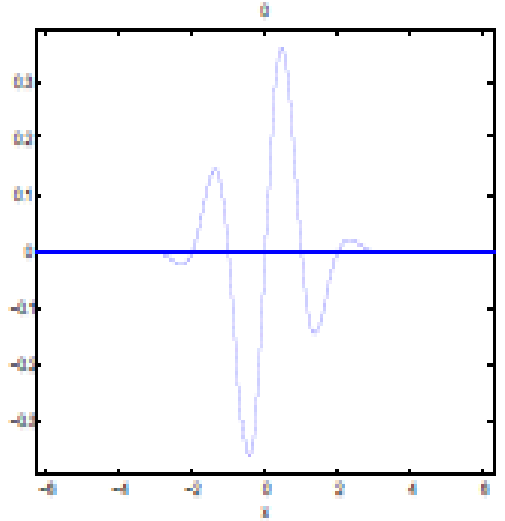

(b) Gabor sine (odd)

Fig 5-7 One dimensional Gabor filter [65]

where $c$ is a constant, $t$ and $f$ represent the measure of the spread of the signal in the time and frequency domains, respectively (see Fig 5-8). The implication of this principle is that the accuracy with which one can measure a signal in one domain limits the attainable accuracy of the measurement in the other domain. Gabor demonstrated that the complex Gabor filter given by,

equation 3:

$$
\mathrm{g}(x)=\mathrm{g}_{\mathrm{e}}(x)+\operatorname{jg}_{\mathrm{o}}(x)
$$

substituting equation 1 and equation 2 in equation 3 :

$$
g(x)=\frac{1}{\sqrt{2 \Pi} \sigma}\left(\exp \left[\frac{-x^{2}}{2 \sigma^{2}}\right]\right) \cdot \cos \left(2 \pi x \omega_{0}\right)+j \frac{1}{\sqrt{2 \Pi} \sigma}\left(\exp \left[\frac{-x^{2}}{2 \sigma^{2}}\right]\right) \cdot \sin \left(2 \pi x \omega_{0}\right)
$$

Since $\exp [j \theta]=\cos \theta+j \sin \theta$

$$
g(x)=\frac{1}{\sqrt{2 \Pi \sigma}}\left(\exp \left[\frac{-x^{2}}{2 \sigma^{2}}\right]\right) \cdot \operatorname{expj}\left[\left(2 \pi x \omega_{0}\right)\right]
$$


attains the optimal (lower bound) compromise between the localization in the time and frequency domains; notice that the Gaussian function is an instance of a Gabor filter with center frequency $\omega_{0}=0$. Note that the constituent real-valued Gabor elementary functions (i.e., the even and odd parts taken separately) do not as widely believed minimize the joint uncertainty. Furthermore, the selection of a different localization measure may result in a different class of "optimal" function altogether casting doubt on the primacy of the Gabor function often cited in the literature.

Daugman extended the Gabor filter to two- dimensions (see Fig 5-9), as follows,

$$
\begin{aligned}
& \mathrm{g}_{\mathrm{e}}(x, \mathrm{y})=\frac{1}{2 \pi \sigma_{x} \sigma_{\mathrm{y}}} \exp \left[-\frac{1}{2}\left(\frac{x^{2}}{\sigma_{x}}+\frac{\mathrm{y}^{2}}{\sigma_{\mathrm{y}}}\right)\right] \cdot\left[\cos \left(2 \pi \omega_{x 0} x+2 \pi \omega_{\mathrm{y} 0} \mathrm{y}\right)\right] \\
& \mathrm{g}_{\mathrm{o}}(x, \mathrm{y})=\frac{1}{2 \pi \sigma_{x} \sigma_{\mathrm{y}}} \exp \left[-\frac{1}{2}\left(\frac{x^{2}}{\sigma_{x}}+\frac{\mathrm{y}^{2}}{\sigma_{\mathrm{y}}}\right)\right] \cdot\left[\sin \left(2 \pi \omega_{x 0} x+2 \pi \omega_{\mathrm{y} 0} \mathrm{y}\right)\right]
\end{aligned}
$$

where $\left(\omega_{x 0}, \omega_{\mathrm{y} 0}\right)$ defines the center frequency and $\left(\sigma_{\square}, \sigma_{\mathrm{y}}\right)$ the (potentially asymmetric) spread of the Gaussian window.

For the purpose of extracting optical flow, Heeger [87] utilized the three-dimensional (space-time) Gabor filter,

$$
\begin{aligned}
& g_{e}(x, y, t)=\frac{1}{\left[(2 \pi)^{3 / 2}\right] \sigma_{x} \sigma_{y} \sigma_{t}} \exp \left[-\frac{1}{2}\left(\frac{x^{2}}{\sigma_{x}}+\frac{y^{2}}{\sigma_{y}}+\frac{t^{2}}{\sigma_{t}}\right) \cos \left(2 \pi \omega_{x 0} x+2 \pi \omega_{y 0} y+2 \pi \omega_{t 0} t\right)\right] \\
& g_{e}(x, y, t)=\frac{1}{\left[(2 \pi)^{3 / 2}\right] \sigma_{x} \sigma_{y} \sigma_{t}} \exp \left[-\frac{1}{2}\left(\frac{x^{2}}{\sigma_{x}}+\frac{y^{2}}{\sigma_{y}}+\frac{t^{2}}{\sigma_{t}}\right) \sin \left(2 \pi \omega_{x 0} x+2 \pi \omega_{y 0} y+2 \pi \omega_{t 0} t\right)\right]
\end{aligned}
$$




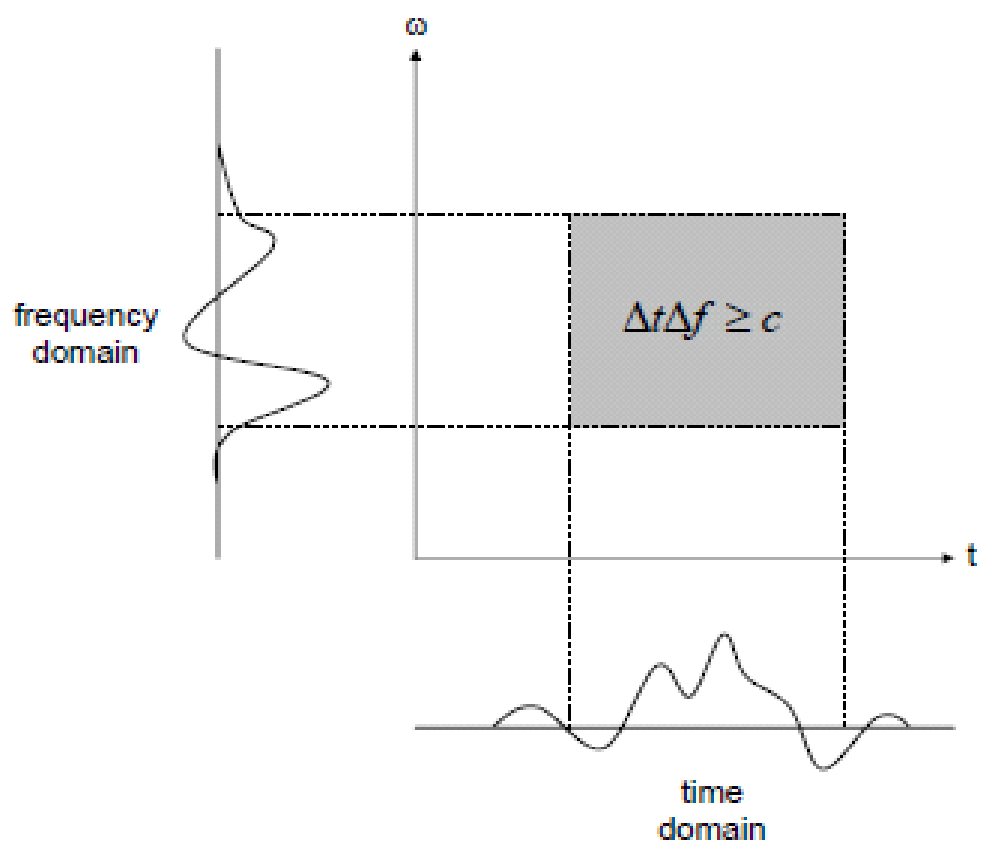

Fig 5-8 Joint localization of a signal in time and frequency domain [65]

where $\left(\omega_{x 0}, \omega_{y 0}, \omega_{t 0}\right)$ defines the center frequency and $\left(\sigma_{x}, \sigma_{y}, \sigma_{t}\right)$ defines the (potentially asymmetric) spread of the Gaussian window.

Heeger demonstrated that the three-dimensional (similarly for the two-dimensional case) Gabor filter can be built from one-dimensional separable components. Considering the twodimensional Gabor filter let $\mathrm{k}$ be the size of the two-dimensional convolution kernel and $\mathrm{n}$ be the size of an image (in pixels). The complexity of the non-separable convolution of the Gabor filter is reduced from $O(k, n)^{2}$ to $O\left(k n^{2}\right)$.

An application of Gabor filters is in local time-frequency analysis of signals, specifically, a fixed windowed Fourier transform, referred to as the Gabor transform. A difficulty with the Gabor transform is that it is linearly independent but highly non-orthogonal and as such cannot be easily inverted. As a result of non-orthogonality, the functions, $r(n)$, used for reconstructing the discrete signal, $f(n)$, are highly distinct from the Gabor functions used to recover the 
coefficients of the representation (i.e., the analysis step), where the coefficients indicate how much of its corresponding reconstruction filter, $r(n)$, is to be added, formally,

$f(n)=\sum_{i} c_{i} r_{i}(n) \quad$ reconstruction step

where

$c_{i}=\sum_{i} f(n) g_{i}(n) \quad$ analysis step

If the Gabor transform is indeed orthogonal (such as the Fourier transform), then

$$
g_{i}(n)=r_{i}(n)
$$

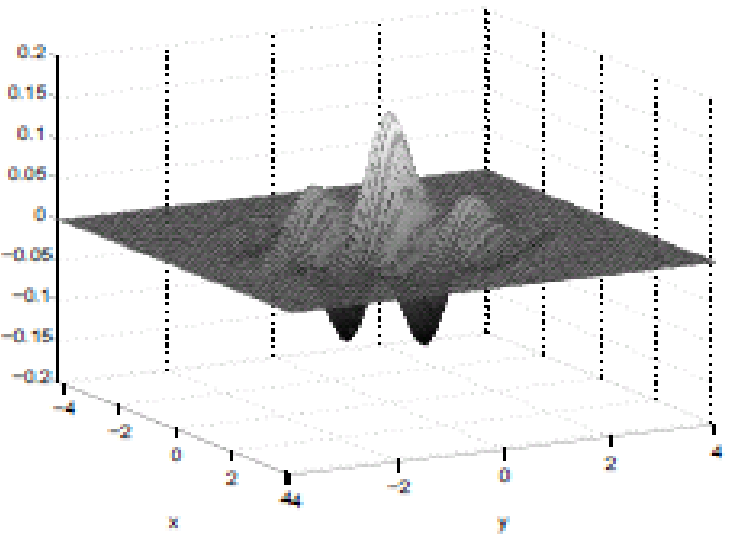

(a) Cabor cusine (even)

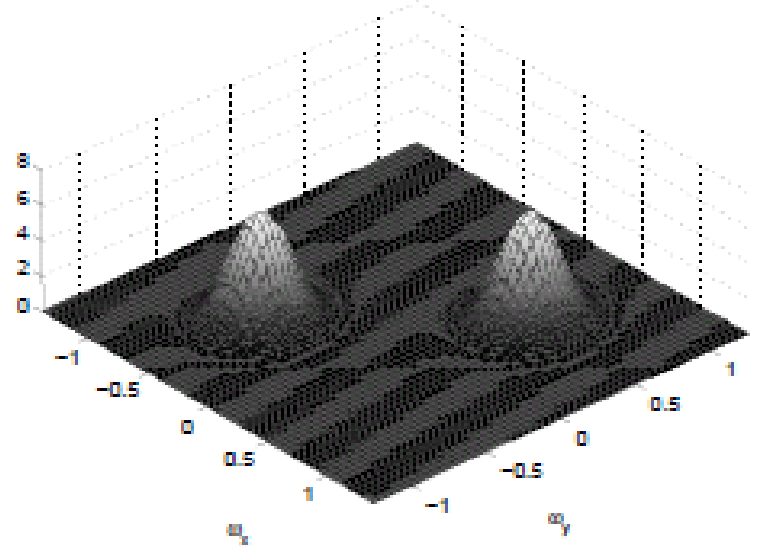

(b) Gabar powor spoctrum

Fig 5-9 Two dimensional Gabor filters [65] 
Gabor filters optimally capture both local orientation and frequency information from a fingerprint image. By tuning a Gabor filter to specific frequency and direction, the local frequency and orientation information can be obtained .

An even symmetric Gabor filter has the following general form in the spatial domain:

$$
\begin{array}{lr}
g(x, y ; f, \theta)=\exp \left\{\frac{-1}{2}\left[\frac{x^{\prime^{2}}}{\mathfrak{d}^{2}}+\frac{y^{\prime}}{\mathfrak{d}^{2}}{\frac{y^{\prime}}{}}^{2}\right]\right\} \cos \left(2 \Pi f x^{\prime}\right) & \text { equation } 1 \\
x^{\prime}=x \sin \theta+y \cos \theta & \text { equation 2 } \\
y^{\prime}=x \cos \theta-y \sin \theta & \text { equation 3 }
\end{array}
$$

where $f$ is the frequency of the sinusoidal plane wave along the direction $\theta$ from the $x$ axis and $D_{x}$ and $D_{y}$ are the space constants of the Gaussian envelop along the $x$ and $y$ axes respectively. The filtering is performed in the spatial domain with a mask size of $32 \times 32$. The frequency $f$ is the average ridge frequency $(1 / K)$, where $K$ is the average inter ridge distance. The average inter ridge distance is approximately 10 pixels in a 500 dpi fingerprint image. Hence, $f=1 / 10$. Sixteen different orientations are examined.

In this thesis the Gabor filter is applied to the original image after it is normalized and orientation is performed. 


\subsection{Results}

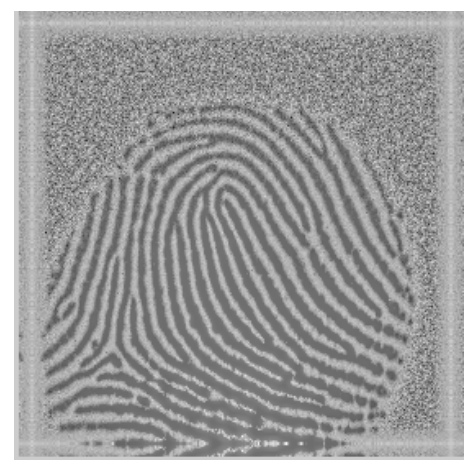

(a)

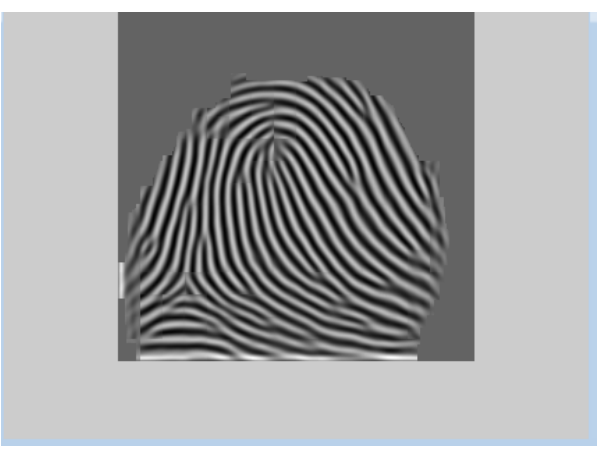

(b)

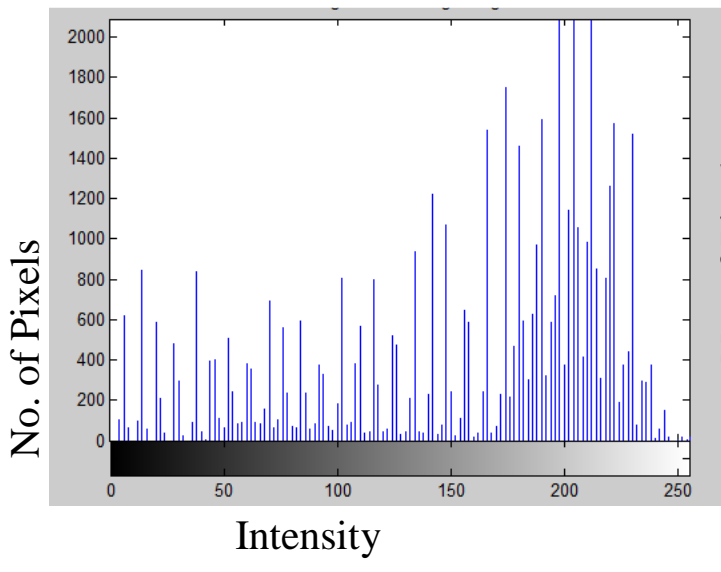

(c)

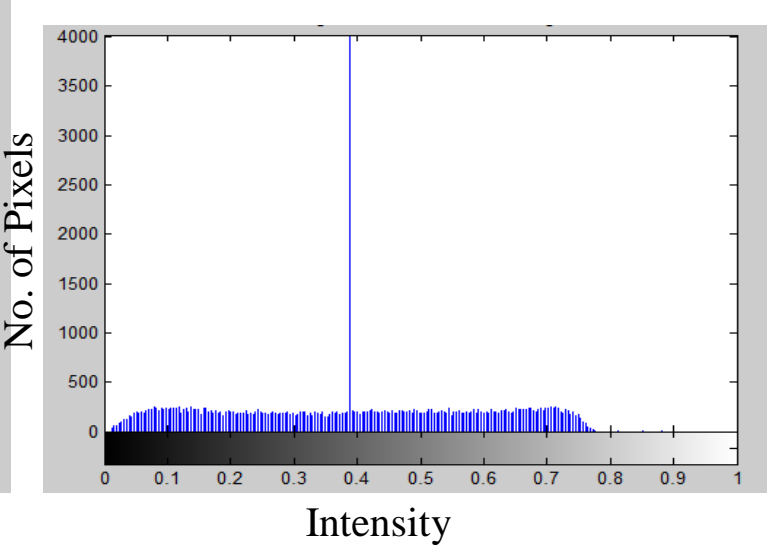

(d)

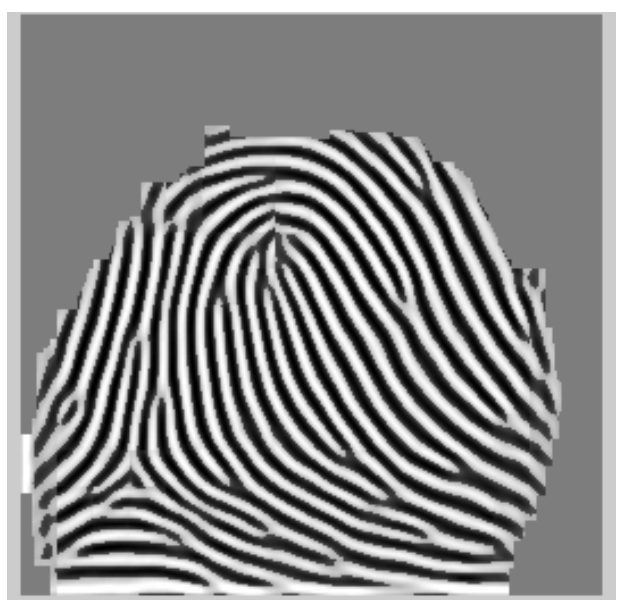

(e)

Fig 5-10 Image 1(a) Original image (b) Gabor filtered image (c) Histogram of the original image (d) Histogram of the Gabor filtered image (e) Histogram equalized of the Gabor filtered image 


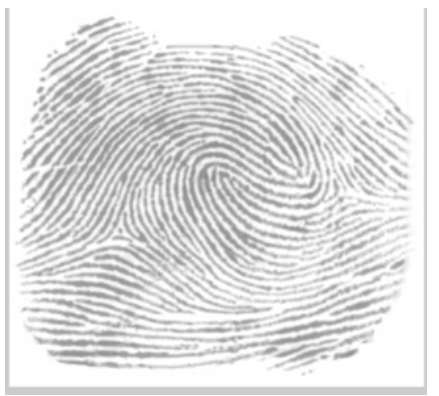

(a)

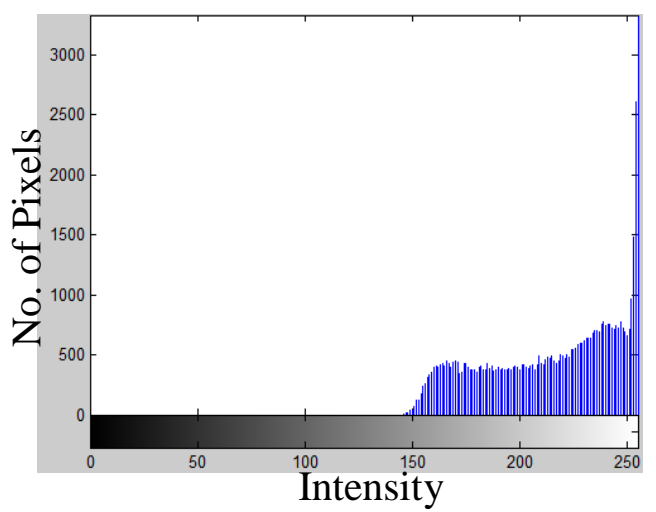

(c)

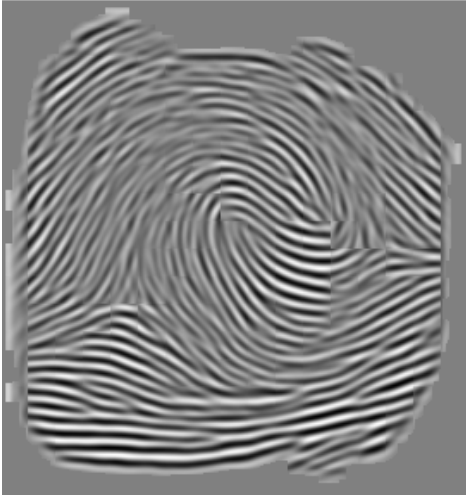

(b)

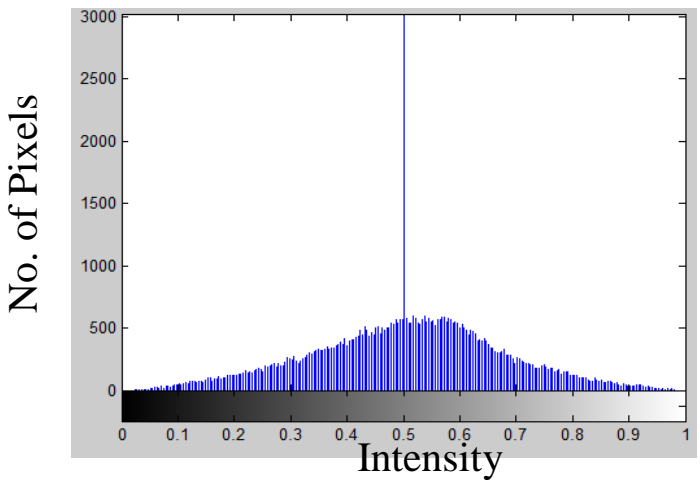

(d)

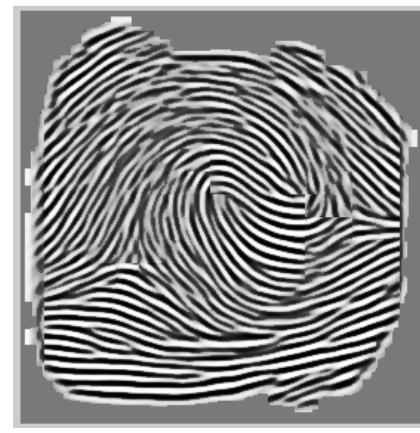

(e)

Fig 5-11 Image 2(a) Original image (b) Gabor filtered image (c) Histogram of the original image (d) Histogram of the Gabor filtered image (e) Histogram equalized of the Gabor filtered image 


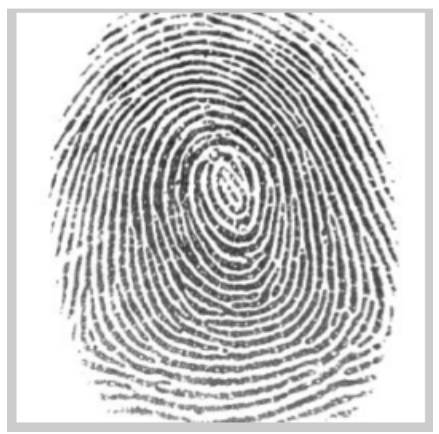

(a)

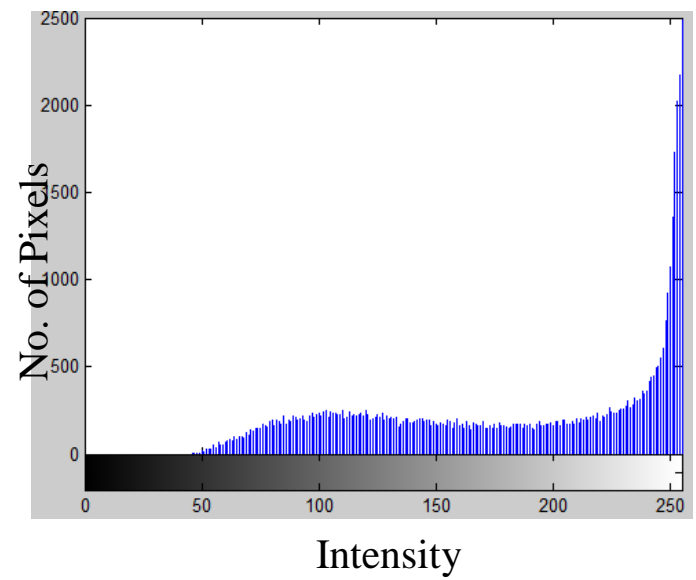

(c)

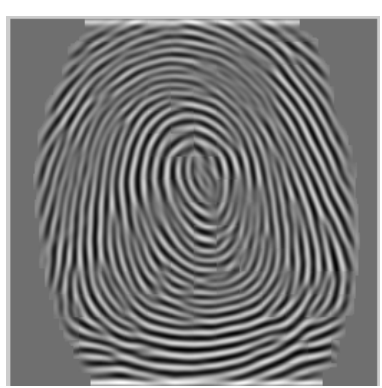

(b)

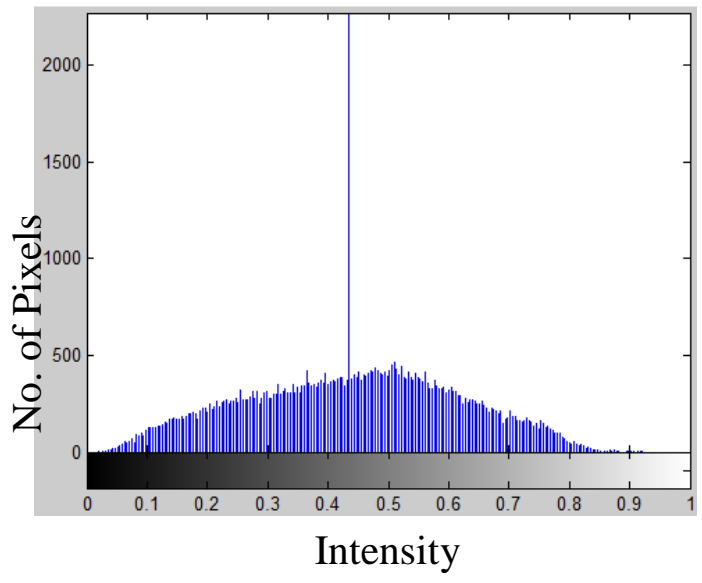

(d)

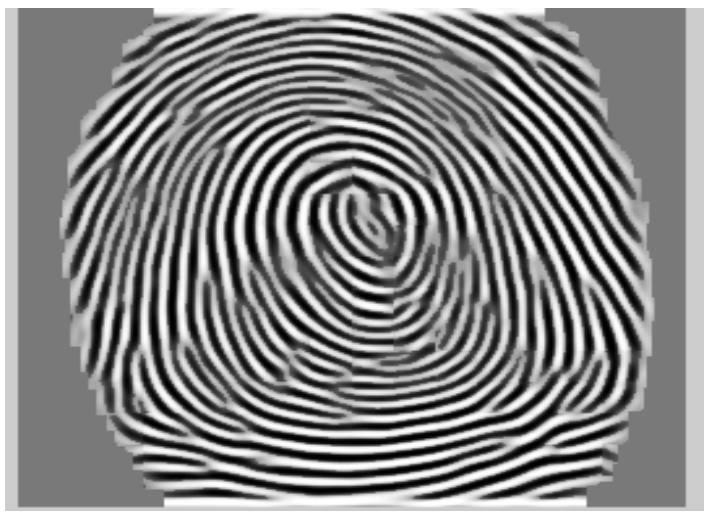

(e)

Fig 5-12 Image 3(a) Original image (b) Gabor filtered image (c) Histogram of the original image (d) Histogram of the Gabor filtered image (e) Histogram equalized of the Gabor filtered image 


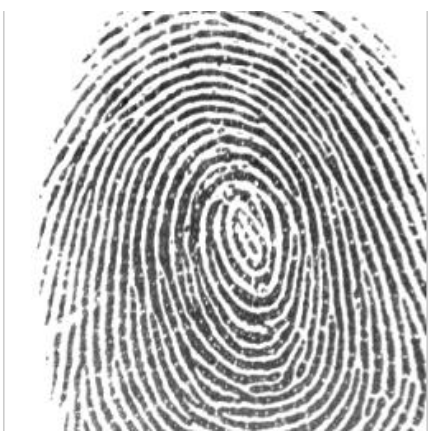

(a)

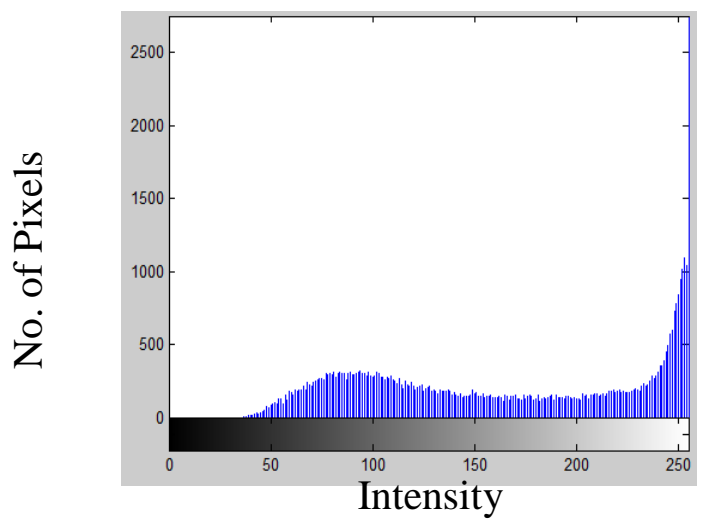

(c)

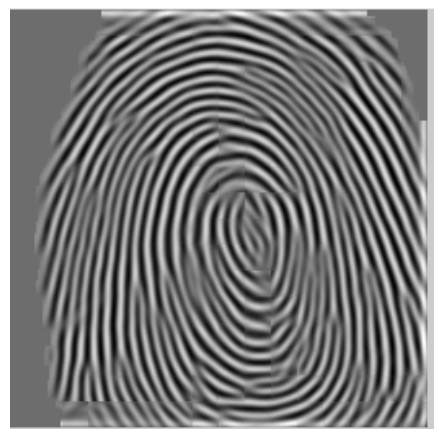

(b)

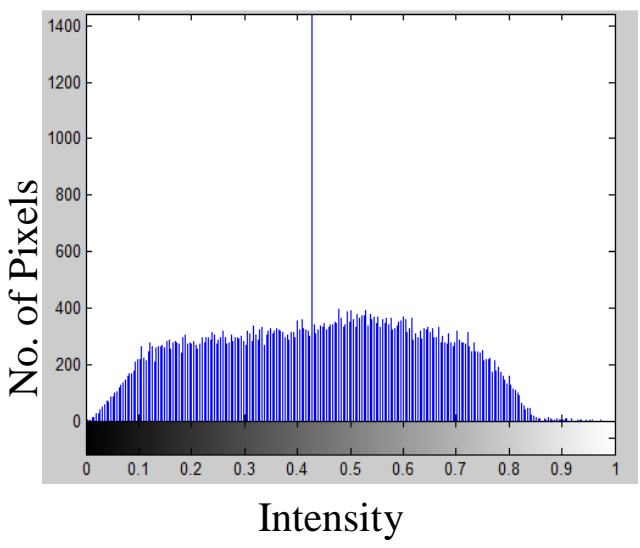

(d)

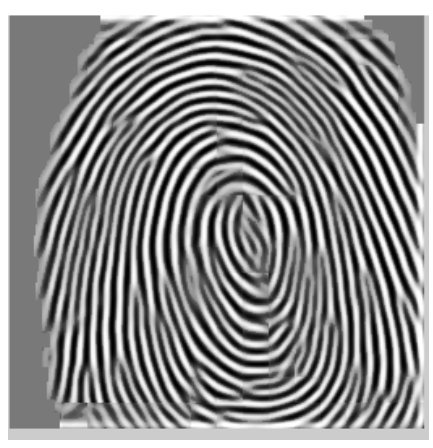

(e)

Fig 5-13 Image 4(a) Original image (b) Gabor filtered image (c) Histogram of the original image (d) Histogram of the Gabor filtered image (e) Histogram equalized of the Gabor filtered image 


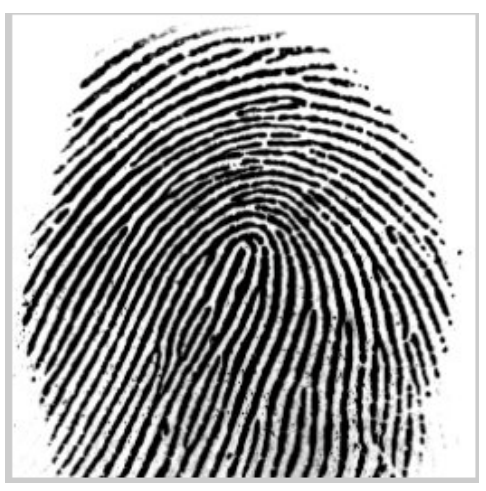

(a)

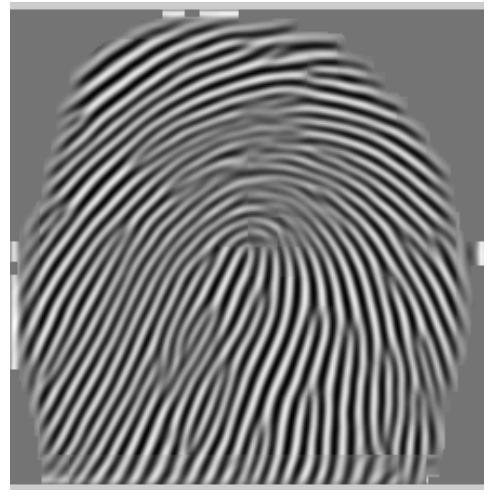

(b)

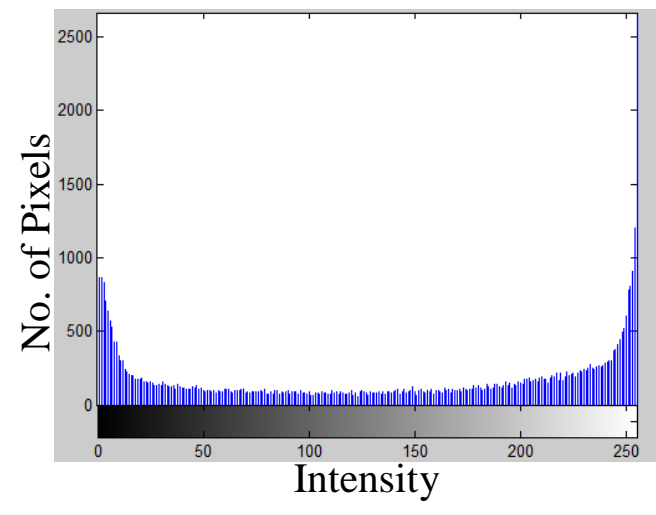

(c)

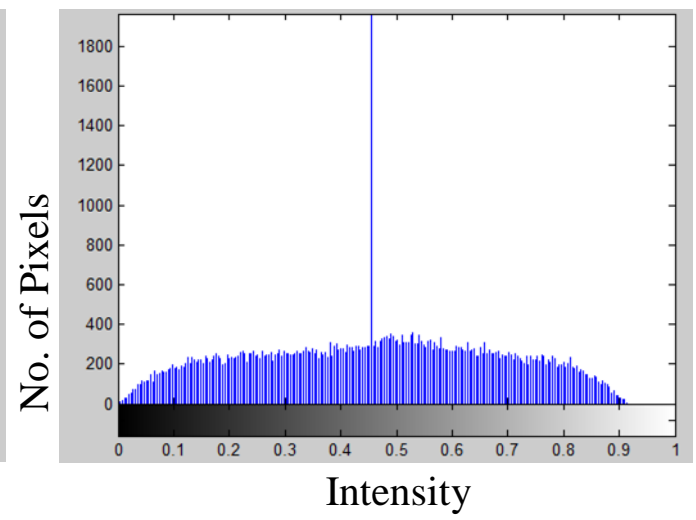

(d)

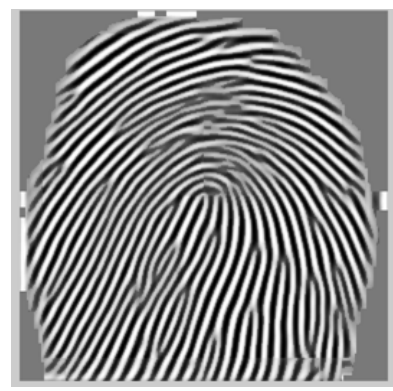

(e)

Fig 5-14 Image 5(a) Original image (b) Gabor filtered image (c) Histogram of the original image (d) Histogram of the Gabor filtered image (e) Histogram equalized of the Gabor filtered image 


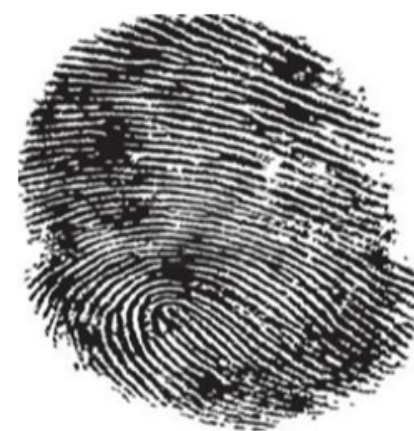

(a)

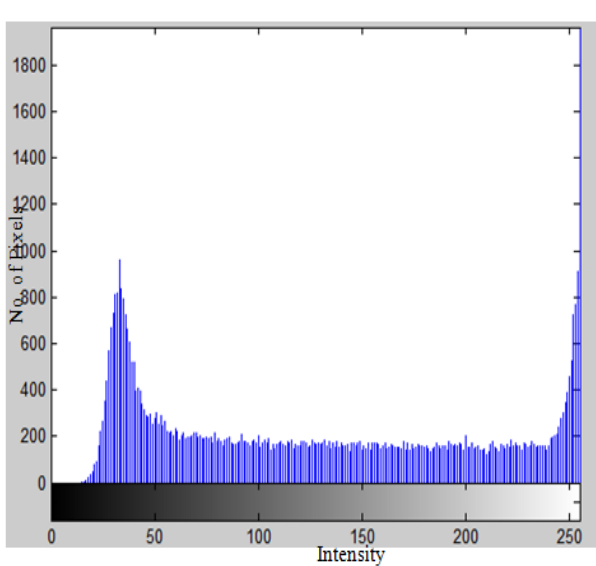

(c)

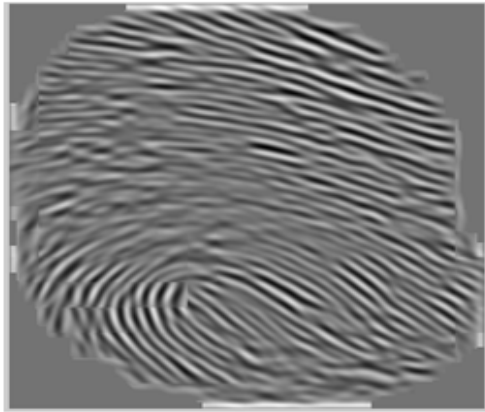

(b)

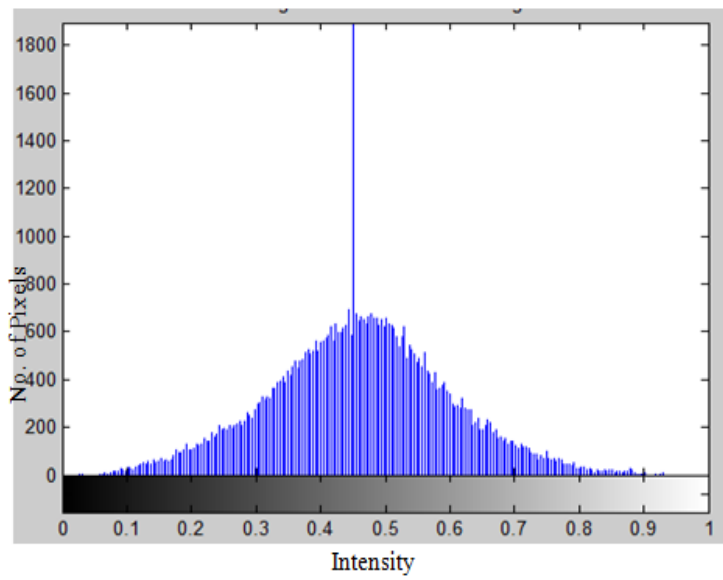

(d)

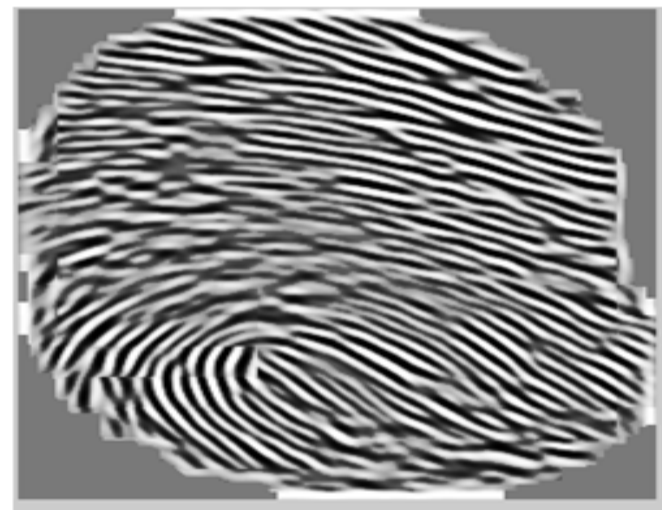

(e)

Fig 5-15 Image 6(a) Original image (b) Gabor filtered image (c) Histogram of the original image (d) Histogram of the Gabor filtered image (e) Histogram equalized of the Gabor filtered image 


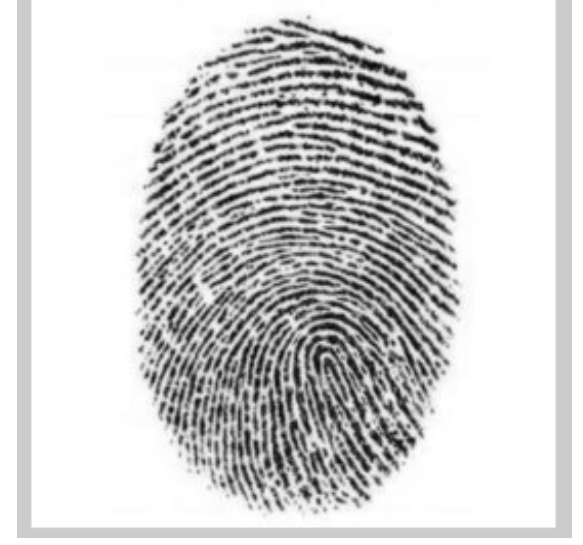

(a)

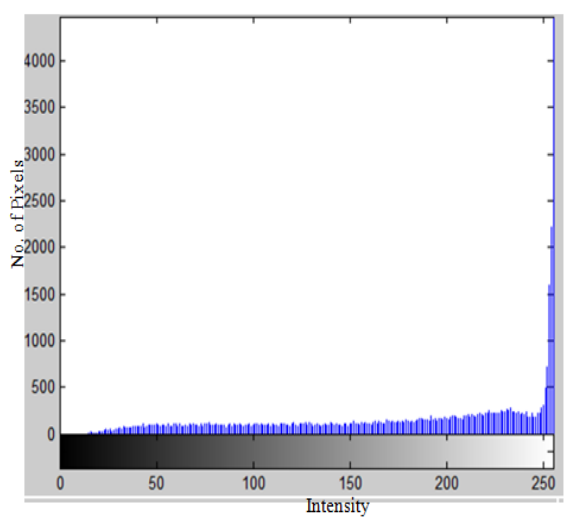

(c)

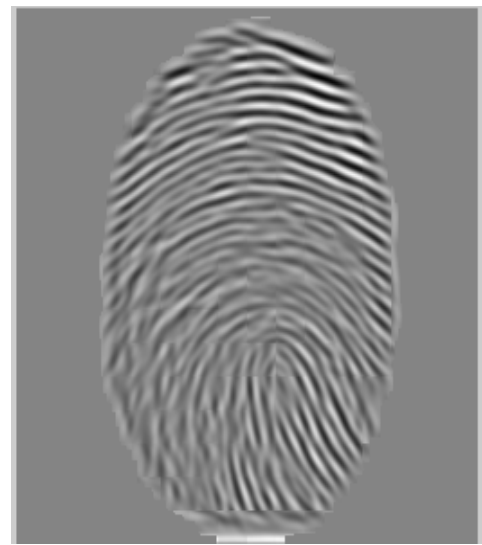

(b)

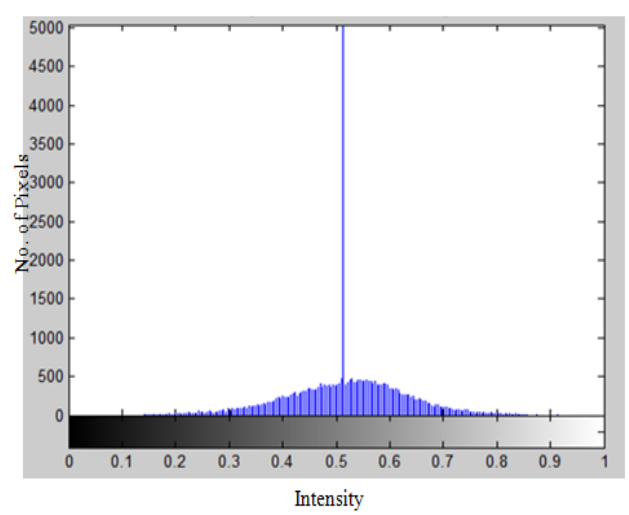

(d)

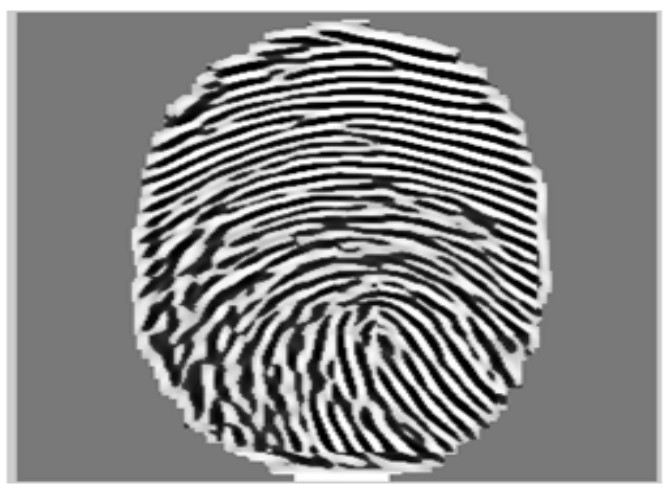

(e)

Fig 5-16 Image 7(a) Original image (b) Gabor filtered image (c) Histogram of the original image (d) Histogram of the Gabor filtered image (e) Histogram equalized of the Gabor filtered image 


\section{$\underline{5.5 \text { Histogram }}$}

Contrast of an image is determined by its dynamic range, which is defined as a ratio between the brightest and the darkest pixel intensities. Contrast enhancement techniques had various application areas for enhancing visual quality of low contrast images. Original and final image histograms are shown in Fig 5-120c and Fig 5-120d respectively.

\subsection{Histogram equalization}

Histogram equalization is a general process used to enhance the contrast of images by transforming its intensity values. The spread of the histogram over the entire intensity is increasing the contrast and the average intensity level in the histogram of the equalized image is higher (lighter) than the original. Histogram equalization has been applied to the enhanced image as shown in Fig 5-120e.

\section{$\underline{5.7 \text { Verification }}$}

For comparison, the images are shown: enhanced with the STFT method [24]

(MATLABcodeavailablefromhttp://www.cubs.buffalo.edu/code

Table 1: Comparison of number of minutiae for various enhancement approaches

\begin{tabular}{|l|l|}
\hline Fig. & A \\
\hline Without enhancement & 188 \\
\hline STFT & 132 \\
\hline Gabor filter & 118 \\
\hline
\end{tabular}

A - Automatically extracted minutiae 


\section{$\underline{5.8 \text { Conclusions }}$}

In this thesis Gabor filter is used for fingerprint enhancement technique. Because of its frequency selective and orientation selective properties it proves to be useful for fingerprint enhancement. A window of size 32 by 32 pixels is centered at the point where the LRO is to be found. This window is rotated to 16 different orientations. The projections which exhibit the greatest variation corresponds to the orientation of the ridges within the window. The primary advantage of the approach is improved translation and rotation invariance. Future work would involve making the fingerprint enhancement technique more efficient. 
APPENDIX A

GABOR FILTER 
An even symmetric Gabor filter has the following general form in the spatial domain:

$g(x, y ; f, \theta)=\exp \left\{\frac{-1}{2}\left[\frac{x^{\prime 2}}{\mathfrak{d}_{x^{\prime}}^{2}}+\frac{y^{\prime^{2}}}{\mathfrak{d}_{y^{\prime}}^{2}}\right]\right\} \cos \left(2 \Pi f x^{\prime}\right)$

equation A.1

$x^{\prime}=x \sin \theta+y \cos \theta$

equation A.2

$y^{\prime}=x \cos \theta-y \sin \theta$

equation A.3

where $f$ is the frequency of the sinusoidal plane wave along the direction $\theta$ from the $x$ axis and $D_{x}$ and $y$ are the space constants of the Gaussian envelop along the $x$ and $y$ axes respectively. The filtering is performed in the spatial domain with a mask size of $32 \times 32$. The frequency $f$ is the average ridge frequency $(1 / K)$, where $K$ is the average inter ridge distance. The average inter ridge distance is approximately 10 pixels in a 500 dpi fingerprint image. Hence, $f=1 / 10$. Sixteen different orientations are examined. 


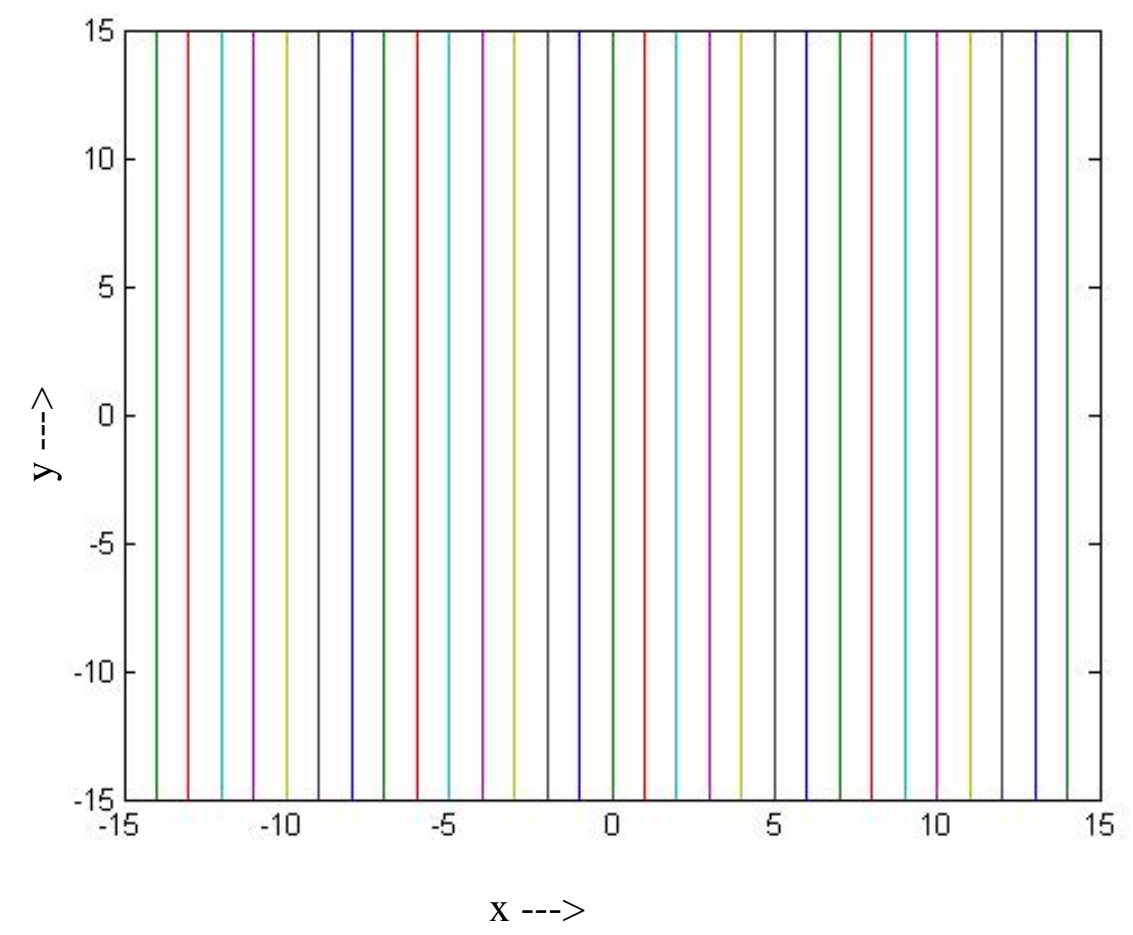

Fig A-1 The range of $x$ and $y$ goes from -15 to 15 to produce the Gabor kernel 
(a) block $-32 \times 32$

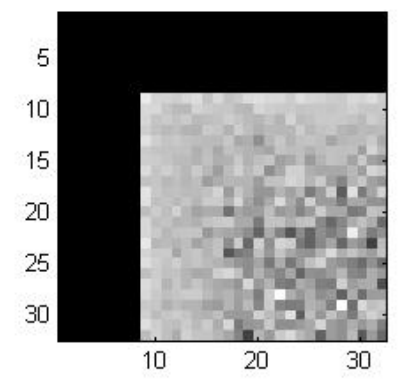

(b)FFT of

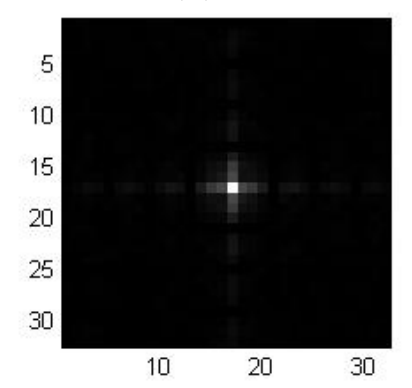

(c) Gabor Kernel - 32x32

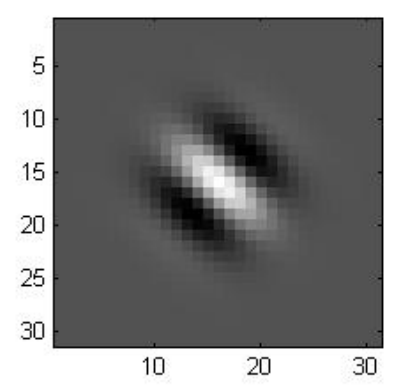

(d)FFT of Gabor

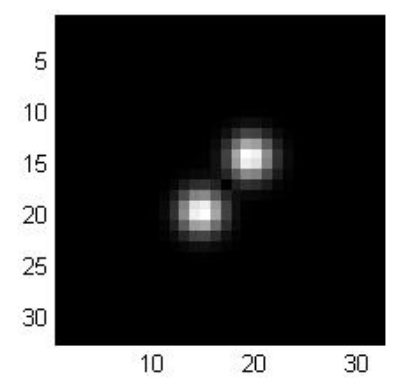

Fig A-2(a) block - 32×32 (b)FFT of block (c) Gabor Kernel - 32×32 (d)FFT of Gabor Kernel

We input different values of $x$ and $y$ from fig A.1 which varies from -15 to +15 and different orientation values and substitute them in equation A.2 and A.3. Then after that we input those values from equation A.2 and A.3 in equation A.1 to obtain Gabor kernels (32x32) (fig.A.2 (c)). Then we convolve the Fourier image and Fourier filter bank (fig A.3).

Let $\mathrm{I}(\mathrm{x}, \mathrm{y})$ be the image Let $g(x, y, f$, theta) be the Gabor filter Let $F($.$) be the Fourier transform and Finv(.) be the inverse$ Fourier transform. Let * be the convolution and . be pointwise multiplication.

$I(x, y)^{*} g(x, y, f$, theta $)=F i n v(F(I(i, x)) \cdot F(g(x, y, f$, theta $)))=$ Gabor filtered image. 


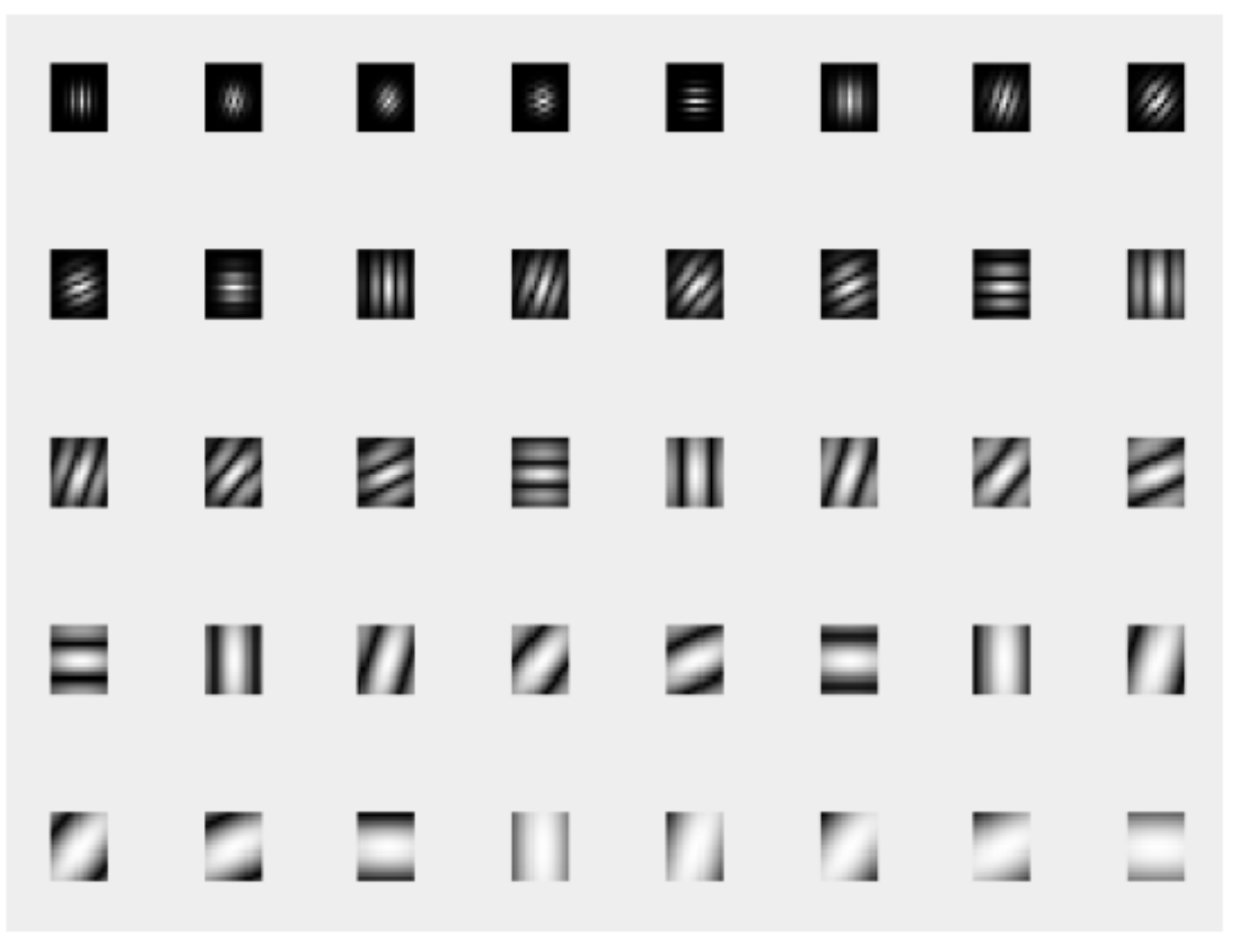

Fig A-3 Gabor filters of size $16 \times 16$ by 8 orientations and 5 Resolutions (real part). 


\section{REFERENCES}

[1] A.M.Raičević and B.M. Popović, "An effective and robust enhancement by adaptive filtering domain", SER.:ELEC.ENERG. vol.22, no. 1, pp.91-104, April 2009.

[2] B.G. Sherlock, D.M. Monro, and K. Millard, "Fingerprint enhancement by directional Fourier filtering”, IEE Proc. Vision Image Signal Process., vol.141, no. 2, pp. 87-94, April 1994.

[3] L. Hong, Y.Wan, and A.K. Jain, "Fingerprint image enhancement: Algorithm and performance evolution", IEEE Trans. PAMI, vol. 20, no. 8, pp. 777-789, Aug. 1998.

[4] J.Yang, L. Lin, T. Jiang, and Y.Fan, "A modified Gabor filter design method for fingerprint image enhancement”, Pattern Recognition Letters, vol. 24, no. 12, pp. 1805-1817, Jan. 2003.

[5] A.K. Jain and F. Farrokhnia, "Unsupervised texture segmentation using Gabor filters", Pattern Recognition, vol. 24, no. 12, pp. 1167-1186, May 1991.

[6] K. Karu and A.K. Jain, "Fingerprint classification", Pattern Recognition, vol.29, no. 3, pp. 389-404, Mar. 1996.

[7] Fingerprint database, "http://www nist.gov/it/iad/ig/sd27a.cfm"

[8] A.L Bovik, Handbook of Image and Video Processing. Elsevier, 2005.

[9] K.R.Rao, D.N.Kim and J.J.Hwang, "Fast Fourier transform: Algorithms and applications", Heidelberg, Germany: Springer 2010.

[10] C.M. Brislawn, et al, "The FBI compression standard for digitized fingerprint images", Proceedings of SPIE, vol. 2847, pp. 344-355, Aug. 1996.

[11]A. K. Jain et al, "An identity authentication system using fingerprints", Proc. IEEE, vol. 85, no. 9, pp. 1365-1388, Sep. 1997. 
[12] American National Standards Institute, "American national standard for information systems-data format for the interchange of fingerprint information", 1993. Doc\# ANSI/NIST-CSL 1-1993.

[13]H.C. Lee and R.E. Gaensslen, "Advances in fingerprint technology", Elsevier, New York, 2nd edition, 2001.

[14] S. Pankanti, S. Prabhakar, and A. K. Jain, "On the individuality of finger print", IEEE Transaction on PAMI, vol. 24, no. 8, pp. 1010-1025, Aug. 2001.

[15]D. A. Stoney and J. I. Thornton, "A critical analysis of quantitative fingerprint individuality models", Journal of Forensic Sciences, vol. 31, no. 4, pp. 1187-1216, Oct. 1986.

[16]D. Maio, et al, "FVC2002: Second fingerprint verification competition", International Conference on Pattern Recognition (16th), vol. 3, pp. 811-814, Dec 2002.

[17] American National Standards Institute Common biometric exchange file format (http://www.itl.nist.gov/div895/isis/bc/cbeff/).

[18] S. Chikkerur, C. Wu, and V. Govindaraju, "A systematic approach for feature extraction in fingerprint images", International Conference on Biometric Authentication, vol. 3072, pp. 123, 2004.

[19] L. Hong, Y. Wang, and A. K. Jain, "Fingerprint image enhancement: Algorithm and performance evaluation", IEEE Transactions on PAMI, vol. 21, no. 4, pp.777-789, Aug. 1998.

[20]S.Greenberg, et al, "Fingerprint image enhancement using filtering techniques", International Conference on Pattern Recognition, vol. 3, pp. 326-329, Sep. 2000.

[21]C. Wu, Z. Shi, and V. Govindaraju, "Fingerprint image enhancement using directional median filters", Proceeding of SPIE, vol. 5404, pp. 66-75, Feb. 2004.

[22] Finger print Scanners, "http://360biometrics.com/faq/fingerprint_scanners.php\#5" 
[23]A. J. Willis and L. Myers, "A cost-effective fingerprint recognition system for use with low-quality prints and damaged fingertips", Pattern Recognition, vol. 34, no. 2, pp.255-270, Feb. 2001.

[24]S. S. Chikkerur, "Online fingerprint verification", Master's thesis, The State University of New York at Buffalo, 2005.

[25] C.-T. Hsieh, E. Lai, and Y.C. Wang, "An effective algorithm for fingerprint image enhancement based on wavelet transform", Pattern Recognition, vol. 36, no. 2, pp.303312, Feb. 2003.

[26]X. Jiang and W.Y. Yau, "Fingerprint minutiae matching based on the local and global structures", Int. Conf. on Pattern Recognition (15th), volume 2, pp. 1042-1045, Aug. 2000.

[27] D. Lee, K.Choi, and J. Kim, "A robust fingerprint matching algorithm using local alignment" ,16th International Conference on Pattern Recognition, vol. 3, pp. 803-806, Dec. 2002.

[28] S. Prabhakar, A.K. Jain, and S. Pankanti, "Learning fingerprint minutiae location and type", Pattern Recognition, vol. 36, no. 8, pp. 1847-1857, Feb. 2003.

[29] D. P. Mital and E. K. Teoh, "An automated matching technique for fingerprint identification", First Int. Conf. on Knowledge-Based Intelligent Electronic Systems, vol.1, pp. 142-147, Aug. 1997.

[30] M.D. Garris, et al, "User's guide to NIST fingerprint image software (NFIS)", Technical Report NISTIR 6813, National Institute of Standards and Technology, 2002.

[31] C. I. Watson, et al, "User's guide to NIST fingerprint image software 2 (NFIS2)", Technical report, National Institute of Standards and Technology, 2002.

[32] M. Sezgin and B. Sankur, "Survey over image thresholding techniques and quantitative performance evaluation", Journal of Electronic Imaging, vol.13, no. 1, pp. 146- 165, Jan. 2004.

[33] O.D. Trier and T. Taxt, "Evaluation of binarization methods for document images", IEEE Transactions on PAMI, vol. 17, no. 3, pp. 312-315, Mar 1995. 
[34] L. Dong and G. Yu, "An optimization-based approach to image binarization”, The Fourth International Conference on Computer and Information Technology, pp. 165-170, Sept. 2004.

[35]N. Otsu, "A threshold selection method from gray level histograms", IEEE Transactions on Systems, Man and Cybernetics, vol. 9, no. 1, pp. 62-66, Jan. 1979.

[36]M. Stock and C.W. Swonger, "Development and evaluation of a reader of fingerprint minutiae", Technical Report XM-2478-X1:13-17, Cornell Aeronautical Laboratory, 1969.

[37] N.K. Ratha, S.Y. Chen, and A.K. Jain, "Adaptive flow orientation-based feature extraction in fingerprint images", Pattern Recognition, vol. 28, no. 11, pp. 1657-1672, Nov. 1995.

[38] L. Coetzee and E. C. Botha, "Fingerprint recognition in low quality images", Pattern Recognition, vol. 26, no. 10, pp. 1441-1460, Oct. 1993.

[39]B. Moayer and K. Fu, "A tree system approach for fingerprint pattern recognition", IEEE Transactions on PAMI, vol. 8, no. 3, pp.376-388, May 1986.

[40]M.R. Verma, A.K. Majumdar, and B.Chatterjee, "Edge detection in fingerprints", Pattern Recognition, vol. 20, no.7, pp.513-523, Feb. 1987.

[41]R. Snelick, et al, "Multimodal biometrics: issues in design and testing" ICMI '03: Proceedings of the 5th international conference on Multimodal interfaces, pp. 68-72, NY, USA, Nov. 2003.

[42] C. Domeniconi, S. Tari and P. Liang, "Direct gray scale ridge reconstruction in fingerprint images", IEEE International Conference Acoustics Speech and Signal Processing, vol. 5, pp. 2941-2944, May 1998.

[43]A.S. Abutaleb and M. Kamel, "A genetic algorithm for the estimation of ridges in fingerprints", IEEE Transactions on Image Processing, vol. 8, no. 8, pp. 1134-1138, Aug. 1999.

[44]M.Tico and P. Kuosmanen, "A topographic method for fingerprint segmentation", International Conference on Image Processing, vol. 1, pp. 36-40, Oct. 1999. 
[45]X. Luo and J. Tian, "Knowledge based fingerprint image enhancement", Proceedings of International Conference on Pattern Recognition (15th), vol. 4, pp. 783-786, Sep. 2000.

[46] I. Moccagatta, M.Z. Coban and H.H. Chen, "Wavelet-based image coding: Comparison of MPEG-4 and JPEG-2000", Conference record of the thirty-third asilomar conference on Signals, Systems, and Computers, vol. 2, pp. 1178-1182, Oct. 1999

[47] A.M. Bazen, et al, "A correlation-based fingerprint verification system", Proceedings 11th Annual Workshop Circuits Systems and Signal Processing, pp. 205-213, Nov. 2000.

[48] L.R. Thebaud, "Systems and methods with identity verification by comparison and interpretation of skin patterns such as fingerprints," US Patent No. 5909501, June 1999.

[49]J. Feng, Z. Ouyang, and A. Cai, "Fingerprint matching using ridges," Pattern Recognition, vol. 39, no. 11, pp. 2131-2140, Nov. 2006.

[50] M. Hara and H. Toyama, "Method and apparatus for matching streaked pattern image," US Patent No. 7,295,688, Nov. 2007.

[51] N.K. Ratha, et al, "Robust fingerprint authentication using local structural similarity", Fifth IEEE Workshop Applications on Applications of Computer Vision, pp. 29-34, Dec. 2000.

[52] A.M. Bazen and S.H. Gerez, "Fingerprint matching by thin-plate spline modelling of elastic deformations", Pattern Recognition, vol. 36, no. 8, pp. 1859-1867, Aug. 2003.

[53]J. Feng, and A. K. Jain, "Fingerprint reconstruction: From minutiae to phase", IEEE Transactions on PAMI, vol. 33, no. 2, pp. 209 - 223, Feb. 2011.

[54] C. Hill, "Risk of masquerade arising from the storage of biometrics", Master's Thesis, Australian National University, 2001.

[55]A.Ross, J. Shah, and A.K.Jain, "From template to image: Reconstructing fingerprints from minutiae points", IEEE Trans. PAMI, vol. 29,no. 4, pp. 544-560, Apr. 2007. 
[56]B.G.Sherlock and D.M.Monro, "A model for interpreting fingerprint topology", Pattern Recognition, vol.26, no.7, pp.1047-1055, July 1993.

[57]R. Cappelli, et al, "Fingerprint image reconstruction from standard templates", IEEE Trans. PAMI, vol. 29, no. 9, pp. 1489-1503, Sep. 2007.

[58] P.R. Vizcaya and L.A. Gerhardt, "A nonlinear orientation model for global description of fingerprints," Pattern Recognition, vol. 29, no. 7, pp. 1221-1231, Jul. 1996.

[59] T-Y. Jea, "Minutiae-based partial fingerprint recognition", Master's Thesis, University at Buffalo, the State University of New York, Nov. 2005.

[60] S.O. Novikov and G.N.Glushchenko, "Fingerprint ridges structure generation models", Proc. SPIE Int'I Workshop Digital Image Processing and Computer Graphics, vol. 3346, pp. 270274, Oct. 1997.

[61]W. Bicz, "The idea of description (reconstruction) of fingerprints with mathematical algorithms and history of the development of this idea at Optel", Optel, http://www.optel.pl/article/english/ idea.htm, 2003.

[62] A.Witkin and M.Kass, "Reaction-Diffusion textures", ACM SIGGRAPH Computer Graphics, vol. 25, no. 4, pp. 299-308, July 1991.

[63] K.Nandakumar, A.K.Jain and S.Pankanti, "Fingerprint-based fuzzy vault: Implementation and performance," IEEE Transactions on Information Forensics and security, vol.2, no. 4, pp.744-757, Dec. 2007.

[64] K.A.Nixon and R.K.Rowe, "Multispectral fingerprint imaging for spoof detection", Biometric Technology for Human Identification II, A. K. Jain and N. K. Ratha, eds., vol. 5779, pp. 214225,SPIE, Mar. 2005

[65] K.G.Darpanis, “Gabor Filters”, York University, April 2007.

[66] A. Ullah, R. Khan and M. Shakeel, "Fingerprint recognition and password security system", Project, http://www.scribd.com/doc/17264412/fingerprint-and-password-security-systemthesis", July 2009 
[67]L. O'Gormann and J.V.Nickerson, "An approach to fingerprint filter design", Pattern Recognition, vol. 22, no. 1, pp.29-38, Jan. 1989.

[68] D. Maltoni et al, "Handbook of fingerprint recognition”, Springer, 2003.

[69] R. Cappelli, "Synthetic fingerprint generation", in D. Maltoni, D. Maio, A.K. Jain and S. Prabhakar, "Handbook of fingerprint recognition" 2nd Edition, Springer, London, 2009.

[70]M. C. Fairhurst, "Signature verification revisited: Promoting practical exploitation of biometric technology", Electronics \& Communication Engineering Journal, vol. 9, no. 6, pp. 273-280, Dec. 1997.

[71]H. Feng and C.Wa, "Private Key generation from on-line handwritten signatures", Information Management and Computer Security, vol. 10, no. 4, pp.159-164, Oct. 2002.

[72] S. K. Mitra and G. L .Sicuranza, "Nonlinear image processing", Academic Press, New York, 2000.

[73] G.Ramponi and G.L.Sicuranza, "Quadratic digital filters for image processing", IEEE Transactions on Acoustics, Speech and Signal processing, vol. 36, no. 6, pp. 937-939, June 1988.

[74] B.M. Mehtre and B. Chatterjee, "Segmentation of fingerprint images - a composite method", Pattern recognition, vol. 22, no. 4, pp. 381-385, 1989.

[75] A. Ross, J. Shah and A.K. Jain, "Towards reconstructing fingerprints from minutiae points", Proc. of SPIE conference on biometric technology for human identification II, vol. 5779, pp. 66-80, March 2005.

[76] L. Shapiro and G. Stockman, "Computer vision”, Prentice Hall, New Jersey, 2001.

[77] L.-M. Liu and T-S. Dai, "A hybrid fingerprint enhancement algorithm", International Conference on Computer Engineering and Systems, pp. 57-62, 2009.

[78] B.M. Mehtre, "Fingerprint image analysis for automatic identification", Machine Vision and Applications, vol. 6, no. 2-3, pp. 124-139, March 1993. 
[79]B. Moayer and K.S. Fu, "A syntactic approach to fingerprint pattern recognition", Pattern Recognition, vol. 7, no.1-2, pp. 1-23, June 1975.

[80] C.-T. Lu, K.-F. Tseng and C.-T. Chen, "Reduction of residual noise using directional median filter", IEEE International Conference on Computer Science and Automation Engineering (CSAE), vol. 3, pp. 475 - 479 , June 2011.

[81] SEIR Optics Technology whitepaper, "http://www.secugen.com/company/ip.htm"

[82] A. V. Oppenheim and R. W. Schafer, "Discrete-Time signal processing", Prentice Hall, 3rd Edition, Aug. 2009.

[83] R.C. Gonzalez and R.E.Woods, "Digital image processing", Prentice Hall, 3rd Edition, Aug. 2007.

[84] The Organization of the Retina and Visual System http://webvision.med.utah.edu/book/parti- foundations/gross-anatomy-of-the-ey

[85] Gait cycle http://www.orthoteers.com/\%28S\%28kfcgxubarh2xgq4vbmnwekp5\%29\%29/mainpage.aspx ?section $=23$

[86] L. Ji, et al, "Binary fingerprint image thinning using template-based PCNNs", IEEE Trans on Systems, Man and Cybernatics. Part B Cybernatics, vol. 37, no. 5, pp. 1407-1413, Oct 2007.

[87] D. J. Heeger, "Optical flow using spatiotemporal filters", International Journal of Computer Vision, vol. 1, no. 4, pp. 279-302, 1987. 


\section{BIOGRAPHICAL INFORMATION}

Sreya Chakraborty has completed her Masters in Electrical Engineering from University of Texas at Arlington,USA. She did her undergraduate from Mumbai University, India. Her research interest include Image Processing. She has received Graduate scholarship from the Electrical department for the year 2010-2011 and also has been the recipient of Korea scholarship for her work in the area at Masters level. She has also been the recipient of Ratan Tata scholarship at her undergraduate level. 